\title{
Cross-HLA targeting of intracellular oncoproteins with peptide-centric CARs
}

https://doi.org/10.1038/s41586-021-04061-6

Received: 20 April 2021

Accepted: 23 September 2021

Published online: 3 November 2021

\section{Open access}

Check for updates

\author{
Mark Yarmarkovich', Quinlen F. Marshall', John M. Warrington', Rasika Premaratne ${ }^{2}$, \\ Alvin Farrel ${ }^{1,3}$, David Groff', Wei Li ${ }^{4}$, Moreno di Marco ${ }^{5}$, Erin Runbeck', Hau Truong', \\ Jugmohit S. Toor ${ }^{6}$, Sarvind Tripathi ${ }^{6}$, Son Nguyen ${ }^{7}$, Helena Shen', Tiffany Noel', \\ Nicole L. Church ${ }^{2}$, Amber Weiner', Nathan Kendsersky', Dan Martinez ${ }^{8}$, Rebecca Weisberg', \\ Molly Christie', Laurence Eisenlohr ${ }^{8}$, Kristopher R. Bosse ${ }^{1,9}$, Dimiter S. Dimitrov ${ }^{4}$, \\ Stefan Stevanovic ${ }^{5}$, Nikolaos G. Sgourakis', Ben R. Kiefel ${ }^{2}$ \& John M. Maris ${ }^{1,9 凶}$
}

The majority of oncogenic drivers are intracellular proteins, thus constraining their immunotherapeutic targeting to mutated peptides (neoantigens) presented by individual human leukocyte antigen (HLA) allotypes ${ }^{1}$. However, most cancers have a modest mutational burden that is insufficient to generate responses using neoantigen-based therapies ${ }^{2,3}$. Neuroblastoma is a paediatric cancer that harbours few mutations and is instead driven by epigenetically deregulated transcriptional networks ${ }^{4}$. Here we show that the neuroblastoma immunopeptidome is enriched with peptides derived from proteins that are essential for tumourigenesis and focus on targeting the unmutated peptide QYNPIRTTF, discovered on HLA-A*24:02, which is derived from the neuroblastoma dependency gene and master transcriptional regulator $P H O X 2 B$. To target QYNPIRTTF, we developed peptide-centric chimeric antigen receptors (CARs) using a counter-panning strategy with predicted potentially cross-reactive peptides. We further hypothesized that peptide-centric CARs could recognize peptides on additional HLA allotypes when presented in a similar manner. Informed by computational modelling, we showed that PHOX2B peptide-centric CARs also recognize QYNPIRTTF presented by HLA-A*23:01 and the highly divergent HLA-B*14:02. Finally, we demonstrated potent and specific killing of neuroblastoma cells expressing these HLAs in vitro and complete tumour regression in mice. These data suggest that peptide-centric CARs have the potential to vastly expand the pool of immunotherapeutic targets to include non-immunogenic intracellular oncoproteins and widen the population of patients who would benefit from such therapy by breaking conventional HLA restriction.
The curative potential of CAR T cell-based cancer immunotherapies has been established in leukaemias, but their application in solid tumours has been limited by a paucity of known tumour-specific membrane proteins ${ }^{5}$. Although membrane proteins represent up to a quarter of the proteome, only a fraction of these are expressed specifically on tumour cells and not on normal tissues, and a smaller proportion are essential to tumour homeostasis ${ }^{6}$. Rather, most cancer driver proteins reside in the cytoplasm or nucleus of the cell, where they are accessible to the immune system only through presentation of peptides on the major histocompatibility complex (MHC).

MHC class I proteins, encoded by the highly polymorphic human leukocyte antigen (HLA)-A, -B and -C genes, present a snapshot of the intracellular proteome on the cell surface (immunopeptidome) where T cells surveil the peptide-MHC complexes (pMHC) for antigens derived from foreign pathogens. T cell recognition of mutation-derived pMHC neoantigens as non-self is the basis of curative responses achieved through immune checkpoint blockade and complete remissions using adoptive transfer of tumour-infiltrating lymphocytes (TILs) ${ }^{7}$. Nonetheless, only about $5 \%$ of these neoantigens are predicted to bind a given HLA allotype ${ }^{8}$, and just $1.6 \%$ of neoantigens are reported to be immunogenic ${ }^{3}$. Subclonal mutations and downregulation of mutated non-essential genes further constrain the pool of therapeutically relevant neoantigens, necessitating a mutational threshold for effective neoantigen-based therapies that is not surpassed in most cancers ${ }^{9}$. Tumour cells also present a wide range of unmutated self-peptides on $\mathrm{MHC}^{10}$ molecules, but these are largely immunogenically silent owing to negative thymic selection of T cells. We hypothesized that a subset of the immunopeptidome consists of tumour-specific peptides derived from essential oncoproteins and that these can be targeted using synthetic peptide-centric CARs (PC-CARs).

${ }^{1}$ Division of Oncology and Center for Childhood Cancer Research, Children's Hospital of Philadelphia, Philadelphia, PA, USA. ${ }^{2}$ Myrio Tx, Melbourne, Victoria, Australia. ${ }^{3}$ Department of Biomedical and Health Informatics, Children's Hospital of Philadelphia, Philadelphia, PA, USA. ${ }^{4}$ University of Pittsburgh, Pittsburgh, PA, USA. ${ }^{5}$ University of Tubingen, Tubingen, Germany.

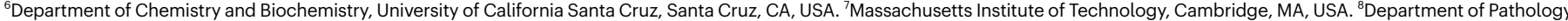

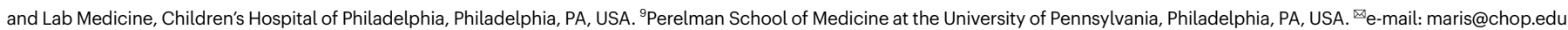


Peptides presented in the MHC groove make up only a small fraction of the extracellular pMHC molecular surface. The typical 8-14mer peptide presented on MHC class I comprises only around 2-3\% of the amino acids in the pMHC complex and is spatially confined within the adjacent $\alpha$-helices of the MHC groove, thus posing major challenges for engineering peptide-specific single-chain antibody variable fragment (scFv) binders ${ }^{11}$. Furthermore, cross-reactivity of engineered receptors with peptides of biophysically similar molecular surfaces presented in normal tissues have resulted in significant toxicity and death ${ }^{12,13}$.

Neuroblastoma is a childhood cancer derived from tissue of the developing sympathetic nervous system and is often lethal despite intensive cytotoxic therapy ${ }^{14}$. These tumours are low in mutational burden ${ }^{15}$ and MHC expression ${ }^{16}$, making neuroblastoma both a challenging tumour to target with MHC-based immunotherapies and an ideal model for addressing the major problems currently hindering the wider advancement of cancer immunotherapies. As a tumour derived from neural crest progenitor cells, neuroblastomas express a set of core-regulatory circuit (CRC) transcription factors involved in maintaining cell fate, metabolism, migration, epigenetic states, growth and proliferation ${ }^{4}$. These genes are epigenetically silenced upon terminal differentiation of normal neural tissues, but these developmental pathways are aberrantly hyperactivated in neuroblastoma. Here we present the discovery of recurring lineage-restricted oncoproteins presented on $\mathrm{MHC}$, focusing on immunotherapeutic targeting of the neuroblastoma $\mathrm{CRC}$ master regulator $\mathrm{PHOX} 2 \mathrm{~B}$ using PC-CARs.

\section{Identification of tumour-specific antigens}

First, we surveyed the landscape of peptides accessible to T cells by performing MHC capture, peptide elution and liquid chromatography with tandem mass spectrometry ${ }^{17}$ (immunopeptidomics) on eight neuroblastoma cell-derived xenografts (CDX) and patient-derived xenografts (PDX) showing a wide range of MHC expression and also encompassing the array of rare recurring mutations found in high-risk neuroblastoma ${ }^{18,19}$ (Fig. 1a, Extended Data Table 1a). We identified a total of 7,608 peptides from 8 tumours ( $1 \%$ false discovery rate (FDR); Supplementary Table 1), finding none of the 4,105 potential 8-14mer neoantigens imputed from tumour mutational data in the immunopeptidome, consistent with expected rates of neoantigen presentation and limited detection using ligandomics in low-mutational tumours $^{20}$. We first filtered the 7,608 peptides to select HLA binders with sufficient affinity to act as $\mathrm{T}$ cell epitopes using a predicted peptide-MHC (pMHC) half-maximal inhibitory concentration $\left(\mathrm{IC}_{50}\right)$ threshold of $500 \mathrm{nM}$, yielding 2,286 predicted strong binders. We then filtered for peptides derived from differentially expressed parent genes as determined from RNA-sequencing (RNA-seq) data from 153 neuroblastoma tumours compared with 1,641 normal tissues (from the TARGET ${ }^{21}$ and GTEx ${ }^{17}$ databases, respectively), resulting in 61 peptides derived from genes with mRNA expression one log-fold higher in tumour for each normal tissue $(P<0.01)$. Finally, we filtered the remaining tumour peptides against a database of MHC peptides empirically characterized on 190 normal tissues ${ }^{22}$, removing any peptide with a parent gene that is represented in the normal tissue immunopeptidome. While this last step does not absolutely exclude potential presentation of a peptide in normal tissues, it allowed us to narrow our list to 13 peptides derived from 9 unique genes expressed in neuroblastoma that have not been previously detected in any normal tissue.

We then performed immunopeptidomics on eight high-risk diagnostic neuroblastoma primary tumours, focusing on HLA-A*02:01 and HLA-A*24:02 allotypes (Extended Data Table 1a, Supplementary Table 1). Using the same filtering as described for xenograft tumours, we identified 56 peptides ( 33 unique peptides derived from 29 unique proteins) with strong HLA-binding affinity derived from differentially expressed genes in neuroblastoma and not previously detected in the benign tissue ligandome (Fig. 1b, Extended Data Fig. 1). We confirmed the presence of 7 of the 13 peptides from the xenografts in primary tumours, suggesting that CDX and PDX tumours can be a predictive model for primary tumour ligandomes. Notably, the most enriched gene ontologies of the peptide parent genes not previously observed in the normal-tissue ligandomic dataset were: noradrenergic neuron differentiation $\left(P=3.42 \times 10^{-4}, \mathrm{FDR}=0.0389\right)$ and sympathetic nervous system development $\left(P=2.95 \times 10^{-5}, \mathrm{FDR}=0.00665\right.$; Fig. $1 \mathrm{c}$, green bar). These findings highlight the distinctiveness of the neuroblastoma ligandome and suggest that MHC-presented peptides are enriched for those derived from aberrantly expressed lineage-restricted genes.

To select those peptides with the highest potential as putative immunotherapeutic targets, we prioritized peptides on the basis of pMHC binding affinity, HLA allele frequency, degree of differential expression of parent genes, relative abundance on MHC as compared to other peptides, recurrence across multiple neuroblastoma tumours and relevance to neuroblastoma biology based on the published literature ${ }^{23-25}$. One peptide each from CHRNA3, GFRA2, HMX1, IGFBPL1, PHOX2B and TH were selected for preclinical development (Fig. 1d, e; Supplementary Table 1). The presence of these peptides in tumours was validated by performing liquid chromatography-mass spectrometry (LC-MS/MS) on synthetic peptides (Extended Data Fig. 2), yielding complete concordance across $b$ and $y$ ions. In addition, we validated the peptide binding to predicted HLA alleles by refolding the PMHC complex and solving the crystal structure of one $\mathrm{PHOX} 2 \mathrm{~B}$ peptideHLA-A*24:02 (PDB ID 7MJA) and three IGFBPL1 peptide-HLA-A*02:01 (PDB IDs 7MJ6, 7MJ67 and 7MJ8) protein complexes (Fig. 1f, Extended Data Fig. 3b). Of note, we detected three unique IGFBPL1 peptides as a $9 \mathrm{mer}, 11 \mathrm{mer}$ and $12 \mathrm{mer}$ (all with the same core 9 mer amino acids) with distinct thermal properties (Extended Data Fig. 3), underscoring the existence of multiple peptides with distinct conformations arising from the same region of a protein and presenting multiple opportunities for therapeutic targeting ${ }^{26}$.

We then inferred the ability of a tumour to evade the immune response via downregulation of target genes by examining the binding of neuroblastoma CRC transcription factors (MYCN, ASCL1, HAND2, ISL1, PHOX2B, GATA3 and TBX2; Extended Data Fig. 4a) to the parent gene locus of the prioritized antigens ${ }^{4}$. We found that all six CRC proteins bind regulatory elements at each parent gene locus within H3K27Ac super-enhancer elements (Fig. 1g, Extended Data Fig. 4b), suggesting that transcriptional redundancy and dependency should mitigate the risk of antigen loss in response to immunotherapy owing to downregulation of the parent gene. In addition, we found peptides from each of the six CRC proteins represented in the neuroblastoma immunopeptidome (Extended Data Table 1b). Indeed, the most significantly enriched gene groups in the immunopeptidome are nucleic acid-binding proteins $\left(P=9.5 \times 10^{-17}\right.$; Extended Data Fig. $\left.5 \mathrm{c}\right)$. Finally, we interrogated the dynamics of gene expression during development using temporal transcriptomic data ${ }^{27}$. Consistent with its function in orchestrating neural crest progenitor development ${ }^{28}$, PHOX2B is expressed exclusively during fetal development and is completely silenced in normal tissues before birth (Fig. 1h), as are IGFBPL1, TH and CHRNA3 (Extended Data Fig. 6). PHOX2B is a key CRC protein that is among the most specifically expressed genes in neuroblastoma ${ }^{29}$. PHOX2B expression is not detected in normal tissue, unlike many solid tumour immunotherapy targets, including HER2, and chemotherapy targets in neuroblastoma camptothecin, including TOP1, each of which exhibit significant normal tissue expression (Fig. 1e). PHOX2B expression is routinely used in neuroblastoma diagnostic assays ${ }^{30}$, is one of two highly penetrant neuroblastoma susceptibility genes ${ }^{31}$, and is the third most significant dependency in neuroblastoma, as reported in DepMap ${ }^{32}$. Together, these data suggest that PHOX2B is a highly specific tumour antigen in neuroblastoma and an ideal candidate for therapeutic targeting. 


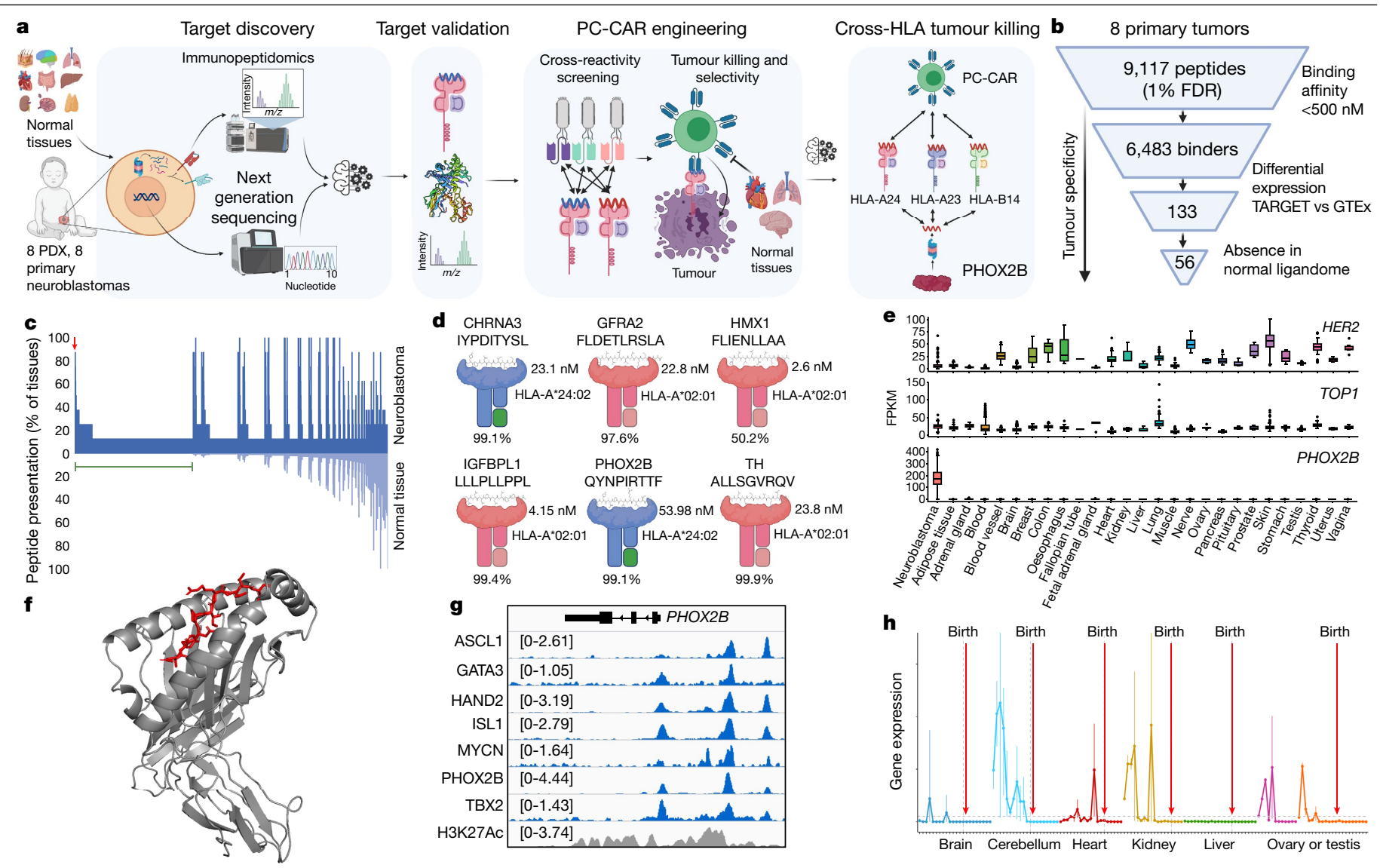

Fig. 1 An antigen discovery and prioritization process identifies PHOX2B as a target for immunotherapy. a, Summary of tumour-antigen discovery and CAR-engineering workflow: (1) integrated genomics and immunopeptidomics process; (2) target validation; (3) CAR engineering; and (4) cross-HLA tumour killing. b. Computational filtering of 9,117 peptide instances identified by immunopeptidomics in primary tumours ( $1 \% \mathrm{FDR}$ ) resulted in 56 neuroblastomaspecific peptides (33 unique peptides) derived from 29 distinct proteins. c, Primary neuroblastoma tumour immunopeptidome compared with 190 normal tissues. Each point on the $x$-axis represents one of 5,832 unique peptides identified in primary tumours, with the proportion of neuroblastoma tumours presenting a given peptide annotated above axis in dark blue and the proportion of normal tissue expressing the identical peptide below the axis in light blue. The green horizontal bar indicates 1,492 peptides not previously observed in the normal tissue immunopeptidome. Parent genes from neuroblastomaspecific peptides resulted in the top two GO enrichment terms: noradrenergic neuron differentiation and sympathetic nervous system development.
The arrow denotes 351 recurring peptides presented in neuroblastoma that were not previously detected in normal tissues. d, Five antigens differentially expressed in PDX and primary tumours and further prioritized for analysis, HLA allele frequency, relative peptide abundance (percentile rank annotated below $\mathrm{pMHC}$ ), predicted pMHC-binding affinity, and relevance to neuroblastoma tumourigenesis.e, $P H O X 2 B$ expression in RNA-seq data from 153 neuroblastoma tumours versus 1,641 normal tissues in GTEx. PHOX2B expression is restricted to tumours, compared with the immunotherapy target HER2 and the neuroblastoma chemotherapy target TOP1 (note differences in the $y$-axis scales). FPKM, fragments per kilobase of transcript per million mapped reads.f, Crystal structure of PHOX2B 9 mer QYNPIRTTF (red) refolded with HLA-A*24:02 (grey). g, Chromatin immunoprecipitation followed by DNA sequencing (ChIP-seq) of neuroblastoma tissue shows binding of all CRC proteins at the $P H O X 2 B$ locus and association with a $\mathrm{H} 3 \mathrm{~K} 27 \mathrm{Ac}$ super-enhancer mark. h, RNA-seq of fetal tissue shows that $P H O X 2 B$ is expressed in early development and downregulated before birth across seven tissues. Created with BioRender.com.
Before developing an immunotherapeutic construct targeting the PHOX2B peptide, we validated that low MHC expression in neuroblastoma ${ }^{16}$ did not prohibit $\mathrm{T}$ cell engagement and activation using an influenza antigen model (experimental details in Extended Data Fig. 7).

\section{PC-CAR T cell engineering for $P H O X 2 B$}

Owing to the low immunogenicity of self-antigens, we pursued development of scFv-based CARs rather than engineered $\mathrm{T}$ cell receptors (TCRs) for PHOX2B after no high affinity TCRs were identified in multiple screens (Extended Data Fig. 8). We reasoned that immunogenicity could be induced to otherwise immunogenically inert pMHCs using synthetic, peptide-centric receptors. Our first generation of pMHC-directed CARs showed significant cross-reactivity to the MHC, which we were able to abrogate using saturation mutagenesis techniques that also decreased binding affinity (Extended Data Figs. 9-11).
To screen for PHOX2B peptide-specific clones, we next adapted the retained display ${ }^{33}(\mathrm{ReD})$ system, a protein-display platform that enables the flow-cytometric selection of pMHC-binding scFvs in permeabilized bacterial cells, with a scFv library containing more than $10^{11}$ variants. Clones that demonstrated apparent selectivity by flow cytometry were further tested against a panel of 95 unrelated peptides and four highly similar peptides presented on the same HLA allotype to select for clones with the highest selectivity (for example, selective clone 10LH, shown in Fig. 2a), resulting in $25 \mathrm{scFv}$ binders for screening in CAR T constructs. To address cross-reactivity with pMHC in normal tissues, we developed an algorithm to predict potential selective cross-reactive antigen presentation (sCRAP; https://marisshiny.research.chop.edu/ sCRAP/) on the same HLA allotype (Extended Data Fig. 12), enabling pre-emptive selectivity filtering in early stages of $\mathrm{scFv}$ screening without the need of a prior receptor or alanine $\operatorname{scan}^{34}$. We benchmarked the sCRAP algorithm by testing its ability to predict the cross-reactivity of 

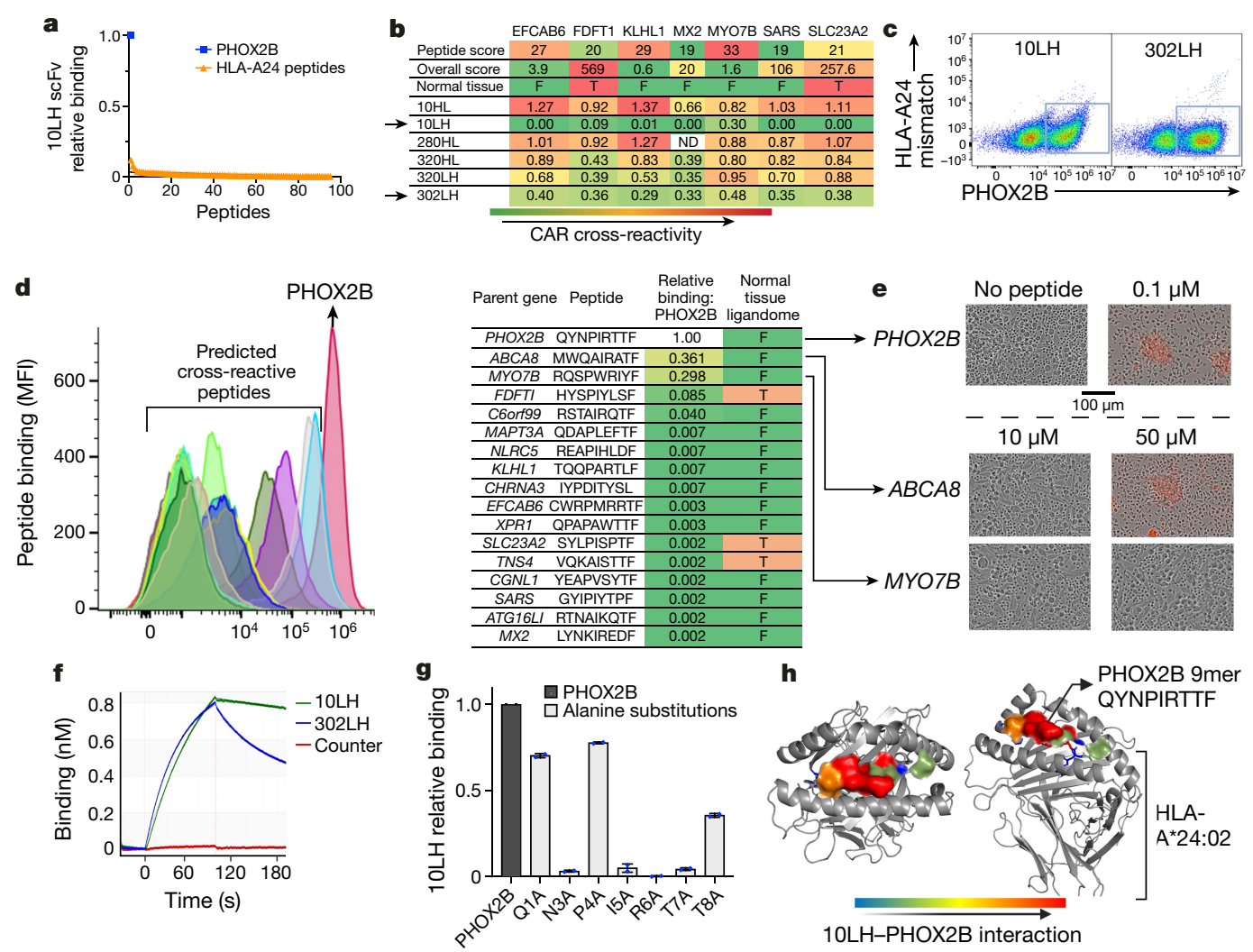

Fig. 2 | Engineering pMHC-specific CAR receptors. a, Ranked binding affinity of 10LH scFv to PHOX2B (blue) and a panel of 95 peptides presented on HLA-A*24:02 peptides (orange) demonstrate high target binding and negligible binding to HLA-A*24:02 pMHCs. b, Cross-reactivity algorithm identifies CAR constructs with significant off-target binding and informs prioritization of highly selective receptors (selective receptors marked with arrow). Peptide score represents the predicted cross-reactivity based on the amino acid sequences of normal-tissue peptides; overall score is calculated on the basis of peptide score, binding affinity and normal tissue expression; T, peptides reported in the normal tissue immunopeptidome; $F$, peptides absent in normal immunopeptidome. ND, not determined.c, Example counterstaining of top CAR clones with target ( $x$-axis) and off-target ( $y$-axis) peptides on HLA-A*24:02 reveals selective target binding in $10 \mathrm{LH}$ and $302 \mathrm{LH}$ constructs. $d$, Left, flow cytometry plot of predicted cross-reactive peptides compared with PHOX2B shows cross-reactive binders ABCA8 and MYO7B. Right, flow mean fluorescence intensity (MFI) used to calculate degree of binding relative to $\mathrm{PHOX} 2 \mathrm{~B}$ in table. e, Functional screening of ABCA8 and MYO7B shows CAR killing through $\mathrm{ABCA} 8$ only at a supraphysiological concentration of $50 \mu \mathrm{M}$ versus through PHOX2B at $0.1 \mu \mathrm{M}$. ABCA8 and MYO7B were not detected in the normal tissue immunopeptidome, and none of the peptides predicted by sCRAP that were detected in the normal immunopeptidome (FDFTI, SLC23A2 and TNS4) show binding to 10LH. The experiment was performed once on the entire panel of CAR constructs and repeated for $10 \mathrm{LH}$ and $302 \mathrm{LH}$ on an expanded panel of peptides.f, Representative BLItz plot at $200 \mathrm{nM}$ PHOX2B pMHC shows fast on rate for $10 \mathrm{LH}$ and $302 \mathrm{LH}$ and exceptionally slow off-rate for $10 \mathrm{LH}$

$\left(k_{\mathrm{d}}=7.6 \times 10^{-4} \mathrm{~s}^{-1}\right) . \mathrm{g}$, Alanine scan of QYNPIRTTF reveals that mutations in five residues (N3A, I5A, R6A, T7A and T8A) result in significant abrogation of binding to PC-CAR 10LH ( $n=2$; data are mean \pm s.d.).h, PHOX2B-HLA-A*24:02 crystal structure paired with alanine scan of $10 \mathrm{LH}$ enables mapping of peptidereceptor interface, revealing spatial conformation of five receptor contact residues (left, top view; right, side view of pMHC complex). Created with BioRender.com. the MAGE-A3 peptide presented on $\mathrm{HLA}_{-} \mathrm{A}^{*} 01: 01$, whose targeting using an affinity-enhanced TCR previously resulted in the fatal cross-reaction with another peptide derived from the TITIN protein presented on HLA-A ${ }^{*} 01: 01$ in myocardial tissues ${ }^{12}$. We predicted the cross-reactivity of MAGE-A 3 with the TITIN peptide as the 4 th-ranked prediction out of $1,143,861$ potential self-peptides presented in heart tissue (Extended Data Fig. 13).

We then screened our panel of PHOX2B-directed CARs against the top seven pMHC predicted by SCRAP (Fig. 2b), allowing us to eliminate cross-reactive CARs and prioritize those with the highest degree of target selectivity (Fig. 2c). Of 25 CARs screened, we selected clone $10 \mathrm{LH}-$ which had the highest-specificity profile, showing only two peptides with more than $10 \%$ relative binding to $10 \mathrm{LH}$ as compared to PHOX2B-for further development (Fig. 2d).

To test the functional significance of the binding to potential off-target pMHCs predicted by SCRAP, we pulsed HLA-matched PHOX $2 \mathrm{~B}^{-}$SW620 colon adenocarcinoma cells with the $\mathrm{PHOX} 2 \mathrm{~B}$ peptide and potential cross-reactive peptides across a range of concentrations.
Pulsing with the PHOX2B peptide resulted in complete cytotoxicity when co-cultured with $10 \mathrm{LH}$ at the lowest tested concentration of $0.1 \mu \mathrm{M}$. The 10LHCAR T cells did not induce cytotoxicity with the most cross-reactive predicted peptide $\mathrm{ABCA} 8$ at $10 \mu \mathrm{M}$, and only induced killing at the supraphysiological concentration of $50 \mu \mathrm{M}$. The second most cross-reactive peptide with 10LH (MYO7B) showed no CAR cytotoxicity at concentrations of up to $50 \mu \mathrm{M}$ (Fig. 2e). Neither ABCA8 nor MYO7B has been detected in normal tissue immunopeptidome $\mathrm{e}^{10}$, and none of the peptides previously detected in the normal tissue immunopeptidome display any cross-reactivity with PC-CAR 10LH (Fig. 2d). These screens demonstrate the utility of sCRAP for pre-emptively identifying off-target effects, efficiently screening their functional consequences and identifying binders with highly selective binding to tumour targets.

The lead scFv 10LH bound PHOX2B pMHC with a dissociation constant $K_{\mathrm{D}}$ of $13 \mathrm{nM}$ and exceptionally slow off rate $\left(k_{\mathrm{d}}\right)$ of $7.6 \times 10-4 \mathrm{~s}^{-1}$ (Fig. 2f). We next performed an alanine scan for the 10 LH CAR, characterizing binding to $\mathrm{PHOX} 2 \mathrm{~B}$ pMHC with amino acid substitutions at each non-anchor position of the peptide ${ }^{34}$. The alanine scan revealed 

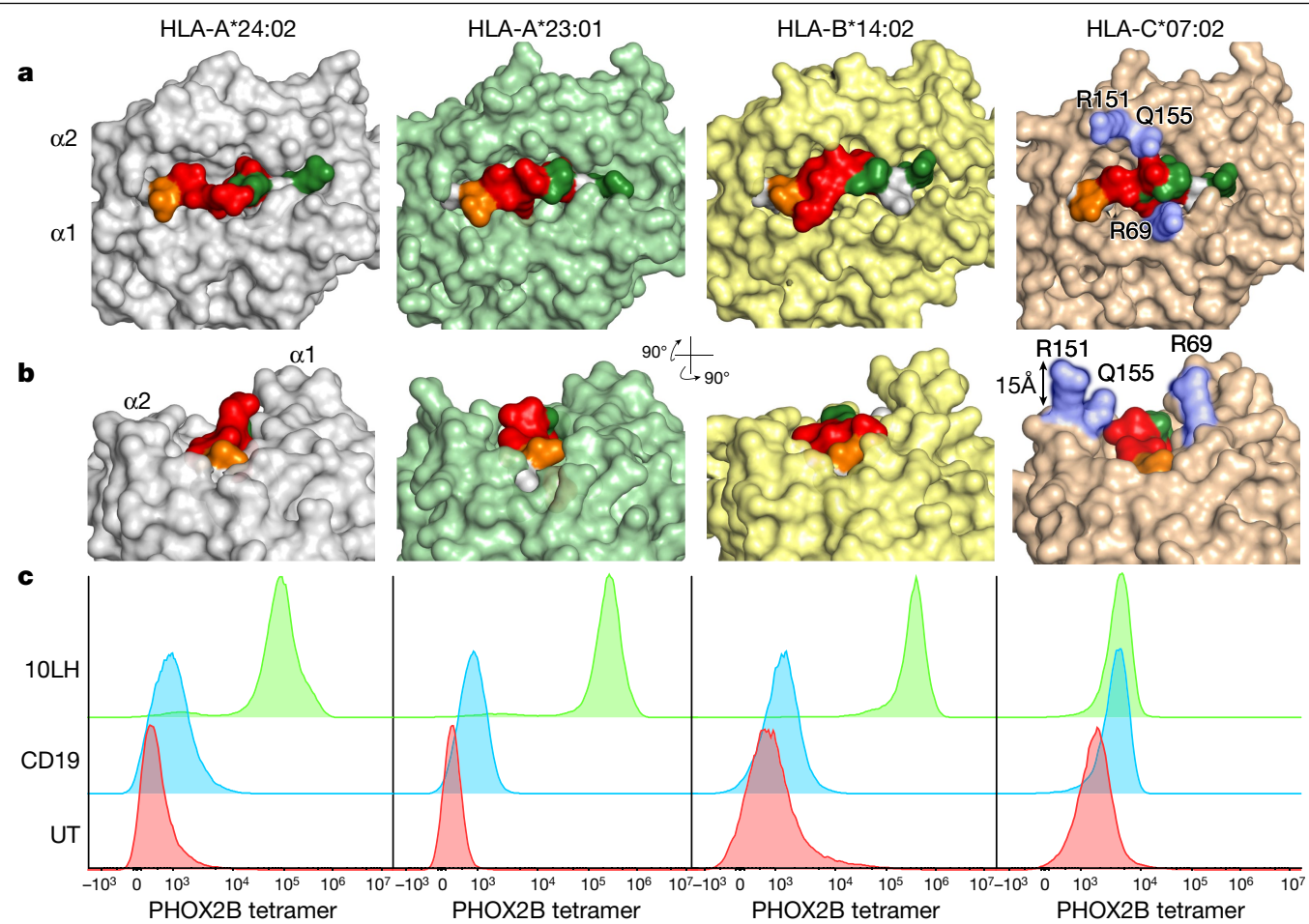

Fig. 3 | Structural basis of CARs binding to PHOX2B peptide presented on multiple HLAs. a, PHOX2B-HLA-A*24:02 crystal structure and models of PHOX2B in complex with HLA-A*23:01, HLA-B*14:02 and HLA-C*07:02. b. The charged and polar R151, Q155 and R69 residues of HLA-C* $07: 02$ align with key $10 \mathrm{LH}$ interaction residues I5, R6 and 17 (MHC residues in blue and
PHOX2B-10LH interaction residues in red). R151, Q155 and R69 create steric and charged hindrance of key peptide-binding residues.c, Staining of the PHOX2B PC-CAR 10LH reveals strong binding to HLA-A*24:02, HLA-A*23:01 and HLA-B*14:02, but not to HLA-C*07:02. CD19, CD19-directed CAR; 10LH, PHOX2B PC-CAR; UT, untransduced T cells. Created with BioRender.com. significant interactions of the PC-CAR receptor with five out of seven non-anchor residues of the $\mathrm{PHOX} 2 \mathrm{~B}$ peptide, including key residues protruding from the MHC cleft (interaction interface of $10 \mathrm{LH}$ with PHOX2B pMHC mapped on crystal structures in Fig. 2h), highlighting the superior selectivity of PC-CARs compared with the three or four residues that typically interact with the $T C R^{35}$.

\section{PC-CAR T cells break HLA restriction}

Given the prerequisite of antigen processing and presentation necessary for detection of a given MHC peptide by immunopeptidomics, we hypothesized that identical peptides could be presented on additional HLA allotypes capable of binding a peptide's anchor resides, and that some of these peptides could be presented in a similar enough conformation to be recognized by peptide-centric scFv binders (Extended Data Fig. 14a). We tested this hypothesis using PHOX2B-specific CARs engineered to bind the PHOX2B 9mer presented on HLA-A*24:02 in a peptide-centric fashion. We used our population-scale antigen presentation tool ShinyNAP ${ }^{8}$ to identify additional HLA allotypes that could present the same PHOX2B peptide, identifying 8 additional HLAs predicted to bind the PHOX2B 9mer (Extended Data Fig.14b). We then used our pMHC structural modelling software, RosettaMHC ${ }^{36}$, to model the $3 \mathrm{D}$ conformation and binding free energy of peptides presented by additional HLA alleles, identifying HLA-A*23:01 and HLA-B*14:02 as top-scoring candidates for recognition by PC-CARs of the PHOX2B peptide originally discovered on HLA-A*24:02 (Fig. 3a, b). After validating binding of QYNPIRTTF to these alternate allotypes (Extended Data Fig. 14c), we measured the ability of $10 \mathrm{LH}$ to recognize these pMHCs, finding that in addition to HLA-A*24:02,10LH binds with high affinity to the PHOX2B 9mer QYNPIRTTF presented by HLA-A*23:01 and HLA-B*14:02 (Fig. 3c). We also found that although QYNPIRTTF binds to HLA-C*07:02, 10LH exhibited 17.4-fold lower binding to HLA-C*07:02, probably owing to a distinct pMHC molecular surface in which the sidechains of the MHC $\alpha 2$-helix (R151 and Q155) protrude by $15 \AA ̊$ at the position axially aligned with the key $10 \mathrm{LH}$ binding residues of QYNPIRTTF (I5 and R6) (Fig.3b). To demonstrate functionally relevant recognition of our prediction of PHOX2B presentation on HLA-A*23:01, we pulsed the HLA-A*23:01 PHOX2B ${ }^{-}$melanoma cell line WM873 with the QYNPIRTTF peptide, showing induction of antigen-specific killing in peptide-pulsed cells and no cytotoxicity in cells pulsed with mismatched peptide (Extended Data Fig. 14d). HLA-A*23:01 is the most common non-A2 allele in people with African ancestry, highlighting the potential of PC-CARs to expand clinical application to underserved populations. Finally, we reanalysed our immunopeptidomics data, performing a matched peptide analysis to identify lower-confidence potential peptide matches to QYNPIRTTF in additional samples in which the peak was not fragmented. We identified $\mathrm{m} / \mathrm{z}$ matches within 1 min of retention time across 6 out of 8 PDX lines and 7 out of 8 patient samples, each expressing one of the HLA alleles predicted by our analyses, suggesting that this peptide is ubiquitously expressed in neuroblastoma (Extended Data Fig. 14f). These findings warrant additional investigation into cross-HLA-targeting tumour self-antigens as well as neoantigens and demonstrate the potential to significantly expand the eligible patient population receiving peptide-centric scFv-based immunotherapies.

\section{PC-CAR T cells selectively eliminate tumours}

We next tested the on-target killing potential of $10 \mathrm{LH}$ using available HLA-A*24:02 and HLA-A*23:01 neuroblastoma cell lines (SKNAS, NBSD and SKNFI) and demonstrated complete tumour cell killing and potent IFN- $\gamma$ release after $24 \mathrm{~h}$ at 5:1 effector:target ratio (Fig. $4 a-c$, Supplementary Video 1). We tested the functional cross-reactivity of PC-CARs against the peptides presented by off-target tissues; PC-CARs showed no activity in three HLA-A*24:02 cell lines that do not express PHOX2B 

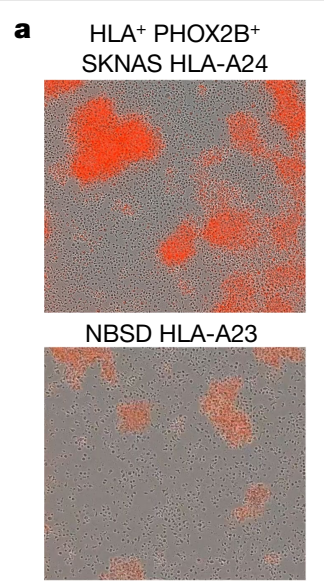

Cleaved caspase $3 / 7$
$\mathrm{HLA}^{+} \mathrm{PHOX}^{2} \mathrm{~B}^{-}$

SW620 HLA-A24

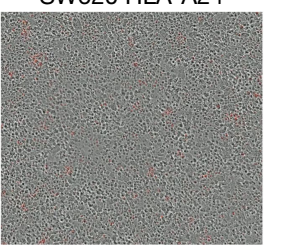

KATO III HLA-A24

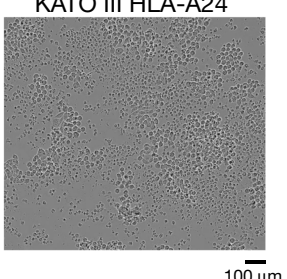

b
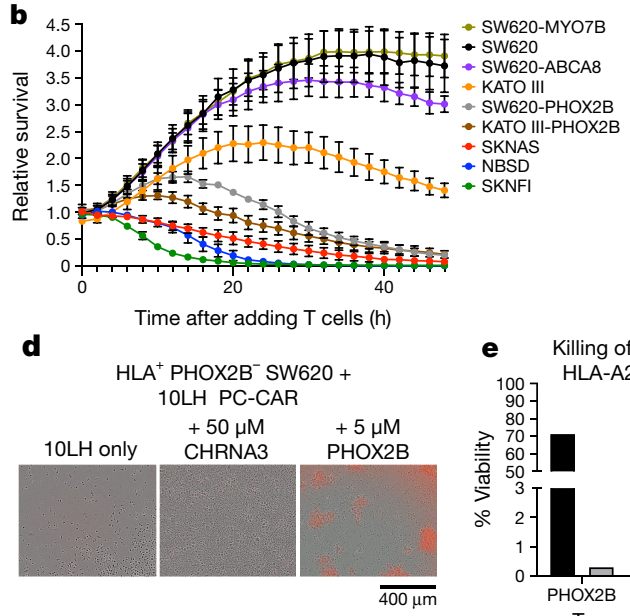

e

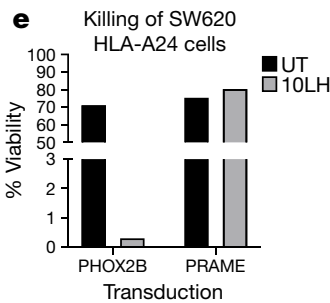

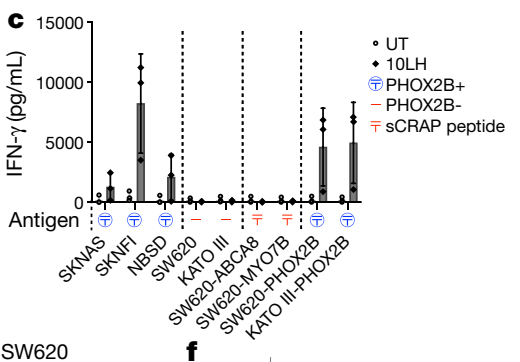

$\mathbf{f}$

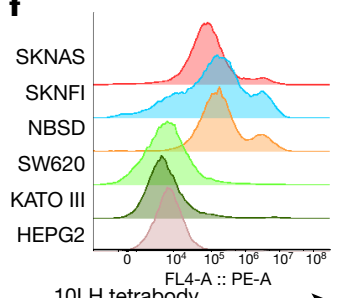

g
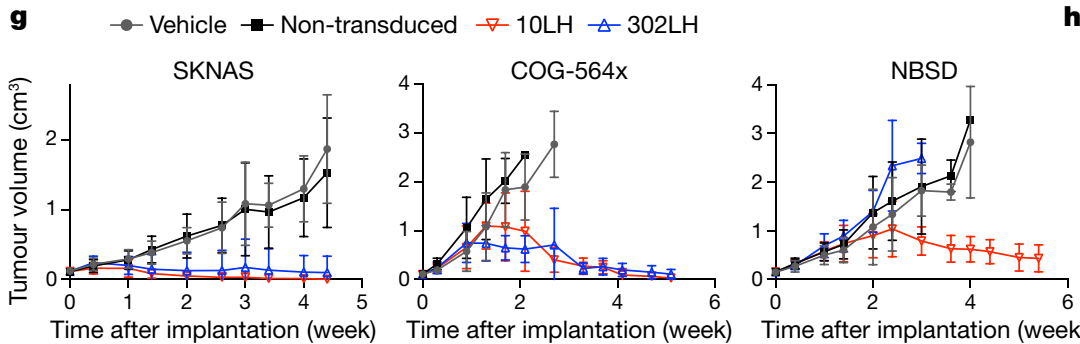

Time after implantation (week) h

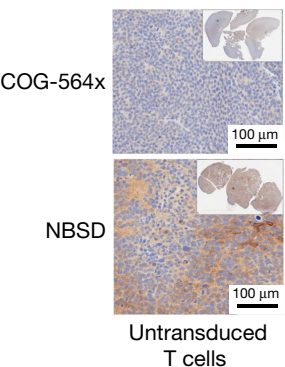

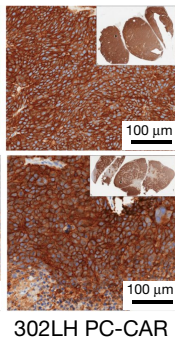

Fig. 4 | PHOX2B-specific PC-CAR T cells induce potent tumour killing in vitro and in vivo and break conventional $H L A$ restriction. $a-c$, The CAR $10 \mathrm{LH}$ induces specific killing and IFN- $\gamma$ release in neuroblastoma cells expressing HLA-A*24:02 and HLA-A*23:01 and PHOX2B (SKNAS, NBSD and SKNFI), but not in $\mathrm{HLA}_{-} \mathrm{A}^{*} 24: 02 \mathrm{PHOX}^{2} \mathrm{~B}^{-}$non-neuroblastoma tumour cells (SW620, HEPG2 and KATO III), unless PHOX2B peptide is added. No T cell activity was observed in SW620 when pulsed with $10 \mu \mathrm{M}$ of the predicted cross-reactive peptides ABCA8 or MYO7B (b,c). Cytotoxicity was visualized by T cell clustering and cleaved caspase (a), relative loss of confluence measured by loss of green fluorescence in GFP-transduced cancer cells in (b), and IFN- $\gamma$ release measured by ELISA (c). UT denotes untransduced T cells. Assays performed using T cells from $n=3$ donors, each in triplicate; data are mean \pm s.d. d, Pulsing HLA-A*24:02 PHOX2B cell line SW620 with $5 \mu \mathrm{M}$ PHOX2B induces complete cell killing when co-cultured with 10LHCAR, but no killing when pulsed with $50 \mu \mathrm{M}$ CHRNA3. Repeated across 3 experiments. e, 10LH CAR specifically and specifically kills SW620

(SW620 colorectal adenocarcinoma, KATO III gastric adenocarcinoma and HEPG2 hepatocellular carcinoma) (Fig. 4a-c, Extended Data Fig. 10b; Supplementary Video 2). To validate the specificity of killing mediated by PC-CARs, we pulsed HLA-matched, PHOX2B-negative cancer cell lines with the $\mathrm{PHOX} 2 \mathrm{~B}$ peptide as well as forcibly over-expressing PHOX2B. We demonstrated specific killing only in cells pulsed with PHOX2B peptide and those transduced with full length $P H O X 2 B$ mRNA, and not in cells pulsed with non-specific CHRNA3, ABCA8 and MYO7B peptides presented on the same HLA, nor in cells transduced with full length PRAME mRNA (Fig. 4d, e), demonstrating that native PHOX2B is processed and presented on $\mathrm{MHC}$, where it is specifically recognized by PC-CARs. To detect PHOX2B pMHC on the cell surface, we generated a tetramerized $10 \mathrm{LH}$ scFv and stained on-target and off-target cell lines, which showed significant surface PHOX2B pMHC in neuroblastoma cells and not in HLA-matched controls (Fig. 4f, Extended Data Fig. 15a), suggesting that these reagents have the potential to be used to assess the presence of antigen in biopsied tissue samples. We also found that CARs flagged as cross-reactive by sCRAP demonstrated significant cross-reactivity, validating the functional consequences of cross-reactivities predicted by our algorithm (Extended Data Fig. 15b). control cells transduced with PHOX2B, but not with PRAME. f, Staining cancer cells with tetramerized $10 \mathrm{LH}$ scFv enables detection of $\mathrm{PHOX} 2 \mathrm{~B}$ pMHC on neuroblastoma cells but not in HLA-matched controls. g, PHOX2B-specific PC-CAR T cells induce potent tumour killing in mice engrafted with neuroblastoma PDX tumours, including the extremely fast-growing line COG-564x and HLA-A*23:01 line NBSD. $n=6$ mice enrolled per arm (individual plots shown in Extended Data Fig.17); data are representative from one of two independent in vivo studies for each PDX line; data are mean \pm s.d. h, Treatment with 10LH and 302LHPC-CARs potently upregulate HLA expression in PDX tumours collected from lone mice in each arm reaching tumour burden compared with mice treated with untransduced T cells (COG-564x collected 11 days after treatment; NBSD collected 14 days after treatment for untransduced cells and 17 days after treatment for $10 \mathrm{LH}$ and $302 \mathrm{LH}$; both tumours collected from one experiment). Created with BioRender.com.

We next treated immunodeficient mice engrafted with HLA-A*24:02 (SKNAS and COG-564x) and HLA-A*23:01 (NBSD) xenografts with $10^{6}$ 10LH- and 302 LH-transduced CAR T cells once tumours reached 100$250 \mathrm{~mm}^{3}$. Both 10LH PC-CAR-treated and 302 LHPC-CAR-treated mice showed complete tumour responses in both HLA-A*24:02 xenografts (Fig. 4g), but only 10LH-treated mice exhibited the response in the HLA-A*23:01 NBSD xenografts. This correlated directly with the relative affinity of these two constructs against the PHOX2B peptide presented on HLA-A*23:01 (Extended Data Fig. 14c), suggesting that a threshold affinity or distinct mode of binding by different scFvs may contribute to the ability to recognize the peptide in slightly altered conformations when presented by different HLA allotypes. We also observed that CAR treatment induced substantial upregulation of MHC in tumours. The COG-564x PDX model was generated from a post mortem blood draw from a patient with high-risk $M Y C N$-amplified neuroblastoma who had suffered multiple relapses, and shows an extremely rapid tumour growth rate in mice. In this experiment, one mouse treated with the 10LH construct had a tumour reach endpoint size of $2 \mathrm{~cm}^{3}$ just one week after PC-CAR T cell therapy and was available for analysis, whereas all other tumours in this arm nearly reached endpoint size and then all regressed (Fig. 4g, Extended Data Figs. 16, 17). The lone COG-564x and 
NBSD tumours that reached endpoint showed significant PC-CAR T cell infiltrate and marked upregulation of $\mathrm{MHC}$ expression compared with endpoint tumours treated with non-transduced CAR T cells (Fig. 4h, Extended Data Fig. 17b). This upregulation is likely to be due to the potent IFN- $\gamma$ release as measured in vitro, suggesting that these therapies can activate $T$ cells at low antigen density to initiate a feed-forward cascade that increases MHC and antigen presentation.

\section{Discussion}

Here we have presented a process for identifying tumour-specific antigens derived from non-mutated oncoproteins, engineering PC-CARs against these tumour self-peptides and screening for cross-reactivity against MHC and the normal immunopeptidome. These methods have resulted in PC-CARs that can induce potent tumour killing across multiple HLA alleles in neuroblastoma and provide a roadmap for addressing the major challenges of therapeutic targeting of intracellular oncoproteins. These approaches demonstrate the value of pairing genomic, transcriptomic, epigenomic and immunopeptidomics datasets of normal and tumour tissues for the discovery of immunotherapy targets, as well as the utility of the ReD system paired with SCRAP to select ultra-rare scFv clones with desired binding and specificity profiles. Targeting of non-immunogenic self-antigens through pMHC-directed PC-CARs can vastly expand the landscape of actionable immunotherapeutic targets and enable the development of personalized immunotherapies in neuroblastoma and other cancers. Owing to the limitations in ionization and canonical search spaces of our immunopeptidomics, the prioritized peptides here are likely to represent only a fraction of potential pMHC complexes available to immunotherapeutic targeting. Neuroblastomas in general, and especially those harbouring $M Y C N$ amplification $^{37}$, exist in a highly immunosuppressive tumour microenvironment such that future iterations of PC-CARs may require additional engineering to enable T cells to navigate to the pMHC target. However, our demonstration of significant upregulation of MHC (and thus target) in our models may help alleviate this therapeutic obstacle.

We also highlight the utility of pairing ShinyNAP with RosettaMHC in identifying HLA allotypes capable of presenting identical peptides in a similar conformation. We suggest that these tools, in addition to matched peptide searches of immunopeptidomics data across multiple tumour samples, have the potential to appreciably expand the identification of tumour-specific peptides presented on multiple HLAs. The potential ability to target these antigens beyond canonical HLA restriction can substantially expand the population of patients eligible to receive each PC-CAR construct and reach underserved populations, but this will need to be verified with other PC-CARs that are in development. The use of the SCRAP algorithm can rapidly exclude constructs with safety liabilities and prioritize those constructs with optimal safety profiles, in contrast to alanine scanning which determines construct-specific cross-reactivities post hoc ${ }^{34}$.

We demonstrate several distinct advantages of PC-CARs for targeting of MHC peptides compared with TCRs: (1) PC-CARs can be used to target essential unmutated oncoproteins by inducing immunogenicity using synthetic peptide-centric receptors; (2) in contrast to TCRs, peptide-centric receptors are not constrained by the germline-encoded CDR1 and CDR2 interactions with MHC and therefore can be engineered such that these binding loops are able to form additional contacts with target peptides independently of the HLA, allowing us to generate receptors with superior peptide selectivity to that of TCRs; and(3) owing to the lack of CDR1 and CDR2 loopinteractions with MHC, PC-CARs can target a peptide presented by multiple HLA alleles, opening up the potential to substantially increase the clinical reach of each construct. Furthermore, PC-CARs may have an advantage over CARs in targeting membrane proteins in their ability to initiate a feed-forward loop of MHC upregulation and increased antigen density. These findings build on recent studies that demonstrate the ability to target neoantigens from mutated TP53 and RAS on MHC using scFv-based approaches and further demonstrate the utility of these approaches in targeting intracellular proteins ${ }^{38,39}$. We expect that the methods presented here will facilitate the discovery of tumour-specific targets in other human cancers with high unmet need and envision a library of scFv-based synthetic immunotherapies that provides population-scale coverage of HLA genotypes for both neoantigens and self-antigens.

\section{Online content}

Any methods, additional references, Nature Research reporting summaries, source data, extended data, supplementary information, acknowledgements, peer review information; details of author contributions and competing interests; and statements of data and code availability are available at https://doi.org/10.1038/s41586-021-04061-6.

1. Pearlman, A. H. et al. Targeting public neoantigens for cancer immunotherapy Nat. Cancer 2, 487-497 (2021)

2. Alexandrov, L. B. et al. Signatures of mutational processes in human cancer. Nature $\mathbf{5 0 0}$ 415-421 (2013).

3. Leko, V. \& Rosenberg, S. A. Identifying and targeting human tumor antigens for T cell-based immunotherapy of solid tumors. Cancer Cell 38, 454-472 (2020).

4. Durbin, A. D. et al. Selective gene dependencies in $M Y C N$-amplified neuroblastoma include the core transcriptional regulatory circuitry. Nat. Genet. 50, 1240-1246 (2018).

5. Majzner, R. G. \& Mackall, C. L. Clinical lessons learned from the first leg of the CAR T cell journey. Nat. Med. 25, 1341-1355 (2019).

6. Tsherniak, A. et al. Defining a cancer dependency map. Cell 170, 564-576.e516 (2017)

7. Yossef, R. et al. Enhanced detection of neoantigen-reactive T cells targeting unique and shared oncogenes for personalized cancer immunotherapy. JCI Insight 3, e122467 (2018). . Yarmarkovich, M. et al. Immunogenicity and immune silence in human cancer. Front. Immunol. 11, 69 (2020)

9. Schumacher, T. N. \& Schreiber, R. D. Neoantigens in cancer immunotherapy. Science $\mathbf{3 4 8}$, 69-74 (2015).

10. Shao, W. et al. The SysteMHC Atlas project. Nucleic Acids Res. 46, D1237-D1247 (2018).

11. Maus, M. V. et al. An MHC-restricted antibody-based chimeric antigen receptor requires TCR-like affinity to maintain antigen specificity. Mol. Ther. Oncolytics 3, 1-9 (2016).

12. Linette, G. P. et al. Cardiovascular toxicity and titin cross-reactivity of affinity-enhanced T cells in myeloma and melanoma. Blood 122, 863-871 (2013).

13. Morgan, R. A. et al. Cancer regression and neurological toxicity following anti-MAGE-A3 TCR gene therapy. J. Immunother. 36, 133-151 (2013).

14. Matthay, K. K. et al. Neuroblastoma. Nat. Rev. Dis. Primers 2, 16078 (2016).

15. Pugh, T. J. et al. The genetic landscape of high-risk neuroblastoma. Nat. Genet. 45, 279-284 (2013).

16. Burr, M. L. et al. An evolutionarily conserved function of polycomb silences the MHC class I antigen presentation pathway and enables immune evasion in cancer. Cancer Cell 36, 385-401.e388 (2019).

17. Aguet, F. et al. The GTEx Consortium atlas of genetic regulatory effects across human tissues. Science 369, 1318-1330 (2020).

18. Rokita, J. L. et al. Genomic profiling of childhood tumor patient-derived xenograft models to enable rational clinical trial design. Cell Rep. 29, 1675-1689.e1679 (2019).

19. Kowalewski, D. J. \& Stevanović, S. Biochemical large-scale identification of MHC class I ligands. Methods Mol. Biol. 960, 145-157 (2013).

20. Bassani-Sternberg, M. et al. Direct identification of clinically relevant neoepitopes presented on native human melanoma tissue by mass spectrometry. Nat. Commun. 7 , 13404 (2016)

21. Ma, X. et al. Pan-cancer genome and transcriptome analyses of 1,699 paediatric leukaemias and solid tumours. Nature 555, 371-376 (2018).

22. Freudenmann, L. K., Marcu, A. \& Stevanović, S. Mapping the tumour human leukocyte antigen (HLA) ligandome by mass spectrometry. Immunology 154, 331-345 (2018).

23. Raabe, E. H. et al. Prevalence and functional consequence of PHOX2B mutations in neuroblastoma. Oncogene 27, 469 (2007)

24. Lee, N. H. et al. Clinical significance of tyrosine hydroxylase mRNA transcripts in peripheral blood at diagnosis in patients with neuroblastoma. Cancer Res. Treat. 48 1399-1407 (2016).

25. Marachelian, A. et al. Expression of five neuroblastoma genes in bone marrow or blood of patients with relapsed/refractory neuroblastoma provides a new biomarker for disease and prognosis. Clin. Cancer Res. 23, 5374-5383 (2017).

26. Toor, J. S. et al. A recurrent mutation in anaplastic lymphoma kinase with distinct neoepitope conformations. Front. Immunol. 9, 99 (2018).

27. Cardoso-Moreira, M. et al. Gene expression across mammalian organ development. Nature 571, 505-509 (2019).

28. Pattyn, A., Morin, X., Cremer, H., Goridis, C. \& Brunet, J.-F. The homeobox gene Phox $2 b$ is essential for the development of autonomic neural crest derivatives. Nature $\mathbf{3 9 9}$ 366-370 (1999).

29. Wang, L. et al. ASCL1 is a MYCN- and LMO1-dependent member of the adrenergic neuroblastoma core regulatory circuitry. Nat. Commun. 10, 5622 (2019).

30. Hata, J. L. et al. Diagnostic utility of PHOX2B in primary and treated neuroblastoma and in neuroblastoma metastatic to the bone marrow. Arch. Pathol. Lab. Med. 139, 543-546 (2015). 


\section{Article}

31. Mosse, Y. P. et al. Germline PHOX2B mutation in hereditary neuroblastoma. Am. J. Hum. Genet. 75, 727-730 (2004)

32. Dharia, N. V. et al. A first-generation pediatric cancer dependency map. Nat. Genet. 53 529-538 (2021).

33. Beasley, M. D., Niven, K. P., Winnall, W. R. \& Kiefel, B. R. Bacterial cytoplasmic display platform retained display $(\mathrm{ReD})$ identifies stable human germline antibody frameworks. Biotechnol. J. 10, 783-789 (2015)

34. Kunert, A., Obenaus, M., Lamers, C. H. J., Blankenstein, T. \& Debets, R. T-cell receptors for clinical therapy: in vitro assessment of toxicity risk. Clin. Cancer Res. 23, 6012-6020 (2017).

35. Rudolph, M. G., Stanfield, R. L. \& Wilson, I. A. How TCRs bind MHCs, peptides, and coreceptors. Annu. Rev. Immunol. 24, 419-466 (2006).

36. Nerli, S. \& Sgourakis, N. G. Structure-based modeling of SARS-CoV-2 peptide/HLA-AO2 antigens. Front. Med. Technol. https://doi.org/10.3389/fmedt.2020.553478 (2020).

37. Asgharzadeh, S. et al. Clinical significance of tumor-associated inflammatory cells in metastatic neuroblastoma. J. Clin. Oncol. 30, 3525-3532 (2012).

38. Douglass, J. et al. Bispecific antibodies targeting mutant RAS neoantigens. Sci. Immunol. , eabd5515 (2021).
39. Hsiue, E. H.-C. et al. Targeting a neoantigen derived from a common TP53 mutation. Science, eabc8697 (2021)

Publisher's note Springer Nature remains neutral with regard to jurisdictional claims in published maps and institutional affiliations.

Open Access This article is licensed under a Creative Commons Attribution 4.0 International License, which permits use, sharing, adaptation, distribution and reproduction in any medium or format, as long as you give appropriate credit to the original author(s) and the source, provide a link to the Creative Commons license and indicate if changes were made. The images or other third party material in this article are included in the article's Creative Commons license, unless indicated otherwise in a credit line to the material. If material is not included in the article's Creative Commons license and your intended use is not permitted by statutory regulation or exceeds the permitted use, you will need to obtain permission directly from the copyright holder. To view a copy of this license visit http://creativecommons.org/licenses/by/4.0/.

(c) The Author(s) 2021 


\section{Methods}

\section{Neuroblastoma samples and cell lines}

Five neuroblastoma cell line xenografts and three patients derived xenografts showing a range of HLA expression by RNA sequencing and immunohistochemistry were selected for the initial immunopeptidomics experiment (Extended Data Table 1). All had whole exome sequencing and single nucleotide polymorphism array data available in addition to RNA-seq data ${ }^{18}$. Eight high-risk tumours with a mean mass of $0.56 \mathrm{~g}$ ranging from $0.17-1.7 \mathrm{~g}$ were obtained from Children's Oncology Group (COG; https://childrensoncologygroup.org/) with matched sequencing from TARGET (https://ocg.cancer.gov/programs/target). Informed consent from each research subject or legal guardian was obtained for each deidentified tumour and blood sample used in this study through the COG neuroblastoma biobanking study ANBLOOB1.

Human-derived neuroblastoma cell lines, including SK-N-AS, SK-N-FI and NB-SD were obtained from the Maris Lab cell line bank. Neuroblastoma cell lines were cultured in RPMI supplemented with $10 \%$ fetal bovine serum (FBS), $100 \mathrm{U} \mathrm{ml}^{-1}$ penicillin, $100 \mathrm{\mu g} \mathrm{ml}^{-1}$ streptomycin, and $2 \mathrm{mML}$-glutamine. Other human cancer cell lines, including Jurkat, SW620, HEPG2 and KATO III were obtained from American Type Culture Collection (ATCC). Jurkat cells were cultured in Iscove's modified Dulbecco's medium (IMDM) supplemented with $10 \%$ FBS,

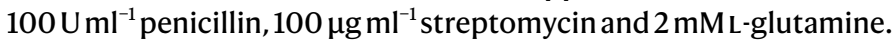
SW620 cells were cultured in RPMI supplemented with $10 \%$ FBS, 100 $\mathrm{U} \mathrm{ml}^{-1}$ penicillin, $100 \mathrm{\mu g} \mathrm{ml}^{-1}$ streptomycin, and $2 \mathrm{mM} \mathrm{L-glutamine.}$ HEPG2 cells were cultured in Eagle's minimum essential medium (EMEM) supplemented with $10 \% \mathrm{FBS}, 100 \mathrm{U} \mathrm{ml}^{-1}$ penicillin, $100 \mathrm{\mu g} \mathrm{ml}^{-1}$ streptomycin and $2 \mathrm{mM}$ L-glutamine. KATO III cells were cultured in IMDM supplemented with $20 \% \mathrm{FBS}, 100 \mathrm{U} \mathrm{ml}^{-1}$ penicillin, $100 \mathrm{\mu g} \mathrm{ml}^{-1}$ streptomycin and $2 \mathrm{mM}$ L-glutamine. Packaging cell lines including Platinum-A cells and HEK 293T cells were obtained from Cell BioLabs and ATCC, respectively. Both packaging cell lines were cultured in DMEM supplemented with $10 \% \mathrm{FBS}, 100 \mathrm{U} \mathrm{ml}^{-1}$ penicillin, $100 \mathrm{\mu g} \mathrm{ml}^{-1}$ streptomycin and $2 \mathrm{mM}$ L-glutamine. All cell lines were grown under humified conditions in $5 \% \mathrm{CO}_{2}$ at $37^{\circ} \mathrm{C}$, and samples were regularly tested for mycoplasma contamination.

\section{Primary human T cells}

Primary human T cells were obtained from anonymous donors through the Human Immunology Core at the University of Pennsylvania (Philadelphia, PA) under a protocol approved by the Children's Hospital of Philadelphia Institutional Review Board. Cells were cultured using AIM-V (Thermo Fisher Scientific) supplemented with 10\% FBS, 100 $\mathrm{U} \mathrm{ml}^{-1}$ penicillin, $100 \mu \mathrm{g} \mathrm{ml}^{-1}$ streptomycin and $2 \mathrm{mML}^{- \text {glutamine under }}$ humified conditions in $5 \% \mathrm{CO}_{2}$ at $37^{\circ} \mathrm{C}$. T cell donors provided informed consent through the University of Pennsylvania Immunology Core

\section{Isolation of HLA ligands by immunoaffinity purification}

HLA class I molecules were isolated using standard immunoaffinity purification as described previously, ${ }^{53,54}$. In brief, cell pellets were lysed in $10 \mathrm{mM}$ CHAPS/PBS (AppliChem/Lonza) containing $1 \times$ protease inhibitor (Complete; Roche). Mouse MHC molecules were removed using a $1 \mathrm{~h}$ immunoaffinity purification with $\mathrm{H}-2 \mathrm{~K}$-specific monoclonal antibody 20-8-4S, covalently linked to CNBr-activated sepharose (GE Healthcare). Remaining HLA molecules were purified overnight using the pan-HLA class I-specific monoclonal antibody W6/32 or a mix of the pan-HLA class II-specific monoclonal antibody Tü39 and the HLA-DR-specific monoclonal antibody L243, covalently linked to $\mathrm{CNBr}$-activated Sepharose. MHC-peptide complexes were eluted by repeated addition of $0.2 \%$ trifluoroacetic acid (Merck). Elution fractions $\mathrm{E} 1-\mathrm{E} 4$ were pooled, and free $\mathrm{MHC}$ ligands were isolated by ultrafiltration using centrifugal filter units (Amicon; Merck Millipore). MHC ligands were extracted and desalted from the filtrate using ZipTip C18 pipette tips (Merck Millipore). Extracted peptides were eluted in
$35 \mu$ l of acetonitrile (Merck)/0.1\% trifluoroacetic acid, centrifuged to complete dryness and resuspended in $25 \mu \mathrm{l}$ of $1 \%$ acetonitrile/0.05\% trifluoroacetic acid. Samples were stored at $-20^{\circ} \mathrm{C}$ until analysis by LC-MS/MS.

\section{Analysis of HLA ligands by LC-MS/MS}

Peptide samples were separated by reversed-phase liquid chromatography (nanoUHPLC, UltiMate 3000 RSLCnano, Dionex) and subsequently analysed in an on-line coupled Orbitrap Fusion Lumos (Thermo Fisher Scientific). Samples were analysed in three technical replicates. Sample volumes of $5 \mu \mathrm{l}$ (sample shares of $20 \%$ ) were injected onto a $75 \mu \mathrm{m} \times 2 \mathrm{~cm}$ trapping column (Acclaim PepMap RSLC, Dionex) at $4 \mu \mathrm{min}^{-1}$ for 5.75 min. Peptide separation was subsequently performed at $50^{\circ} \mathrm{C}$ and a flow rate of $300 \mathrm{nl} \mathrm{min}^{-1}$ on a $50 \mu \mathrm{m} \times 25 \mathrm{~cm}$ separation column (Acclaim PepMap RSLC, Dionex) applying a gradient ranging from $2.4-32.0 \%$ of acetonitrile over the course of 90 min. Eluting peptides were ionized by nanospray ionization and analysed in the mass spectrometer implementing the TopSpeed method. Survey scans were generated in the Orbitrap at a resolution of 120,000. Precursor ions were isolated in the quadrupole, fragmented by collision induced dissociation (CID) in the dual-pressure linear ion trap for MHC class I-purified peptides. Finally, fragment ions were recorded in the Orbitrap. An AGC target of $1.5 \times 10^{5}$ and a max injection time of 50ms was used for MS1. An AGC target of $7 \times 10^{4}$ and a max injection time of $150 \mathrm{~ms}$ was used for MS2. The collision energy for CID fragmentation was $35 \%$. For fragmentation mass ranges were limited to $400-650 \mathrm{~m} / \mathrm{z}$ with charge states $2+$ and $3+$ for MHC class I.

Synthetic peptides were analyzed using a thirty minute gradient due to the simplicity of the sample. Full scan was acquired in the Orbitrap with a scan range of $300-1200$ at 120,000 resolution. The automatic gain control (AGC) target was $5.0 \times 10^{5}$ with a maximum injection time of $50 \mathrm{~ms}$. Precursor ions were isolated in the quadropole, fragmented (CID, HCD and ETD) and analysed in the Orbitrap. MS2 were also acquired in the Orbitrap with 30,000 resolution, collision energy of $35 \%$, AGC of $5 \times 10^{4}$, and maximum injection time of $150 \mathrm{~ms}$. Since the discovery analysis were completed using CID, synthetic peptides fragmented with CID were compared for validation.

The mass spectrometry proteomics data have been deposited to the ProteomeXchange Consortium via the $\mathrm{PRIDE}^{40}$ partner repository with the dataset identifier PXD027182 and 10.6019/PXD027182

\section{HLA typing}

FASTQ files from TARGET DNA and RNA-seq and RNA-seq data from cell lines were processed using PHLAT algorithm, as we previously validated on 15 HLA alleles ${ }^{8}$.

\section{Database search and spectral annotation}

Data were processed against the human proteome as compiled in the Swiss-Prot database (https://uniprot.org; 27 May 2021;20,395 reviewed protein sequences contained) using the SequestHT algorithm ${ }^{3}$ in Proteome Discoverer (v2.1, Thermo Fisher Scientific) software. Precursor mass tolerance was set to $5 \mathrm{ppm}$, fragment mass tolerance to 0.02 Da. Search was not restricted to an enzymatic specificity. Oxidized methionine was allowed as a dynamic modification. FDR was determined by the Percolator algorithm based on processing against a decoy database consisting of shuffled sequences. FDR was set to $1 \%$. Peptide lengths were limited to 8-14 amino acids for MHC class I. HLA annotation was performed using NetMHC-4.0 for HLA class I. For peptide matching, data was reprocessed using Proteome Discoverer (v2.4, Thermo Fisher Scientific) using the same parameters but with the addition of the feature mapper node to allow peptide matching between samples. Synthetic peptides were searched using a similar approach but Percolator was replaced with the fixed value PSM validator due to the simplicity of the synthetic peptide sample. Gene Ontology analyses were performed using PANTHER ${ }^{41}$ (http://geneontology.org/) and $P$-values were calculated using Fischer's exact test. 


\section{HLA binding predictions}

HLA binding predictions were performed using NetMHC-4. $0^{42}$, NetMHCpan $4.1^{43}$, and HLAthena ${ }^{44}$ on HLA class I.

\section{scFv biopanning and CAR design}

scFv binders against MHC presented peptides were retrieved from a large $\left(2 \times 10^{10}\right)$ naive phage display scFv library ${ }^{45}$. A competitive panning process was developed to identify specific binders targeting the pMHC based on previous protocols ${ }^{46}$. Biotinylated pMHC monomers (target antigens) and non-biotinyated tetramers (decoy competitors) were obtained from the NIH tetramer core facility. $10^{12}$ copies of phages were depleted against magnetic beads (Invitrogen Dynabeads MyOne Streptavidin T1) for $30 \mathrm{~min}$ before incubation for $1.5 \mathrm{~h}$ with $5 \mu \mathrm{g}$ biotinylated pep-MHC conjugated beads in the presence of $20 \mu \mathrm{g}$ irrelevant decoy competitors. After incubation, the beads were washed with PBS containing $0.05 \%$ Tween-20 (PBST buffer) 5 times followed by two PBS washes. The remaining bound phage were recovered by log-phase TG1 and rescued by M13KO7 helper phage. The amplified phage was collected the next day by $\mathrm{PEG}-\mathrm{NaCl}$ precipitation and used for the next round of panning. The target antigen input was decreased from $5 \mu \mathrm{g}$ for the first round panning to $2 \mu \mathrm{g}$ and $0.5 \mu \mathrm{g}$ for the second and third rounds, respectively, and the washing conditions were more stringent along with the panning rounds. After three rounds of panning, polyclonal phage ELISA was performed to evaluate the enrichment. The TG1 cells from the second and third rounds were randomly picked into 96-well plates for soluble expression-based monoclonal ELISA (semELISA), as described previously ${ }^{46,47}$. Clones producing signals when binding to target antigens and not producing signals when binding the decoy competitors were amplified and sequenced. For protein preparation, these clones were transformed into HB2151 cells for expression, and proteins were purified by one-step Ni-NTA resin affinity. Protein purity and homogeneity were analysed by SDS-PAGE. Protein concentration was measured spectrophotometrically (NanoVue, GE Healthcare). Second-generation CAR constructs were synthesized using scFv sequences with 4-1BB and CD3 3 co-stimulatory domains and cloned into pMP71 vector for screening.

\section{ReD library panning}

The Ruby scFv library ( $>10^{11}$ diversity) was constructed using fully-germline IGLV3-1 and IGLV6-57 scaffolds paired with the IGHV323 scaffold as described ${ }^{33}$, with fully synthetic amino acid diversity in both $\mathrm{V}_{\mathrm{L}}$ and $\mathrm{V}_{\mathrm{H}} \mathrm{CDR} 3$ loops.

The Ruby library was panned for two rounds using PHOX2B (43-51) MHC complex bound to MyOne Streptavidin C1 Dynabeads (ThermoFisher, Cat: 65002). Panned library output were transferred into the ReD cell-display platform ${ }^{33}$ and cells were permeabilized using $0.5 \%$-octyl $\beta$-D-thioglucopyranoside (Anatrace, catalogue (cat.) no. 0314) and labelled using recombinant $\mathrm{PHOX} 2 \mathrm{~B}$ pMHC complex ligated to fluorophores excitable by $405 \mathrm{~nm}$ and $488 \mathrm{~nm}$ lasers. Cells that were positive for target binding were isolated using the FACSMelody sorter (Becton-Dickinson).

After two rounds of positive selection for binding to PHOX2B MHC complex, two further rounds of fluorescence-activated cell sorting (FACS) were conducted using counter-labelled A*24:02 MHC complexes with unrelated peptides. After four rounds of FACS, individual colonies were picked and grown in 96-well plates before scFv induction, cell permeabilization, and $\mathrm{PHOX} 2 \mathrm{~B}$ MHC labelling and detection by CytoFLEX (Beckman Coulter).

Clones that were identified as binding specifically to the PHOX2B (43-51)-MHC complex were sequenced and unique scFvs were expressed as fusions to the AviTag biotinylation motif in Escherichia coli. Biotinylated scFv protein was released by permeabilization with $0.5 \%$-octyl $\beta$-D-thioglucopyranoside and purified to $\sim 90 \%$ purity on Nickel NTA agarose resin (ABT, cat. no. 6BCL-NTANi).

\section{Binding kinetics}

Affinity measurements were performed using a BLItz system (ForteBio) and analysed using BLItz Pro software. Streptavidin biosensors (ForteBio, cat. no.18-5019) were loaded with AviTag-biotinylated scFv, blocked with biotin, washed in PBS and then associated with pMHC ligand in PBS.

\section{Steady-state binding assay}

An equilibrium binding assay to target pMHCs was also established using MyOne Streptavidin C1 Dynabeads. In brief, $50 \mu \mathrm{g}$ of Streptavidin C1 Dynabeads were incubated with excess biotinylated scFv before being blocked with free biotin and washed in PBS. Fluorophore-labelled pMHC complex was added to a concentration of $3.5 \mathrm{nM}$ and incubated for $1 \mathrm{~h}$ at $4{ }^{\circ} \mathrm{C}$ followed by $10 \mathrm{~min}$ at $25^{\circ} \mathrm{C}$. Binding of the free MHC complex to the beads was quantified by the CytoFLEX at $488 \mathrm{~nm}$ (excitation)/525 nm (emission). Binding was normalized to beads without scFv and with unrelated control MHC complex.

This bead-binding assay was used to quantitate the binding of scFv to MHC complexes with alanine-scan substitutions of the PHOX2B peptides as well as to a plate of 95 unrelated 9 mer peptide $A^{*} 24: 02$ MHC complexes and the degree of cross-reactivity of binding of MHC complexes with peptides identified as having high homology to the PHOX2B peptide by eXpitope 2.0 .

\section{Viral production and transduction of Jurkat and primary $T$ cells}

Retrovirus for transduction of Jurkats and primary CD4/8 T cells was produced using Platinum-A (Plat-A) cells, a retroviral packaging cell line. Cells were plated in 6-well plates at $7 \times 10^{5}$ cells per well and transfected with $2.5 \mu$ gof theappropriate TCRorCARconstruct in the retroviralvector pMP71 using Lipofectamine 3000 (Life Technologies, Invitrogen). After $24 \mathrm{~h}$, medium was replaced with IMDM-10\% FBS or AIM-V-10\% FBS for Jurkat cells or primary cells, respectively. Supernatants were collected and filtered with $0.2-\mu \mathrm{m}$ filters after $24 \mathrm{~h}$ incubation.

A second-generation lentiviral system was used to produce replication-deficient lentivirus. The day preceding transfection, 15 million HEK 293T cells were plated in a 15-cm dish. On the day of transfection, $80 \mu \mathrm{l}$ Lipofectamine 3000 (Life Technologies, Invitrogen) was added to $3.5 \mathrm{ml}$ room-temperature Opti-MEM medium (Gibco). Concurrently, $80 \mu \mathrm{l} \mathrm{P} 3000$ reagent (Thermo Fisher Scientific), $12 \mu \mathrm{g}$ psPAX2 (Gag/Pol), 6.5 Hg pMD2.G (VSV-G envelope), and a matching molar quantity of transfer plasmid were added to $3.5 \mathrm{ml}$ room-temperature Opti-MEM medium. Virus supernatant was collected after 24 and $48 \mathrm{~h}$ later and briefly centrifuged at $300 \mathrm{~g}$ and passed through a $0.45-\mu \mathrm{m}$ filter attached to a syringe.

Jurkat cells were plated in 6-well plates pre-treated with $1 \mathrm{ml}$ per well Retronectin $\left(20 \mathrm{mgml}^{-1}\right.$, TakaraBio) at $1 \times 10^{6}$ cellsperwelland spinoculated with $2 \mathrm{ml}$ retroviral supernatant at $800 \mathrm{~g}$ for 30 min at room temperature. After $24 \mathrm{~h}$, cells were collected, and grown in IMDM-10\% FBS.

Primary T cells were thawed and activated in culture for 3 days in the presence of $100 \mathrm{U} \mathrm{ml}^{-1} \mathrm{IL}-2$ and anti-CD3/CD28 beads (Dynabeads, Human T-Activator CD3/CD28, Life Technologies) at a 3:1 bead:T cell ratio. On days 4 and 5, activated cells were plated in 6-well plates pre-treated with $1 \mathrm{ml}$ per well Retronectin ( $20 \mathrm{mg} \mathrm{ml}^{-1}$, Takara Bio) at $1 \times 10^{6}$ cells per well and spinoculated with $2 \mathrm{ml}$ retroviral supernatant at 2,400 rpm for $2 \mathrm{~h}$ at $32^{\circ} \mathrm{C}$. On day 6 , cells were collected and washed, beads were magnetically removed, and cells were expanded in AIM-V$10 \%$ FBS supplemented with $25 \mathrm{U} \mathrm{ml}^{-1}$ IL-2.

Primary human T cells were thawed and activated in culture for 1 day in the presence of $5 \mathrm{ng} \mathrm{ml}^{-1}$ recombinant IL-7, $5 \mathrm{ng} \mathrm{ml}^{-1}$ recombinant IL-15, and anti-CD3/CD28 beads (Dynabeads, Human T-Activator CD3/CD28, Life Technologies) at a 3:1 bead:T cell ratio in G-Rex system vessels (Wilson Wolf). On day 2 , thawed lentiviral vector was added to cultured T cells with $10 \mu \mathrm{g} \mathrm{ml}^{-1}$ Polybrene (Millipore Sigma), and $24 \mathrm{~h}$ later vessels were filled with complete AIM-V medium supplemented 
with indicated concentrations of IL-7 and IL-15. On day 10, cells were collected and washed. Activation beads were magnetically removed, and cell viability was determined before freezing.

Human neuroblastoma cell lines were plated in 6-cm dishes, and $2 \mathrm{ml}$ of thawed lentiviral vector produced with transfer plasmid pLenti-CMV-eGFP-Puro (Addgene plasmid \#17448) was added with $10 \mu \mathrm{g} \mathrm{ml}^{-1}$ Polybrene (Millipore Sigma). Cells were selected for eGFP expression using flow-assisted cell sorting (BD FACSJazz, BD Biosciences) followed by $1 \mu \mathrm{g} \mathrm{ml}^{-1}$ puromycin selection.

\section{sCRAP prediction}

Tumour antigens were compared against the entire normal human proteome on the matched HLA $(85,915,364$ total normal peptides among 84 HLAs). Each residue in the same position of the tumour and human peptides was assigned a score for perfect match, similar amino acid classification or different polarity, scoring 5, 2 or -2, respectively (Extended Data Fig. 12). Similarity scores were calculated based on amino acid classification and hydrophobicity was determined using residues one and three through eight and excluding MHC anchor residues. Next, the maximum normal tissue RPKM values were identified from 1,643 normal tissues in GTEx. Normal peptides were compared to a database of normal tissue immunopeptidomes ${ }^{48}$. The overall cross-reactivity score for each normal peptide was then calculated using the following equation:

$$
\frac{\sum_{i=3}^{n} P_{i}}{b \times E_{\max }}
$$

where $n$ is the peptide length, $P$ is the score of each amino acid of the normal peptide as compared to the tumour antigen, $b$ is the PMHC binding affinity of the normal peptide, and $E_{\max }$ is the maximum normal tissue expression

The algorithm is available at https://marisshiny.research.chop.edu/ sCRAP/.

\section{Tetramer and dextramer staining and flow cytometric analysis}

Surface expression and binding of TCR- and CAR-transduced Jurkat cells and primary $T$ cells was measured by staining with PE- or APC-conjugated dextramers carrying NB antigen peptide-MHC (Immudex). Cells were collected from culture, washed with $2 \mathrm{ml} \mathrm{PBS}$ at $800 \mathrm{~g}$ for $5 \mathrm{~min}$, incubated with $1 \mu \mathrm{l}$ dextramer for $10 \mathrm{~min}$ in the dark, washed again, and resuspended in $300 \mu \mathrm{lPBS}$ for analysis. Typically $5 \times 10^{5}$ cells were used for staining, and analysed on a BD LSR II (BD Biosciences) or an Attune Acoustic Focusing Cytometer (Applied Biosystems, Life Technologies). Flow cytometry data was collected using CytExpert (Beckman Coulter) and FACSDiva (BD Biosciences). Gating strategy for all tetramer and dextramer staining shown in Extended Data Fig. $9 \mathrm{f}$.

\section{Cross-reactivity pMHC screen}

Potential cross-reactive peptides (GenScript) were suspended at a 200 $\mu \mathrm{M}$ working concentration. For each test, $0.5 \mu \mathrm{l}$ of peptide was added to 5 I HLA-A*24:02 empty loadable tetramer (Tetramer Shop) before incubating on ice for $30 \mathrm{~min}$, or using TAPBR peptide exchange as previously described ${ }^{49}$. Following preparation, pMHC tetramers were used to stain cells (described above). CAR construct cross-reactivity values were determined using Jurkat cells transduced with CAR clones followed by staining with HLA-A*24:02 tetramers loaded with cross-reactive peptides. Mean fluorescent intensity was compared across peptides to determine cross reactivity.

\section{Antigen-specific CD8 T cell enrichment and expansion}

Normal donor monocytes were plated on day 1 in 6 -well plates at $5 \times 10^{6}$ cells per well in RPMI-10 FBS supplemented with $10 \mathrm{ng} \mathrm{ml}^{-1}$ IL-4 (Peprotech) and $800 \mathrm{IU} \mathrm{ml}^{-1} \mathrm{GM}-\mathrm{CSF}$ (Peprotech) and incubated at $37^{\circ} \mathrm{C}$ overnight. On day 2 , fresh medium supplemented with $10 \mathrm{ng} \mathrm{ml}^{-1} \mathrm{IL}-4$ and $1,600 \mathrm{IU} \mathrm{ml}^{-1} \mathrm{GM}$-CSF was added to the monocytes and incubated at $37^{\circ} \mathrm{C}$ for another $48 \mathrm{~h}$. On day 4 , non-adherent cells were removed, and immature dendritic cells washed and pulsed with $5 \mu \mathrm{M}$ peptide in AIM-V-10\% FBS supplemented with $10 \mathrm{ng} \mathrm{ml}^{-1} \mathrm{IL}-4,800 \mathrm{IU} \mathrm{ml}^{-1} \mathrm{GM}-\mathrm{CSF}$, $10 \mathrm{ng} \mathrm{ml}^{-1}$ lipopolysaccharide (Sigma-Aldrich), and $100 \mathrm{IU} \mathrm{ml}^{-1} \mathrm{IFN}-\gamma$ (Peprotech) at $37^{\circ} \mathrm{C}$ overnight. Day 1 was repeated on days 4 and 8 to generate dendritic cells for the second and third stimulations on days 8 and 12 , respectively.

On day 5, normal donor-matched CD8+ T cells were enriched using protein kinase inhibitor dasatinib (Sigma-Aldrich), dextramers, and anti-PE or anti-APC beads (Miltenyi Biotec) as previously described ${ }^{50}$. Enriched T cells were co-incubated with the appropriate pulsed dendritic cells in AIM-V-10\% FBS. The day 5 protocol was repeated on days 8 and 12 using dendritic cells generated on days 4 and 8 for the second and third stimulation, respectively. Expanded T cells were validated for antigen-specificity by staining with the appropriate dextramers and for activation marker 41BB/CD137 (BioLegend).

\section{Antigen-specific $T$ cell sorting, sequencing and cloning}

Expanded T cells were stained with CD3, CD8, CD14, CD19, live/dead and matched and mismatched dextramer an single-antigen-specific T cells were sorted using a FACSAria Fusion (BD Biosciences).

Sorted cells were loaded onto $10 \times$ Genomics $5^{\prime} \mathrm{V}(\mathrm{D}) \mathrm{J}$ chips and libraries prepared according to manufacturer protocols. TCR $\alpha / \beta$ amplicons were run on MiSeq using 5,000 reads per cell. Sequencing data were processed using Cell Ranger and analysed using Loupe VDJ Browser. TCR alpha and beta chains were codon optimized and synthesized into bicistronic expression cassettes using engineered cysteine residues in the TCR constant domains, using F2A ribosomal skip sites and furin cleavage sites ${ }^{51}$. TCR cassettes were cloned into pMP71 retroviral vector.

\section{Incucyte cytotoxicity assay}

A total of $0.5 \times 10^{5}$ tumour cell targets were co-incubated with varying ratios of transduced primary cells $\left(5 \times 10^{5}, 2.5 \times 10^{5}, 1 \times 10^{5}, 0.5 \times 10^{5}\right.$ and $2.5 \times 10^{4}$ for $10: 1,5: 1,2: 1,1: 1$ and $1: 2$ effector:target $(\mathrm{E}: \mathrm{T})$ ratios, respectively) in 96 -well plates at $37^{\circ} \mathrm{C}$ in the presence of $0.05 \mu \mathrm{M}$ caspase-3/7 red (Incucyte, Essence BioScience). Plates ran on the Incucyte Zoom or S3 for 24-72 h and measured for apoptosis activity via caspase cleavage and comparison of relative confluency. Following the assay, supernatants were collected for ELISA. Total GFP integrated intensity (Total green calibrated units $\times \mu \mathrm{m}^{2}$ per image) was assessed as a quantitative measure of live, GFP+ tumour cells. Values were normalized to the $t=0$ measurement.

\section{Cytokine secretion assays}

Cell supernatant collected from cell cytotoxicity assays was thawed and plated in triplicate for each condition. IFN- $\gamma$ and IL-2 levels were determined using ELISA kits according to the manufacturer's protocol (BioLegend).

\section{Antigen processing and presentation}

Neuroblastoma cell lines were titrated with H1N5 influenza virus and infectivity was measured by flow cytometry using virus nucleoprotein (NP) antibody. HLA-A2 neuroblastoma cell lines were cultured with either $5 \mu \mathrm{M}$ CEF1 or $50 \mathrm{HAU}$ of H1N5 virus then co-cultured with M1 antigen-specific T cell hybridoma kindly provided by David Canaday ${ }^{52}$. T cell activation was measured using IL-2 ELISA (Abcam).

\section{Expression, refolding, and purification of recombinant peptide and HLA molecules}

HLA-A*02:01 and HLA-A*24:02 constructs for bacterial expression were cloned into $p E T 24 a+$ plasmid. DNA plasmids encoding HLA-A*02:01 (heavy chain), HLA-A*24:02 (heavy chain), and human $\beta 2 \mathrm{M}$ (light chain) were transformed into $E$. coli BL21-DE3 (Novagen), expressed as inclusion bodies and refolded using previously described methods ${ }^{53}$. E. coli cells were grown in autoinduction medium ${ }^{54}$ for (16-18 h). Afterward, 
the $E$. coli cells were collected by centrifugation and resuspended with $25 \mathrm{ml}$ BugBuster (Milipore Sigma) per litre of culture. The cell lysate was sonicated and subsequently pelleted by centrifugation $(5,180 \mathrm{~g}$ for 20 min at $4{ }^{\circ} \mathrm{C}$ ) to collect inclusion bodies. The inclusion bodies were washed with $25 \mathrm{ml}$ of wash buffer (100 mM Tris pH 8.0, 2 mM EDTA, and $0.01 \% \mathrm{v} / \mathrm{v}$ deoxycholate), sonicated, and pelleted by centrifugation. A second wash was done using $25 \mathrm{ml}$ of Tris-EDTA buffer $(100 \mathrm{mM}$ Tris $\mathrm{pH} 8.0$ and $2 \mathrm{mM}$ EDTA). The solution was once again resuspended by sonication then centrifuged. The inclusion bodies were then solubilized by resuspension with $6 \mathrm{ml}$ resuspension buffer (100 mM Tris pH 8.0,2

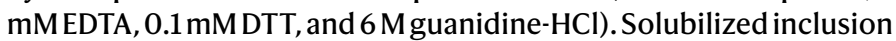
bodies of the heavy and light chain were mixed in a 1:3 molar ratio and then added dropwise over 2 days to 11 of refolding buffer $(100 \mathrm{mM}$ Tris pH 8.0,2 mMEDTA, 0.4 M arginine- $\mathrm{HCl}, 4.9 \mathrm{mM}$ reduced L-glutathione, and $0.57 \mathrm{mM}$ oxidized L-glutathione oxidized) containing $10 \mathrm{mg}$ of synthetic peptide at $>98 \%$ purity confirmed by mass-spectrometry (Genscript). Refolding was allowed to proceed for 4 days at $4{ }^{\circ} \mathrm{C}$ without stirring. Following this incubation period, the refolding mixture was dialyzed into the size-exclusion buffer $(25 \mathrm{mM}$ Tris $\mathrm{pH} 8.0$ and 150 $\mathrm{mM} \mathrm{NaCl}$ ). After dialysis, the sample was concentrated first using a Labscale tangential flow filtration system and then using an Amicon Ultra-15 Centrifugal $10 \mathrm{kDa}$ MWCO Filter Unit (Millipore Sigma), to a final volume of $5 \mathrm{ml}$. Purification was performed using size-exclusion chromatography on a HiLoad $16 / 600$ Superdex 75 column. After size exclusion, the sample was further purified by anion exchange chromatography using a MonoQ $5 / 50 \mathrm{GL}$ column and a $0-100 \%$ gradient of buffer $\mathrm{A}(25 \mathrm{mM}$ Tris $\mathrm{pH} 8.0$ and $50 \mathrm{mM} \mathrm{NaCl})$ and buffer $\mathrm{B}(25 \mathrm{mM}$ Tris $\mathrm{pH} 8.0$ and $1 \mathrm{M} \mathrm{NaCl})$. The purified protein was exhaustively exchanged into $20 \mathrm{mM}$ sodium phosphate $\mathrm{pH} 7.2$ and $50 \mathrm{mM} \mathrm{NaCl}$. The final sample was validated using SDS-PAGE to confirm the formation of a pMHC complex containing both the heavy and light chains.

\section{Differential scanning fluorimetry}

To measure the thermal stability of the pMHc-I molecules, $2.5 \mu \mathrm{M}$ of protein was mixed with $10 \times$ Sypro Orange dye in matched buffer $(20$ $\mathrm{mM}$ sodium phosphate $\mathrm{pH} 7.2,100 \mathrm{mM} \mathrm{NaCl}$ ) in MicroAmp Fast 96-well plates (Applied Biosystems) at a final volume of $50 \mu$ l. Differential scanning fluorimetry was performed using an Applied Biosystems ViiA quantitative PCR machine with excitation and emission wavelengths at $470 \mathrm{~nm}$ and $569 \mathrm{~nm}$, respectively. Thermal stability was measured by increasing the temperature from $25^{\circ} \mathrm{C}$ to $95^{\circ} \mathrm{C}$ at a scan rate of $1^{\circ} \mathrm{C} \mathrm{min}^{-1}$. Melting temperatures $\left(T_{\mathrm{m}}\right)$ were calculated in GraphPad Prism 7 by plotting the first derivative of each melt curve and taking the peak as the $T_{\mathrm{m}}$.

\section{Protein crystallization}

Purified HLA-A*02:01-LLLPLLPPL, HLA-A*02:01-LLPLLPPLSP, HLA-A*02:01-LLPLLPPLSPS, HLA-A*02:01-LLPRLPPL and HLA-A*24:02QYNPIRTTF complexes were used for crystallization. Proteins were concentrated to $10-12 \mathrm{mg} \mathrm{ml}^{-1}$ in $50 \mathrm{mM} \mathrm{NaCl}, 25 \mathrm{mM}$ Tris pH 8.0, and crystal trays were set up using a 1:1 protein-to-buffer ratio at room temperature. Optimal crystals for HLA-A*02:01-LLLPLLPPL, HLA-A*02:01-LLPLLPPLSP and HLA-A*02:01-LLPLLPPLSPS were obtained with $1 \mathrm{M}$ sodium citrate dibasic, $0.1 \mathrm{M}$ sodium cacodylate $\mathrm{pH}$ 6.5. For HLA-A*02:01LLPRLPPL, diffracting crystals were obtained with $0.2 \mathrm{M}$ magnesium chloride, 0.1 M HEPES pH 7.0, 20\% PEG 6000. HLA-A*24:02-QYNPIRTTF diffracting crystals were obtained with $0.1 \mathrm{M} \mathrm{HEPES} \mathrm{pH} 7.0,10 \%$ PEG 6000. Diffraction-quality crystals were collected and incubated from the above conditions plus glycerol as a cryoprotectant and flash-frozen in liquid nitrogen before data collection. All crystals used in this study were grown using the hanging-drop vapour-diffusion method. Data were collected from single crystals under cryogenic condition at Advanced Light Source (beam lines 8.3.1 and 5.0.1). Diffraction images were indexed, integrated, and scaled using MOSFLM and Scala in CCP4 Package $^{55}$. Structures were determined by Phaser $^{56}$ using previously published structures of HLA-A*02:01 (PDB ID 5C07) ${ }^{57}$ and HLA-A*24:02 $(\mathrm{PDB} I D 3 \mathrm{VXN})^{58}$. Model building and refinement were performed using COOT $^{59}$ and Phenix ${ }^{60}$, respectively.

\section{Homology modelling of $\mathrm{p} / \mathrm{HLA}$ complexes using RosettaMHC}

Three-dimensional structural models of HLA-A*23:01, HLA-B*14:02 and HLA-C*07:02 bound to the peptide QYNPIRTTF were generated using RosettaMHC, an in-house method for modeling the $\alpha_{1}$ and $\alpha_{2}$ peptide-binding domains of pMHC-I molecules ${ }^{61}$. In brief, the amino acid sequences of HLA-A*23:01, HLA-B*14:02 and HLA-C*07:02 were first obtained from the IPD-IMGT/HLA Database ${ }^{62}$. The sequence of HLA alleles of interest was aligned against the sequences of 318 HLA curated template structures available in RosettaMHC. For each allele, all candidate templates were selected according to a $70 \%$ sequence identity criterion between aligned residues within the peptide-binding groove (within $3.5 \AA$ A of any peptide heavy atom). Generation of 3D models was performed using a Monte Carlo sampling of sidechain rotamer conformations, followed by gradient-based optimization of all backbone and sidechain degrees of freedom. For each peptide-HLA complex, the top 5 models with the lowest Rosetta binding energy were selected as the final structural ensemble. The quality of the final models was assessed using the Molprobity webserver ${ }^{63}$. Analysis of polar contacts and surface area were performed using the PyMOL Molecular Graphics System, version 2.4.1.

\section{Immunohistochemistry}

CD3 (Dako A0452), PHOX2B (Abcam ab183741), and HLA-ABC (Abcam ab70328) antibodies were used to stain formalin fixed paraffin embedded tissue slides. Staining was performed on a Bond Max automated staining system (Leica Biosystems). The Bond Refine polymer staining kit (Leica Biosystems, DS9800) was used. The standard protocol was followed with the exception of the primary antibody incubation which was extended to $1 \mathrm{~h}$ at room temperature. CD3, PHOX2B and HLA-ABC antibodies were at 1:100, 1:500 and 1:1,200 dilutions, respectively. Antigen retrieval was performed with $\mathrm{E} 1$ (Leica Biosystems) retrieval solution for $20 \mathrm{~min}$ (E2 for PHOX2B). Slides were rinsed, dehydrated through a series of ascending concentrations of ethanol and xylene, then coverslipped. Stained slides were then digitally scanned at $20 \times$ magnification on an Aperio CS-O slide scanner (Leica Biosystems).

\section{Murine PC-CAR T cell preclinical trials}

NOD SCID Gamma (NSG) female (6-8 weeks of age) mice from Jackson Laboratories (stock no. 005557) were used to propagate subcutaneous xenografts. All mice were maintained under barrier conditions and experiments were conducted using protocols and conditions approved by the IACUC at the Children's Hospital of Philadelphia. Treatment was initiated via lateral tail intravenous injection. Dose administered was $100 \mu \mathrm{l}$ per animal of vehicle or CAR T cells as a single treatment. Treatment was administered at weeks $8-10$ when tumour volumes reached $150 \mathrm{~mm}^{3}$ to $250 \mathrm{~mm}^{3}$. Six mice were enrolled per arm based on previous experience and randomized based on tumour size. Mouse technician was blinded to $T$ cell engineering. Tumour volume and survival were monitored bi-weekly measurements until the tumours reached a size of $2.0 \mathrm{~cm}^{3}$ or mice showed signs of graft versus host disease. Animals were removed from study and studies terminated following onset of graft-versus-host disease (GVHD) when animals display hunched posture, rapid breathing, urine staining, weight loss and a body condition score of 2 , as determined by visual inspection. Onset of GVHD defined as urine staining and weight loss of $20 \%$ or weight loss of $10-15 \%$ if accompanied by hunched posture, laboured breathing, or poor body condition.

\section{Statistics and reproducibility}

Box and whisker plot representations of data show the median as centre, and 25th percentile and 75th percentile as bounds of boxes for plots shown in Fig. 1e, Extended Data Figs. 1, $13 \mathrm{~b}$. 


\section{Reporting summary}

Further information on research design is available in the Nature Research Reporting Summary linked to this paper.

\section{Data availability}

Neuroblastoma HLA class I immunopeptidomics data is available through PRIDE under accession PXD027182. Sample files are annotated in Supplementary Table 2. Protein structures are available in the Protein Data Bank under accession codes 7MJ6 (HLA-A*02:01-LLLPLLPPL), 7MJ7 (HLA-A*02:01-LLPLLPPLSP), 7MJ8 (HLA-A*02:01-LLPLLPPLSPS), 7MJ9 (HLA-A*02:01-LLPRLPPL) and 7MJA (HLA-A*24:02-QYNPIRTTF). All other data are availablewithin the articleand supplementary information files, or by request from corresponding author.

\section{Code availability}

The sCRAP algorithm is accessible to the scientific community through a web portal (https://marisshiny.research.chop.edu/sCRAP/).

40. Perez-Riverol, Y. et al. The PRIDE database and related tools and resources in 2019: improving support for quantification data. Nucleic Acids Res. 47, D442-D450 (2019).

41. Mi, H., Muruganujan, A., Ebert, D., Huang, X. \& Thomas, P. D. PANTHER version 14: more genomes, a new PANTHER GO-slim and improvements in enrichment analysis tools. Nucleic Acids Res. 47, D419-D426 (2019).

42. Nielsen, M. et al. Reliable prediction of T-cell epitopes using neural networks with novel sequence representations. Protein Sci. 12, 1007-1017 (2003).

43. Reynisson, B., Alvarez, B., Paul, S., Peters, B. \& Nielsen, M. NetMHCpan-4.1 and NetMHCllpan-4.0: improved predictions of MHC antigen presentation by concurrent motif deconvolution and integration of MS MHC eluted ligand data. Nucleic Acids Res. 48. W449-W454 (2020).

44. Sarkizova, S. et al. A large peptidome dataset improves HLA class I epitope prediction across most of the human population. Nat. Biotechnol. 38, 199-209 (2020).

45. Zhu, Z. \& Dimitrov, D. S. Construction of a large naive human phage-displayed Fab library through one-step cloning. Methods Mol. Biol. 525, 129-142 (2009).

46. Zhang, M.Y. \& Dimitrov, D. S. Sequential antigen panning for selection of broadly crossreactive HIV-1-neutralizing human monoclonal antibodies. Methods Mol. Biol. 562, 143-154 (2009).

47. Chen, W., Xiao, X., Wang, Y., Zhu, Z. \& Dimitrov, D. S. Bifunctional fusion proteins of the human engineered antibody domain $\mathrm{m} 36$ with human soluble $\mathrm{CD} 4$ are potent inhibitors of diverse HIV-1 isolates. Antiviral Res. 88, 107-115 (2010).

48. Shao, W., Caron, E., Pedrioli, P. \& Aebersold, R. in Bioinformatics for Cancer Immunotherapy: Methods and Protocols (ed. Boegel, S.) 173-181 (Springer, 2020).

49. Overall, S. A. et al. High throughput pMHC-I tetramer library production using chaperone-mediated peptide exchange. Nat. Commun. 11, 1909 (2020).

50. Dolton, G. et al. Optimized peptide-MHC multimer protocols for detection and isolation of autoimmune T-cells. Front. Immunol. 9, 1378-1378 (2018).

51. Yang, S. et al. Development of optimal bicistronic lentiviral vectors facilitates high-level TCR gene expression and robust tumor cell recognition. Gene Ther. 15, 1411-1423 (2008).

52. Canaday, D. H. Production of $\mathrm{CD}^{+}$and $\mathrm{CD} 8^{+} \mathrm{T}$ cell hybridomas. Methods Mol. Biol. 960 , 297-307 (2013).

53. Garboczi, D. N., Hung, D. T. \& Wiley, D. C. HLA-A2-peptide complexes: refolding and crystallization of molecules expressed in Escherichia coli and complexed with single antigenic peptides. Proc. Natl Acad. Sci. USA 89, 3429-3433 (1992).

54. Studier, F. W. Stable expression clones and auto-induction for protein production in E. coli. Methods Mol. Biol. 1091, 17-32 (2014).
55. Winn, M. D. et al. Overview of the CCP4 suite and current developments. Acta Crystallogr. D 67, 235-242 (2011).

56. McCoy, A. J. et al. Phaser crystallographic software. J. Appl. Crystallogr. 40, 658-674 (2007).

57. Cole, D. K. et al. Hotspot autoimmune T cell receptor binding underlies pathogen and insulin peptide cross-reactivity. J. Clin. Invest. 126, 2191-2204 (2016).

58. Shimizu, A. et al. Structure of TCR and antigen complexes at an immunodominant CTL epitope in HIV-1 infection. Sci. Rep. 3, 3097 (2013).

59. Emsley, P. \& Cowtan, K. Coot: model-building tools for molecular graphics. Acta Crystallogr. D 60, 2126-2132 (2004).

60. Adams, P. D. et al. PHENIX: a comprehensive Python-based system for macromolecular structure solution. Acta Crystallogr. D 66, 213-221 (2010).

61. Nerli, S. \& Sgourakis, N. G. Structure-based modeling of SARS-CoV-2 peptide/HLA-AO2 antigens. Front. Med. Technol. https://doi.org/10.3389/fmedt.2020.553478 (2020).

62. Maccari, G. et al. IPD-MHC 2.0: an improved inter-species database for the study of the major histocompatibility complex. Nucleic Acids Res. 45, D860-D864 (2017).

63. Davis, I. W. et al. MolProbity: all-atom contacts and structure validation for proteins and nucleic acids. Nucleic Acids Res. 35, W375-W383 (2007).

64. Andreatta, M., Lund, O. \& Nielsen, M. Simultaneous alignment and clustering of peptide data using a Gibbs sampling approach. Bioinformatics 29, 8-14 (2012).

65. Alvarez, B. et al. NNAlign_MA; MHC peptidome deconvolution for accurate MHC binding motif characterization and improved T-cell epitope predictions. Mol. Cell Proteomics 18, 2459-2477 (2019).

66. Sarkizova, S. et al. A large peptidome dataset improves HLA class I epitope prediction across most of the human population. Nat. Biotechnol. 38, 199-209 (2020).

Acknowledgements This work was supported by a St Baldrick's Foundation-Stand Up to Cancer Dream Team Translational Research Grant (SU2C-AACR-DT-27-17). The St. Baldrick's Foundation collaborates with Stand Up To Cancer. Research Grants are administered by the American Association for Cancer Research, the Scientific Partner of SU2C. Stand Up To Cancer is a program of the Entertainment Industry Foundation administered by the American Association for Cancer Research (J.M.M.). This work was also supported by NIH grants U54 CA232568 as part of the Beau Biden Cancer Moonshot Program (J.M.M.) and NIH R35 CA220500 (J.M.M.), R01 Al143997 (N.G.S.), R35 GM125034 (N.G.S), the Science Center Quod Erat Demonstrandum (QED) program at the Philadelphia Science Center (J.M.M. and M.Y.), the Children's Hospital of Philadelphia Cell and Gene Therapy Collaborative (J.M.M. and M.Y.), and the Giulio D'Angio Endowed Chair (J.M.M.). Diffraction data for protein crystallography were collected at the Advanced Light Source, which is supported by the Director, Office of Science, Office of Basic Energy Sciences, and U.S. Department of Energy, under contract DE-ACO2$05 \mathrm{CH} 11231$. We thank D. Canaday, A. Wolpaw, E. Hooijberg and H. Goldstein for providing reagents. BioRender.com was used in generating figures.

Author contributions Conceptualization: M.Y. Methodology: M.Y., Q.F.M., J.W., H.S., T.N., E.R., M.d.M., D.M., K.R.B., H.T., L.E., S.T., S.N., A.W., R.W., M.C., D.M., S.S. and D.G. Immunopeptidomics: M.Y. and M.d.M. scFv screening: R.P., N.L.C., W.L., D.S.D. and B.R.K. Algorithm development: M.Y. and A.F. X-ray crystallography: J.S.T., S.T. and M.Y. Structural modelling, H.T. and M.Y. Analysis: M.Y., Q.F.M., H.T., N.K. and J.M.M. Investigation: M.Y. and J.M.M. Funding acquisition: M.Y., N.G.S. and J.M.M. Writing: M.Y. and J.M.M. Supervision: M.Y. and J.M.M.

Competing interests J.M.M. and M.Y. have equity stake in Tantigen Bio Inc. and HuLA Therapeutics. Both companies have interest in commercializing technologies described herein. B.R.K. is an employee of and has equity interests in Myrio Therapeutics. R.P. and N.L.C. are employees of Myrio Therapeutics. J.M.M., M.Y. and B.R.K. are co-inventors on a patent filed regarding the $\mathrm{PHOX} 2 \mathrm{~B}$ PC-CAR.

\section{Additional information}

Supplementary information The online version contains supplementary material available at https://doi.org/10.1038/s41586-021-04061-6.

Correspondence and requests for materials should be addressed to John M. Maris. Peer review information Nature thanks Lélia Delamarre, Naomi Taylor and the other, anonymous, reviewer(s) for their contribution to the peer review of this work. Peer reviewer reports are available.

Reprints and permissions information is available at http://www.nature.com/reprints. 

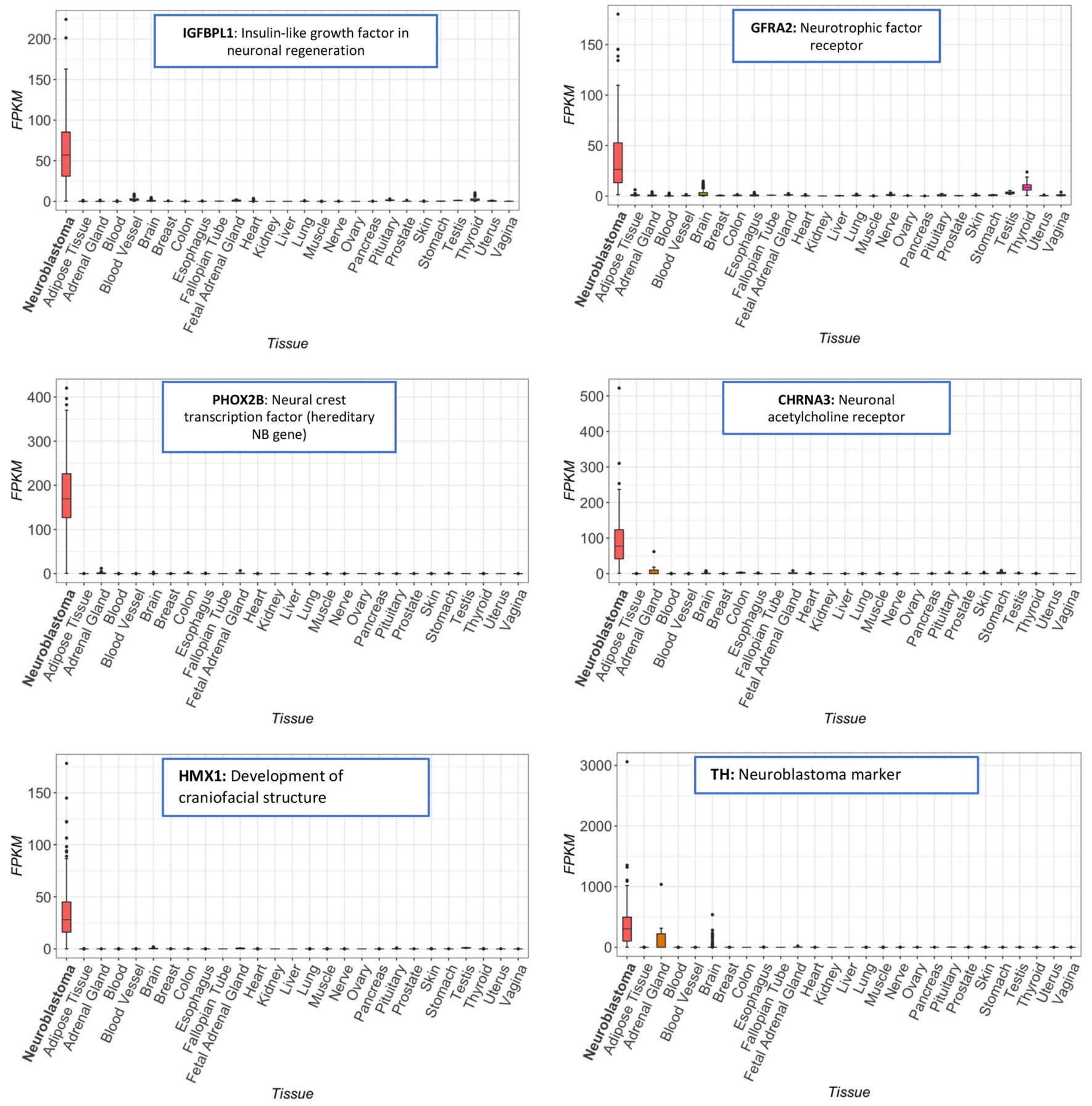

Extended Data Fig. 1 Prioritized peptides from immunopeptidome are highly differentially expressed in neuroblastoma. Differential expression between 153 neuroblastoma tumors from TARGET compared to 1643 normal tissues in GTEx, as described in Fig.1e. 

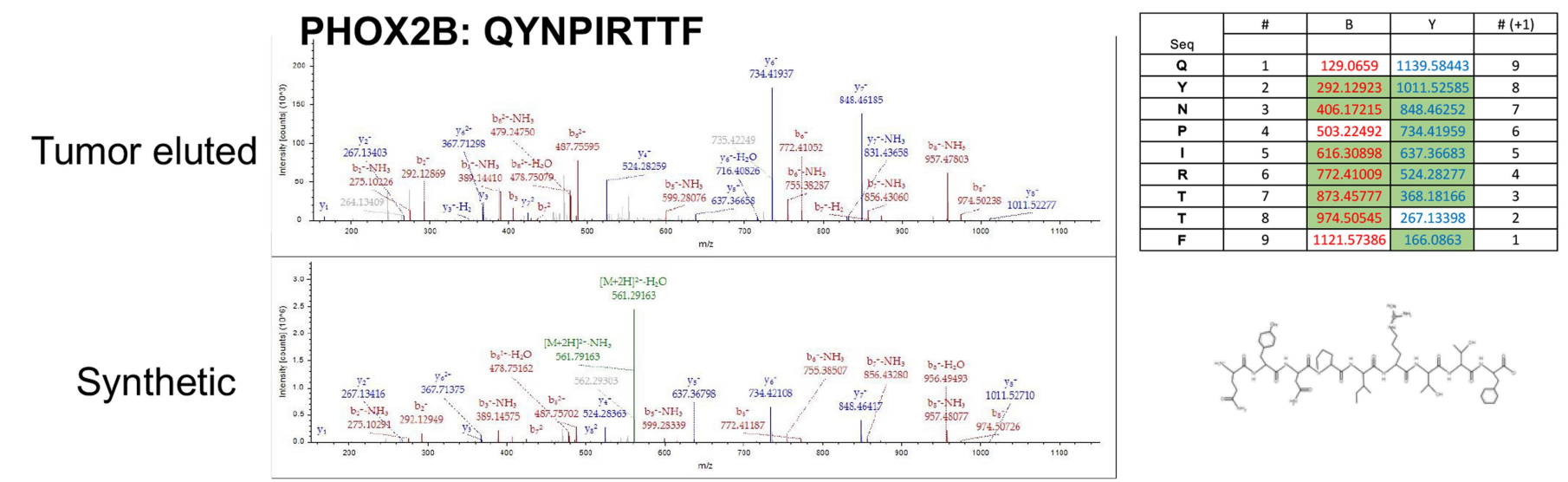

\section{GFRA2: FLDETLRSLA}
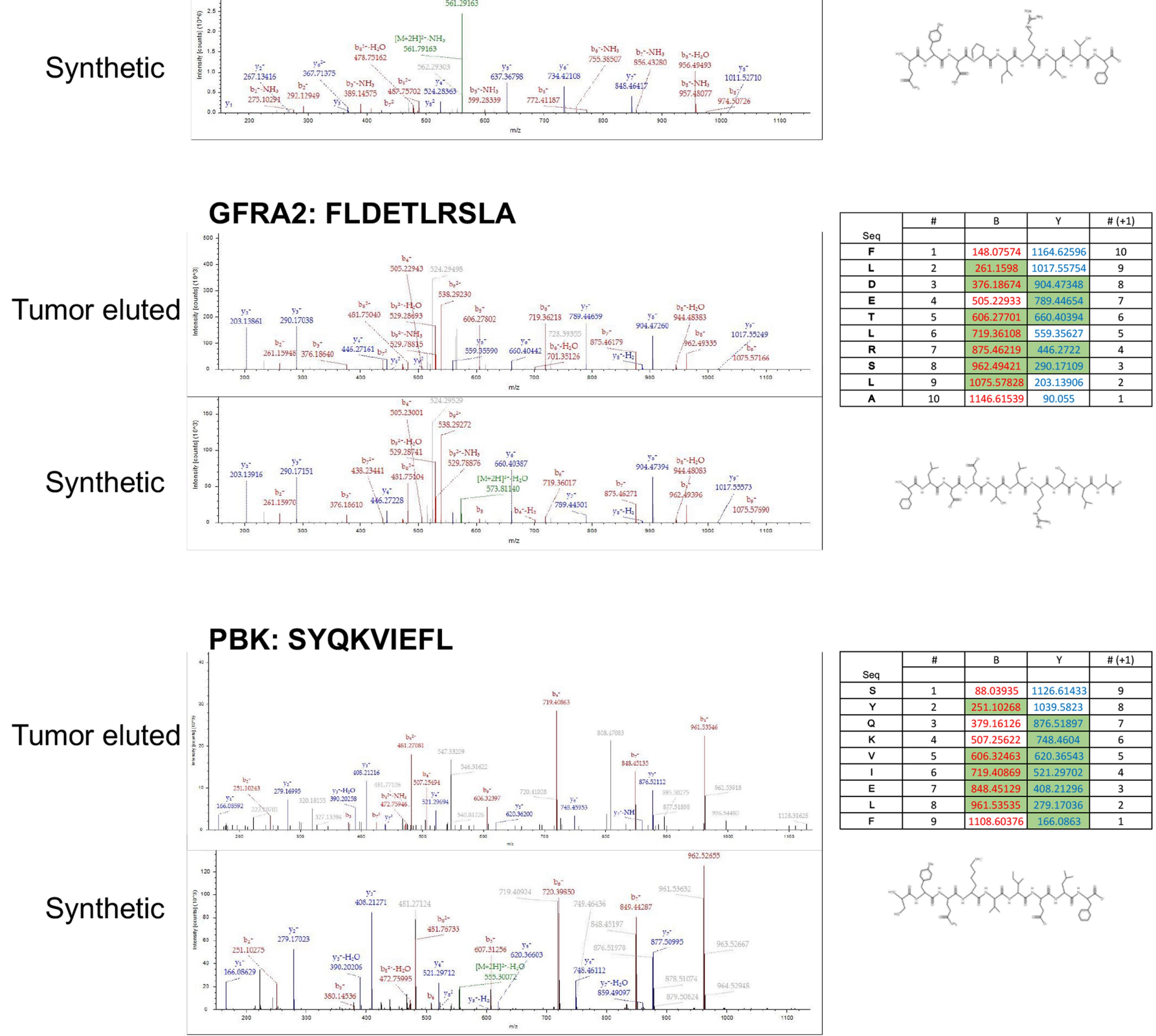

Extended Data Fig. 2 | Validation of antigens discovered by immunopeptidomics using LC/MS/MS of synthetic peptide. Peptide sequences imputed from immunopeptidomics spectra were synthesized and LC/MS/MS was under matched conditions. Synthetic peptides show complete concordance with tumor-eluted peptides across all b and y ions; masses highlighted in green represent detected peaks corresponding to matched $\mathrm{b}$ and $\mathrm{y}$ ions found in both tumor-eluted peptide spectra and synthetic peptide spectra. 


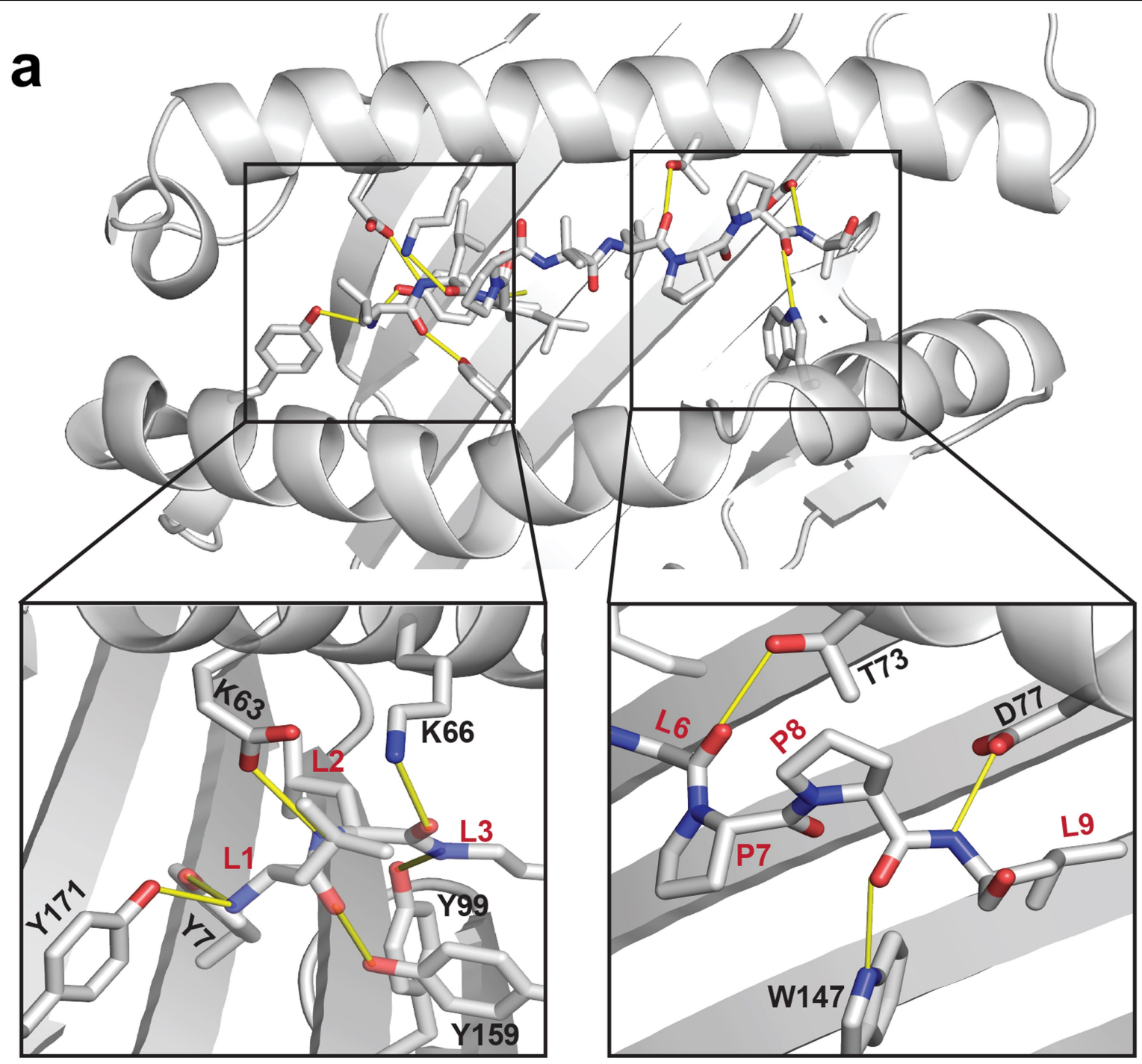

b

Differential Scanning Fluorimetry
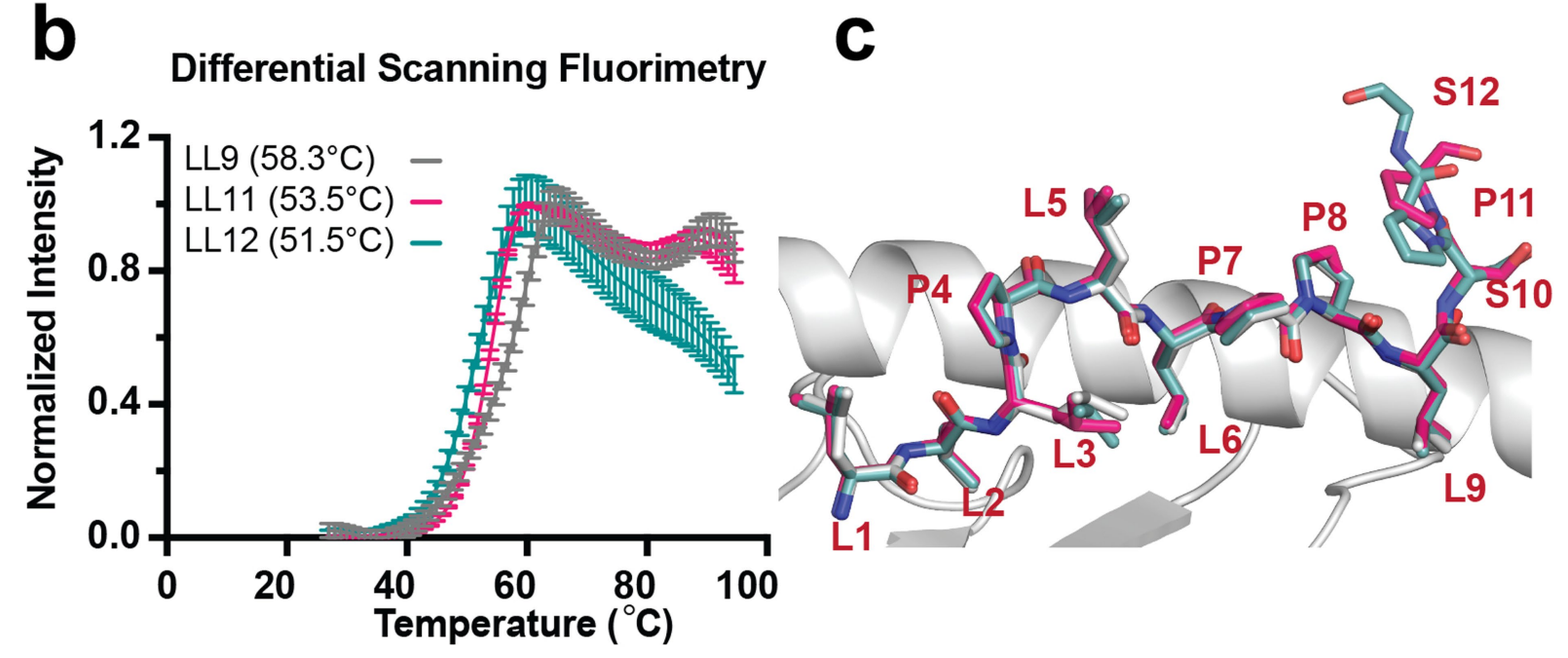

corresponding melting temperature of the resulting peptide/MHC-I

Extended Data Fig. 3 | Crystal structures solved for IGFBPL1 presented as three distinct peptides on HLA-A2. Crystallographic analysis of IGFBPL1 peptide bound to HLA-A*02:01. a. X-ray structure of HLA-A*02:01 presenting the IGFBPL1 nonameric peptide (LLLPLLPPL), where yellow lines represent polar contacts between the HLA groove and peptide. b. Differential Scanning Fluorimetry (DSF) of HLA-A*02:01 refolded with IGFBPL1 peptides of different complexes. Mean of triplicate samples reported with error bars representing SD. c. Overlay of IGFBPL19mer, 11mer, and 12mer in MHC groove reveals that core peptides and anchor residues are maintained across peptides of varying length, and that additional amino acids in the $11 \mathrm{mer}$ and 12 mer protrude at $C$ terminus downstream of the $L 9$ anchor position. 


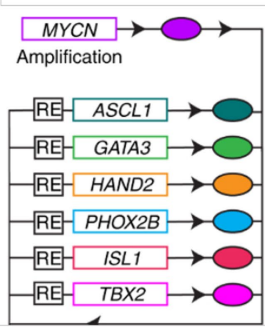

(Wang et al., 2019) b

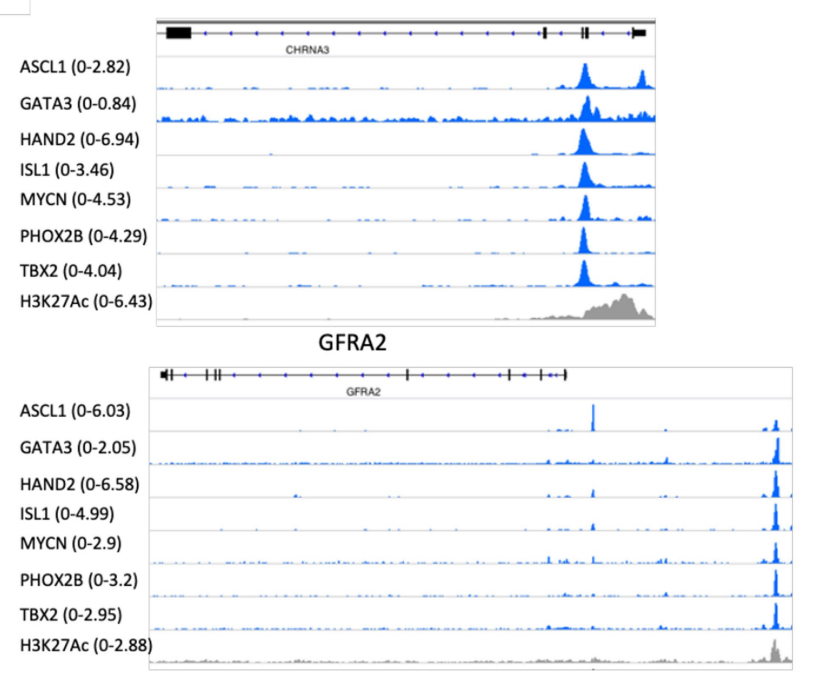

PHOX2B

ASCL1 (0-2.61)

GATA3 (0-1.08)

HAND2 (0-3.19)

ISL1 (0-2.79)

MYCN (0-1.67)

PHOX2B (0-4.44)

$\operatorname{TBX2}(0-1.43)$

H3K27AC (0-3.74)

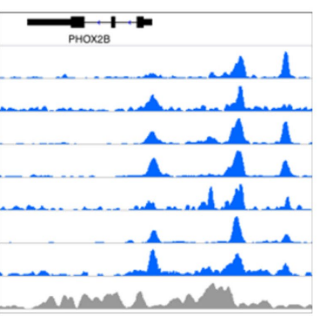

IGFBPL1
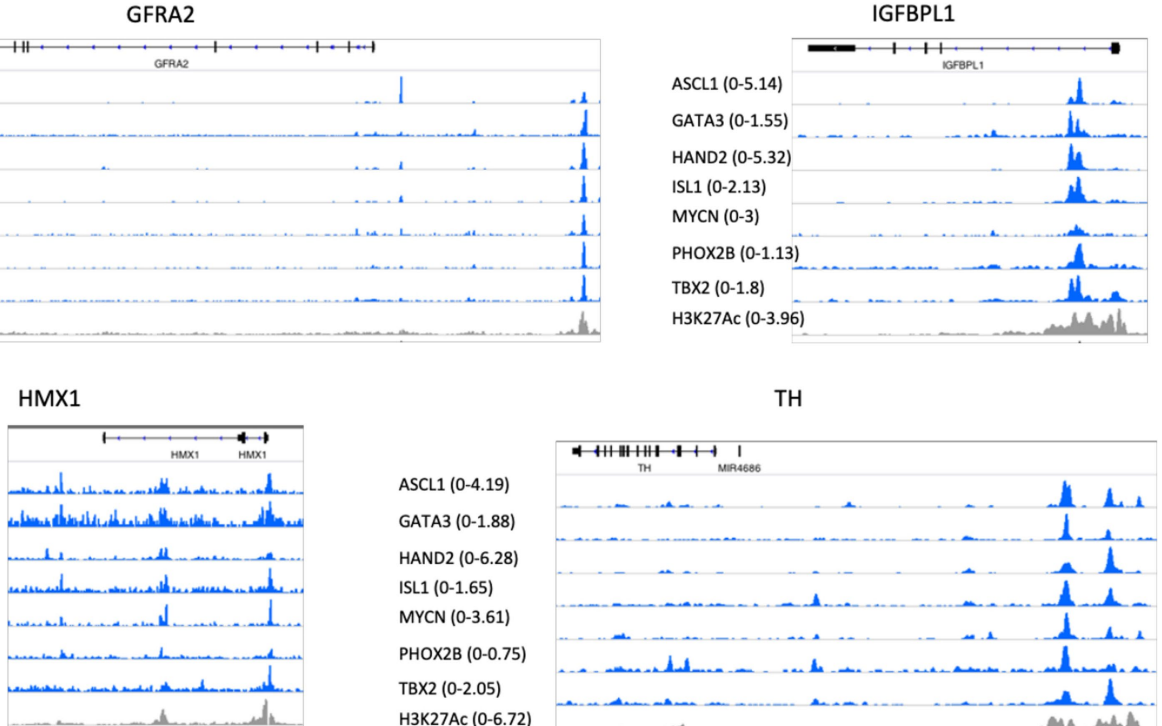

TH

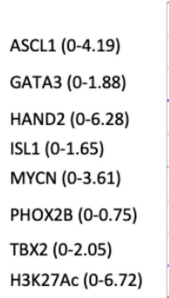

Extended Data Fig. 4 | Tumor antigens derived from parent genes are under control of neuroblastoma core regulatory circuit. a. Illustration of core-regulatory circuit in which transcription factors bind to one another's promoters, acting as feed-forward loop $\mathbf{b}$. ChIP Seq data at prioritized neuroblastoma antigens parent gene loci are bound by each of the 7 coreregulatory circuit (CRC) proteins MYCN, ASCL1, GATA3, HAND2, PHOX2B, ISL1, and TBX2. All CRC binding sites are associated with a H3K27Ac super-enhancer element. 


\section{Article}

a

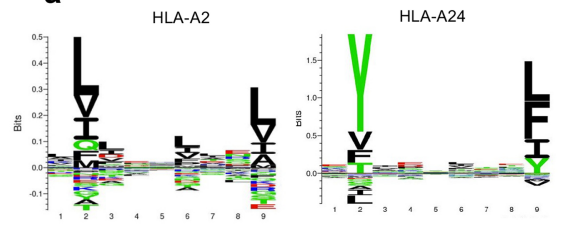

b
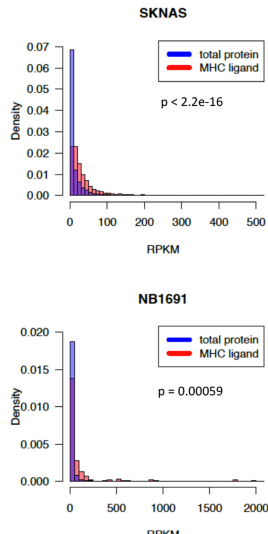

EBC1

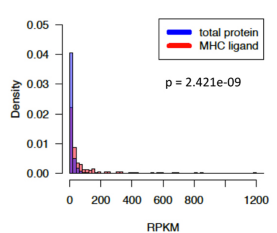

NB1771

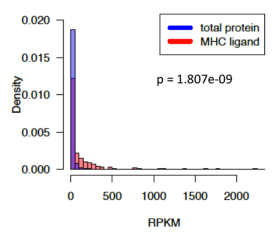

C

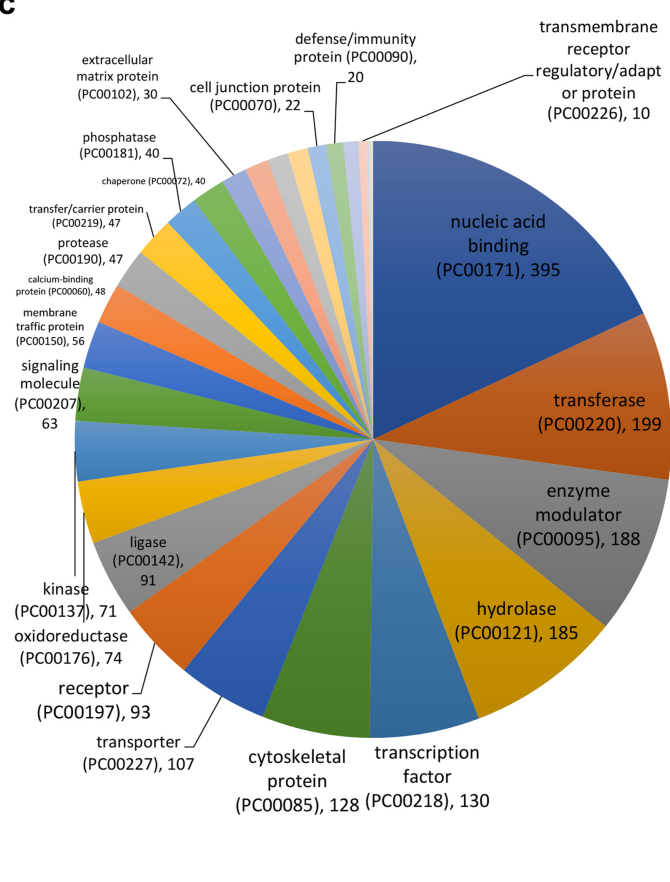

Under-represented

\begin{tabular}{|l|r|}
\hline Gene Ontology & p-val \\
\hline receptor (PC00197) & $2.30 \mathrm{E}-10$ \\
\hline G-protein coupled receptor (PC00021) & $2.73 \mathrm{E}-10$ \\
\hline defense/immunity protein (PC00090) & $1.83 \mathrm{E}-07$ \\
\hline signaling molecule (PCO0207) & $1.17 \mathrm{E}-06$ \\
\hline zinc finger transcription factor (PC00244) & $6.29 \mathrm{E}-05$ \\
\hline
\end{tabular}

peptides of the neuroblastoma immunopeptidome compared to the cellular transcriptome. The most significantly enriched ontology groups are nucleic acid binding proteins (RNA binding protein ontology $\mathrm{p}=1.22 \mathrm{e}^{-25}$ and nucleic acid binding ontology $\mathrm{p}=9.47 \mathrm{e}^{-17} ;$ Fischer's Exact test).

a. Logo plots of peptides eluted from HLA-A2 and HLA-A24 show canonical binding motifs for both alleles ${ }^{64} . \mathbf{b}$. Peptides detected by immunopeptidomics are enriched for highly expressed parent genes as compared to the entire cellular transcriptome. c. Gene ontology analysis of the parent genes of 
a $\mathrm{PHOX} 2 \mathrm{~B}$

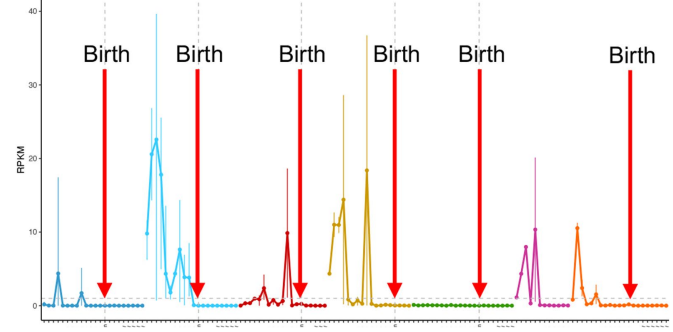

c IGFBPL1

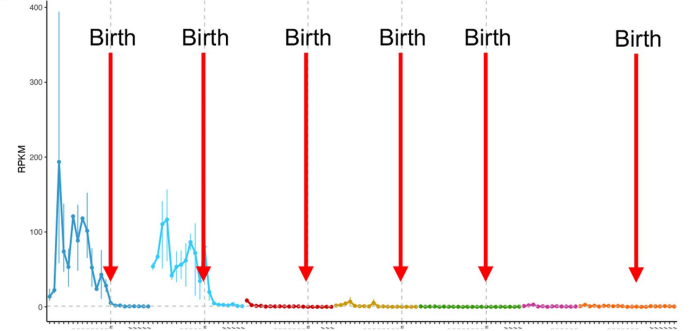

e

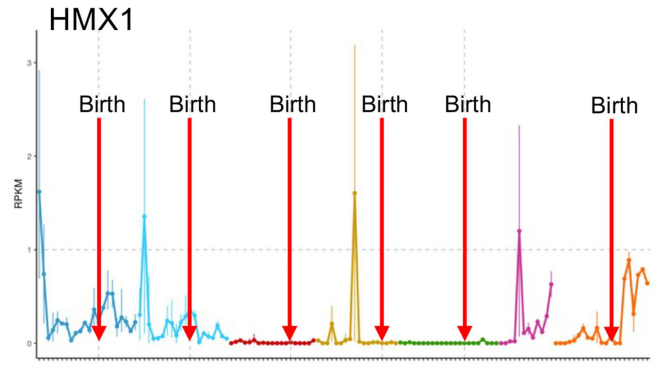

Extended Data Fig. 6 | Prioritized antigen parent genes are expressed during tissue development and downregulated in normal tissue after birth. a-f. Temporal transcriptomic analysis of prioritized neuroblastoma target antigens reveals high expression during development and downregulation prior to birth in the majority of target genes. Expression shown for each tissue from shown from 4 weeks post-conception to 58-63 years

b

d

$\mathbf{f}$
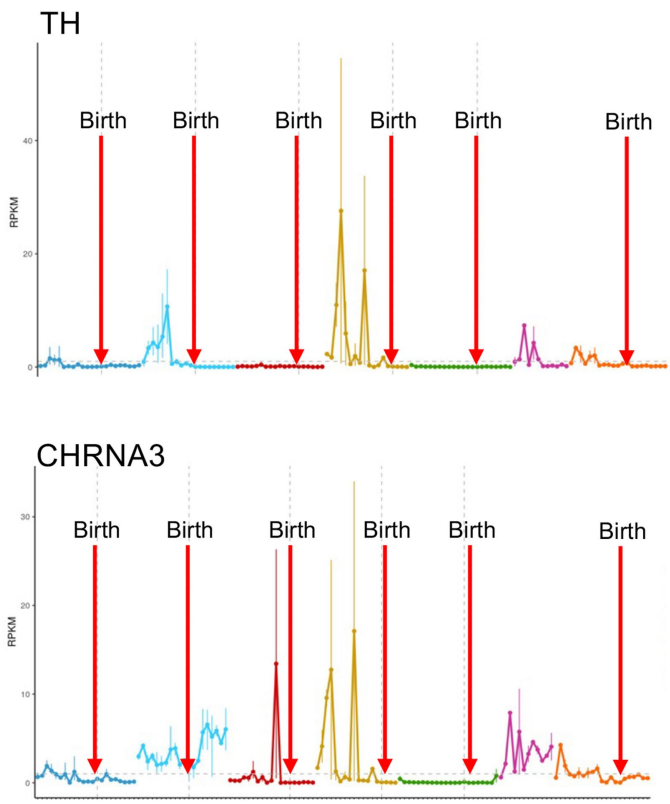

Tissue

$\downarrow$ Brain $\downarrow$ Cerebellum r Heart $\uparrow$ Kidney + Liver + - Ovary + Testis

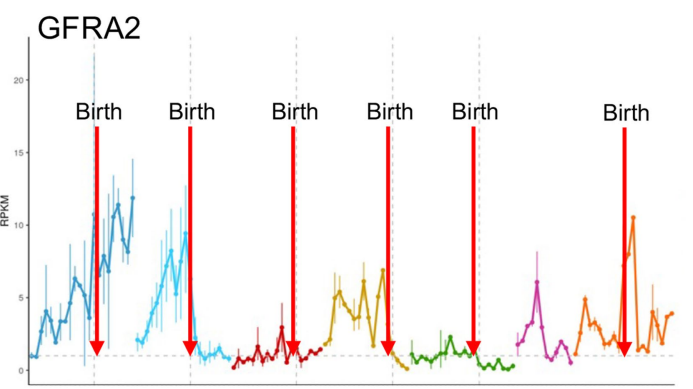

with birth marked by red arrows. PHOX2B (a), TH (b), IGFBPL1 (c), and CHRNA3 (d) exhibit developmentally restricted expression patterns. HMX1 (e) shows expression in testes post-birth. GFRA2 (f) expression in brain may make this target more amenable to targeting by antibody-based therapies that do not cross blood-brain barrier. 
a

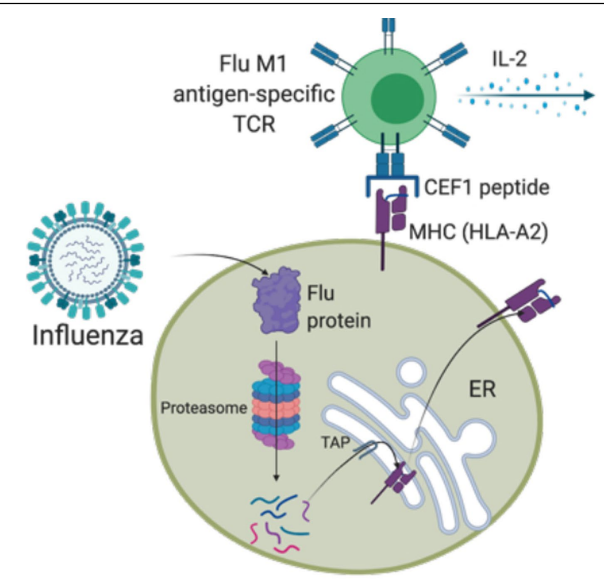

C

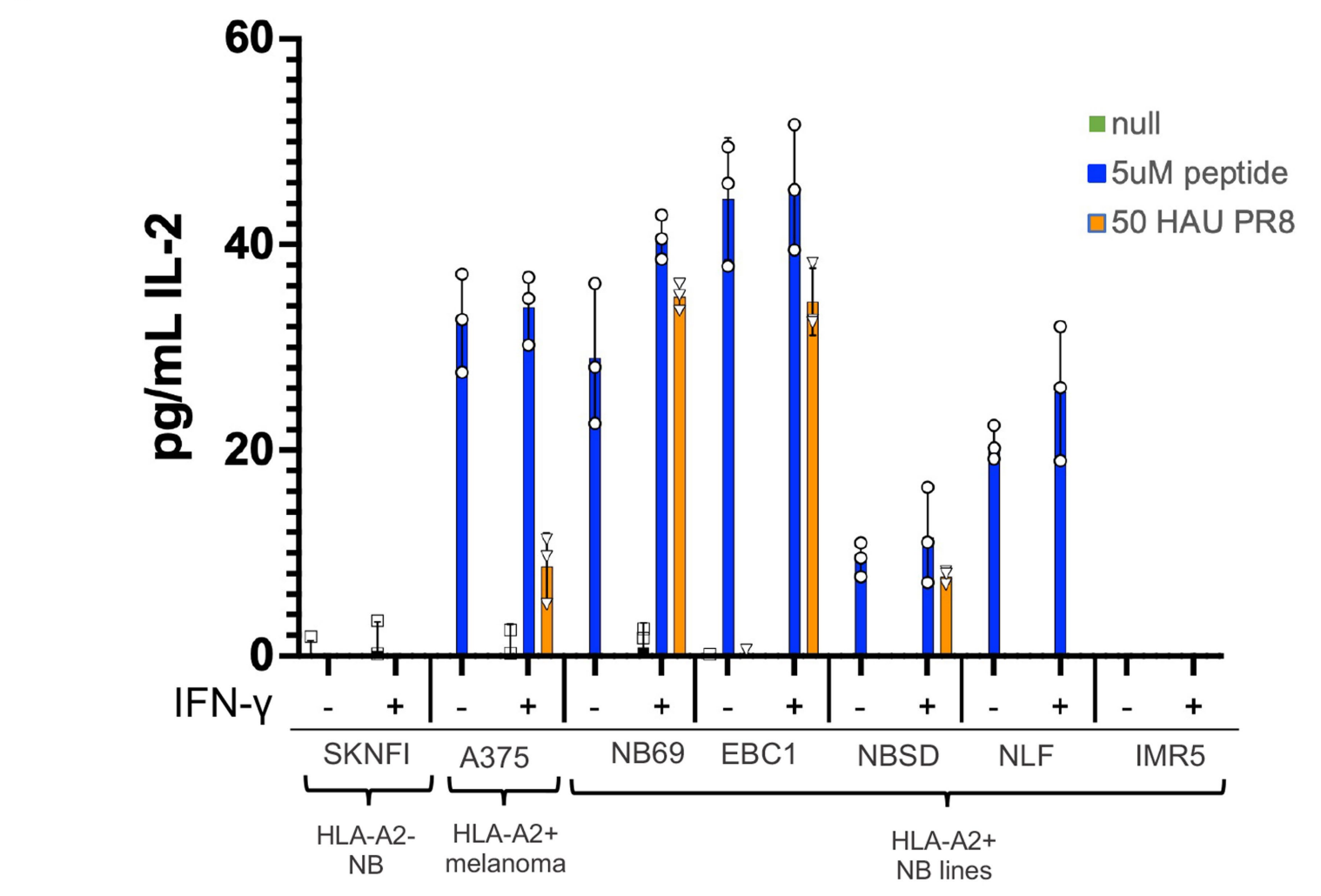

d

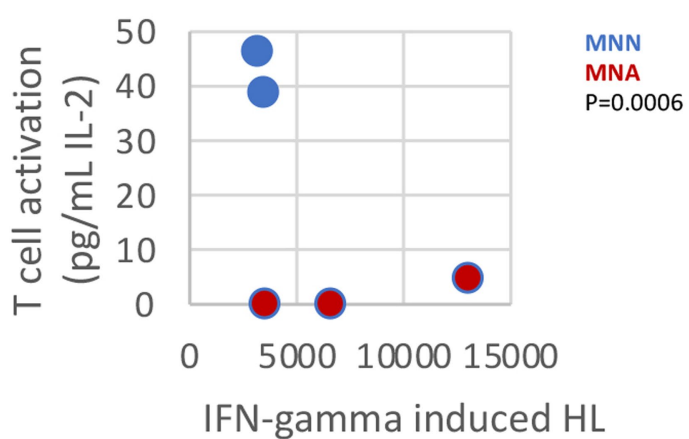

Extended Data Fig. 7 |See next page for caption.

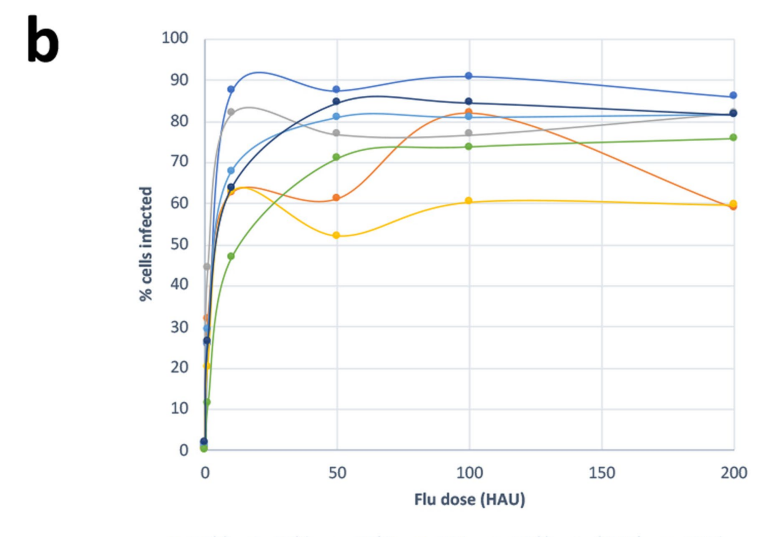

$\rightarrow$ BE2C $\multimap$ EBC1 $\multimap$ NBSD $\multimap$ NB1 $\multimap$ NB69 $\rightarrow$ SKNAS $\rightarrow$ THP1 e

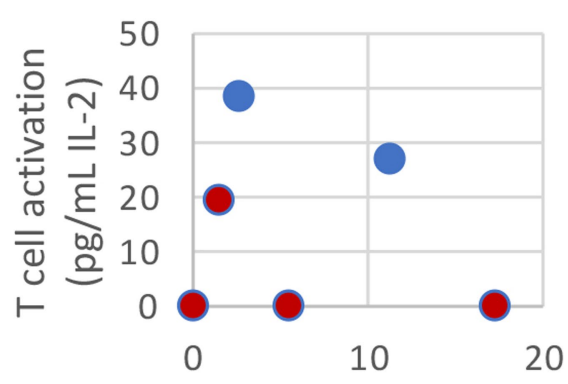

MNN

MNA $P=0.013$ 
Extended Data Fig. 7 | Neuroblastoma antigen processing, presentation, and immunogenicity. a. Schematic of immunogenicity experiment. HLA-A2 neuroblastoma cells were either infected with $\mathrm{H} 1 \mathrm{~N} 5$ or pulsed with synthetic CEF1 peptide and co-cultured with M1 antigen-specific T cell hybridoma line. T cell activation was evaluated by IL-2 release. $\mathbf{b}$. Viral titration of neuroblastoma cells using H1N5 influenza virus measured by FACS staining of viral nucleoprotein expression at 0-200 heamagglutination (HAU). c. Experimental schematic of assay:1) HLA-A2 neuroblastoma cells are pulsed with $5 \mu \mathrm{MCEF} 1$ peptide and co-cultured with M1 antigen-specific hybridoma and IL-2 release is measured by
ELISA; 2) Neuroblastoma cells are infected with 50 HAU of H1N5, co-cultured with $M 1$ antigen-specific hybridoma, measured by IL-2 secretion. Four of seven tested HLA-A*02:01 elicit Thybridoma response when pulsed with CEF1 peptide; three of seven lines induce a response when infected with H1N5 virus. $\mathrm{n}=3$ independent experiments, each performed in triplicate; plots show mean +/-SD. d-e. Thybridoma activation is not associated with HLA expression, but activation is lower in MYCN amplified tumors (MNA) as compared to nonamplified tumors (MNN). Created with BioRender.com. 
a

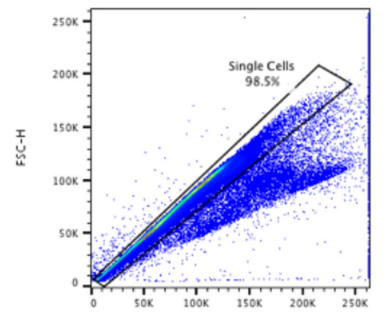

Single cells

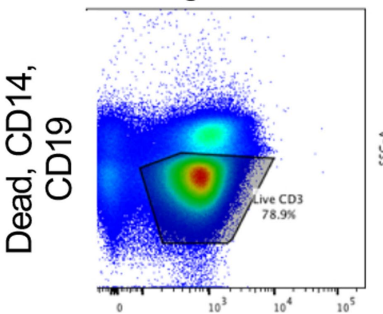

ND046

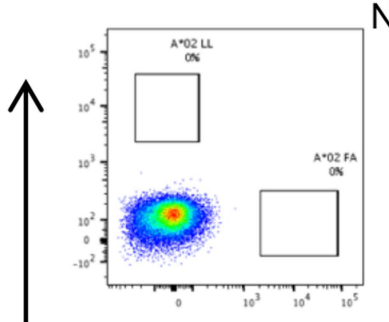

unstained

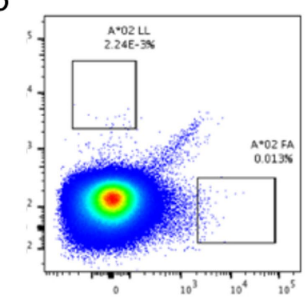

tetramer

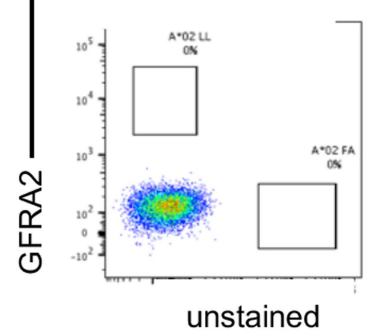

ND388

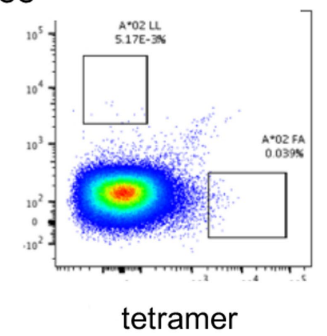

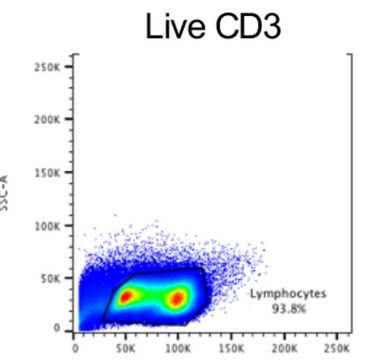

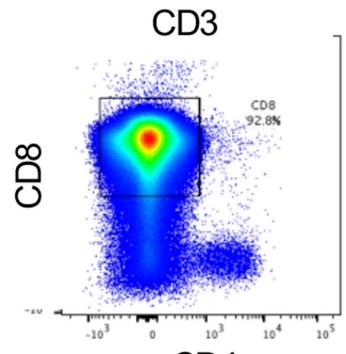

CD4

ND371
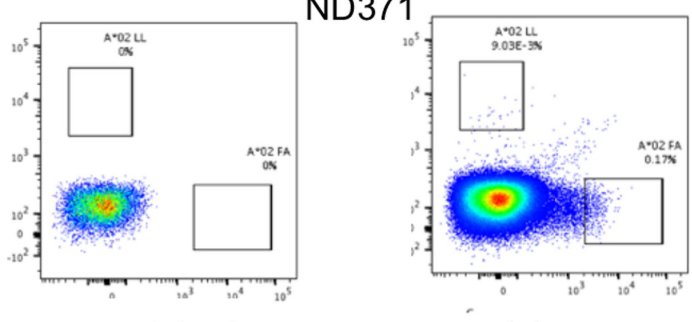

tetramer

IGFBPL1

\begin{tabular}{|c|c|c|c|c|c|c|c|}
\hline Donor: & A*02 LL & $A * 02 F A$ & LL binders & FA binders & $\begin{array}{c}\text { Geometric } \\
\text { mean LL }\end{array}$ & $\begin{array}{c}\text { Geometric } \\
\text { mean FA }\end{array}$ & \multirow{4}{*}{$\begin{array}{l}\text { LL9: } \\
\text { IGFBPL1 } \\
\text { FA10: } \\
\text { GFRA2 }\end{array}$} \\
\hline ND046 & $0.0016 \%$ & $0.0089 \%$ & 358 & 2051 & 3418 & 3902 & \\
\hline ND371 & $0.0064 \%$ & $0.1200 \%$ & 1150 & 21600 & 4796 & 4568 & \\
\hline ND388 & $0.0027 \%$ & $0.0210 \%$ & 625 & 4830 & 4157 & 4347 & \\
\hline ND436 & $0.0140 \%$ & $0.0740 \%$ & 6720 & 35520 & 3896 & 4410 & \\
\hline
\end{tabular}

d

MART-1

(+ control)

PHOX2B

$\mathrm{TH}$

IGFBPL1

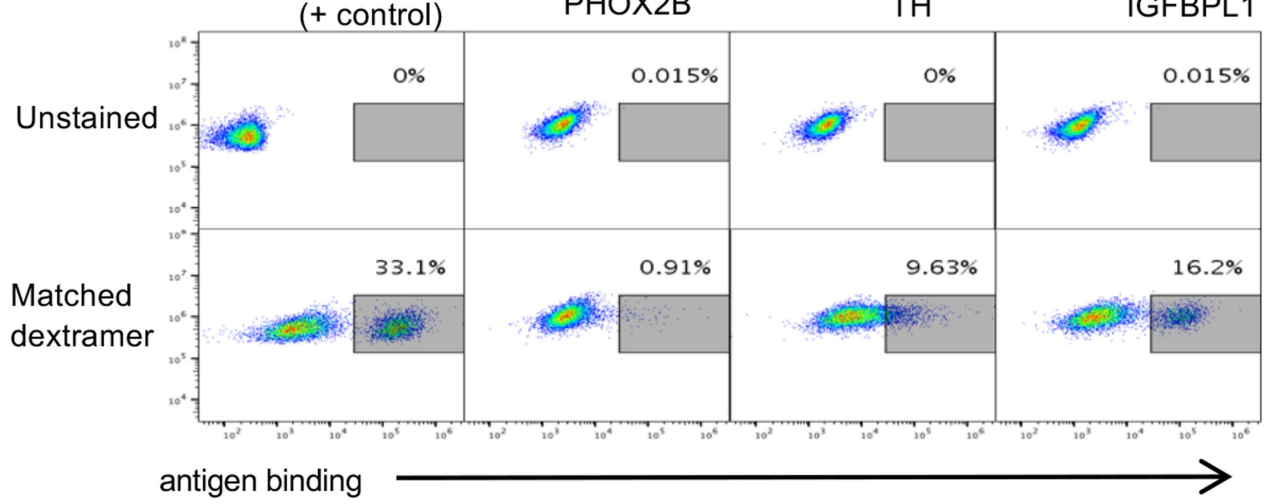

Extended Data Fig. 8 | Detection of tumor self-antigen specific CD8 T cells in normal donors. a. Gating used to select CD8 T cells. Similar gating strategy was used to select live singlets for Jurkat and primary cells transduced with CAR constructs (without selection for CD3 and exclusion of CD14, CD19, and CD4). b. Four normal donors stained with IGFBPL1 dextramer on $\mathrm{x}$-axis and GFRA2 on $y$-axis shows rare population of antigen-specific cells varying by donor and antigen (left panel showing unstained and right panel showing tetramer stained).c. Frequencies and MFIs of antigen specific cells across donors. d. Top constructs generated from normal donor-derived antigen-specific TCRs found by single-cell sequencing show range of antigen binding PHOX2B, TH, and IGFBPL1 as compared to DMF5 receptor targeting MART-1. Screening for antigen specific $T$ cells in 3 donors reveals that PHOX2B TCRs have minimal target binding, suggesting that $\mathrm{PHOX} 2 \mathrm{~B}$ self-antigen is immunogenically silent and warrants targeting using synthetic scFv receptors. 
a

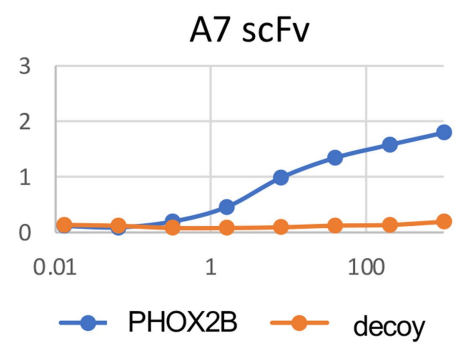

C

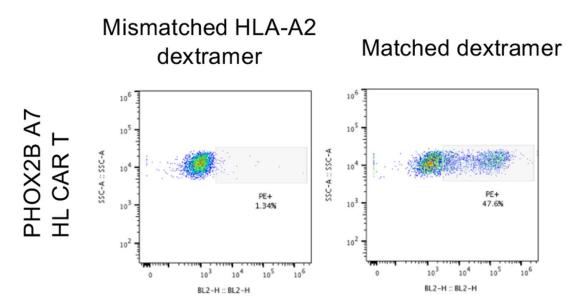

\section{f}
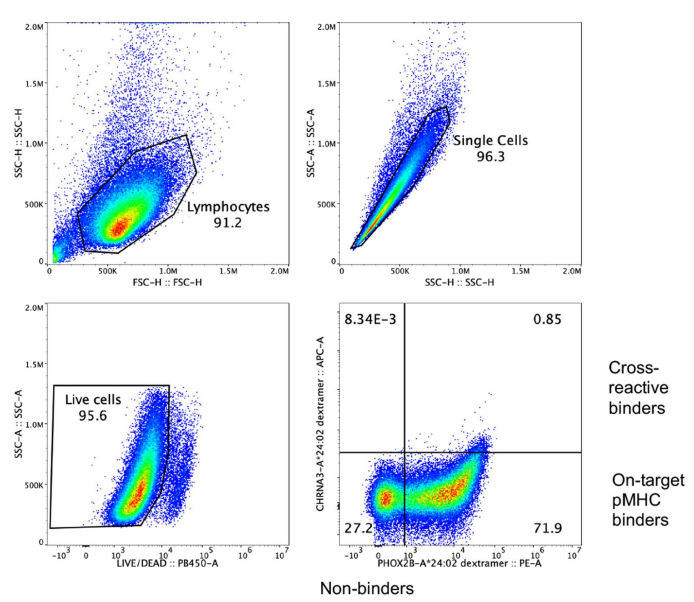

Extended Data Fig. 9 | Development of antigen-specific CARs for neuroblastoma antigens. a. ELISA of $\mathrm{PHOX} 2 \mathrm{~B} \mathrm{scFv} A 7 \mathrm{using} \mathrm{PHOX} 2 \mathrm{~B} \mathrm{p} / \mathrm{MHC}$ and decoy peptide on HLA-A*24:02. b. Schematic of second-generation CAR constructs. c. A7 CAR transduced into primary CD8 cells binds PHOX2B dextramer but not HLA-A*02:01 dextramer. d. A7 CAR preferentially binds
PHOX2B dextramer but cross-reacts with mismatched peptides $P B K$ and CHRNA3 on HLA-A*24:02 at high affinity. e. A7 CAR potently kills HLA-A*24:02 neuroblastoma lines, but also kills $\mathrm{HLA}^{+} /$antigen $^{-}$tumor cells.f. Tetramer and dextramer gating strategy for pMHC staining. 


\section{Article}

a SKNAS + non-specific CAR
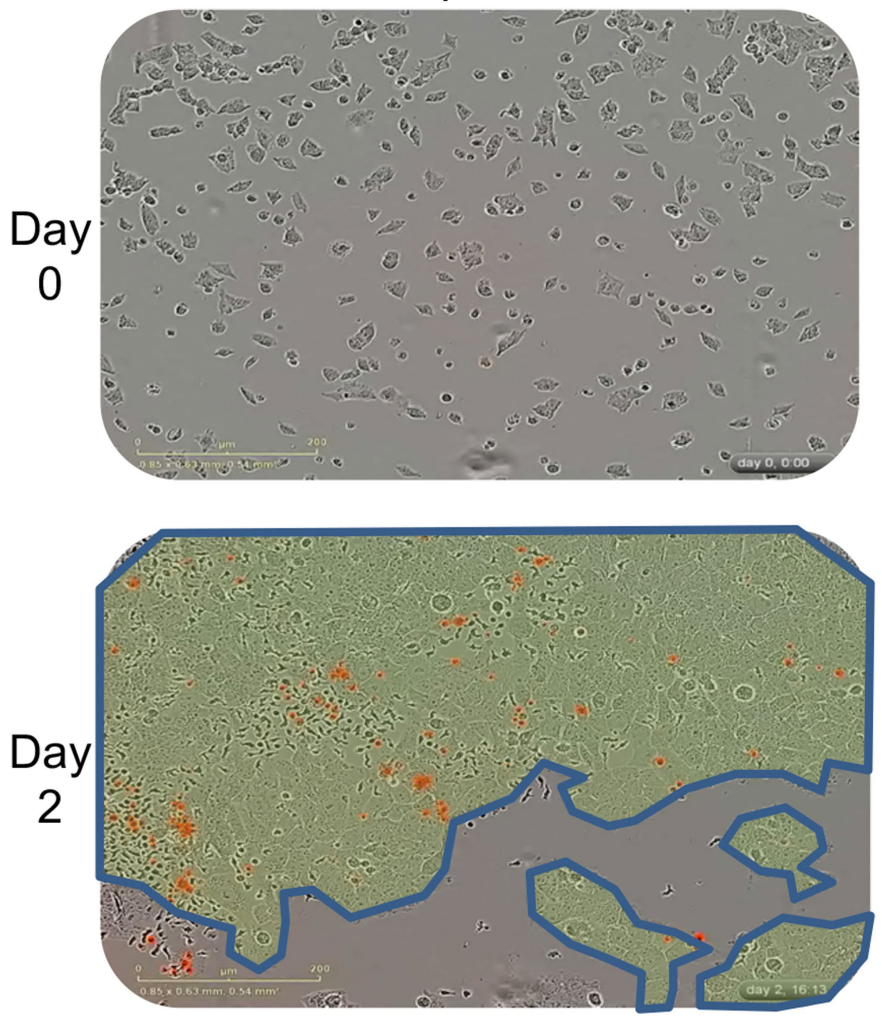

Tumor cells

\section{SKNAS + anti PHOX2B A7 CAR}
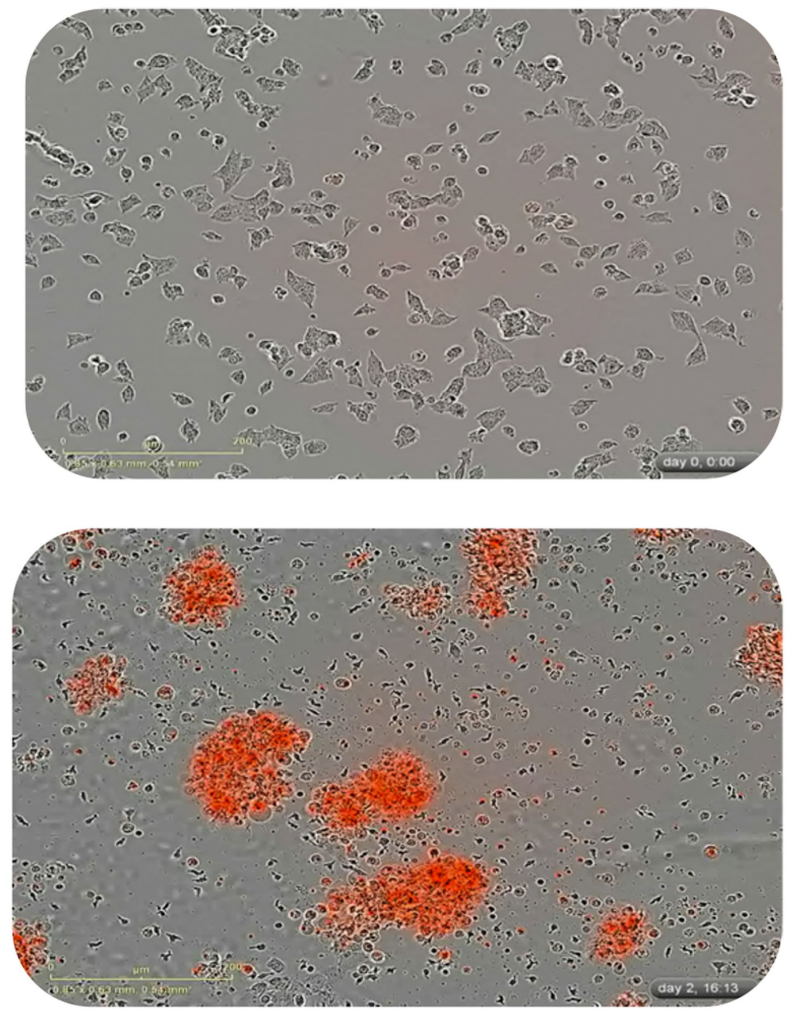

Cleaved caspase

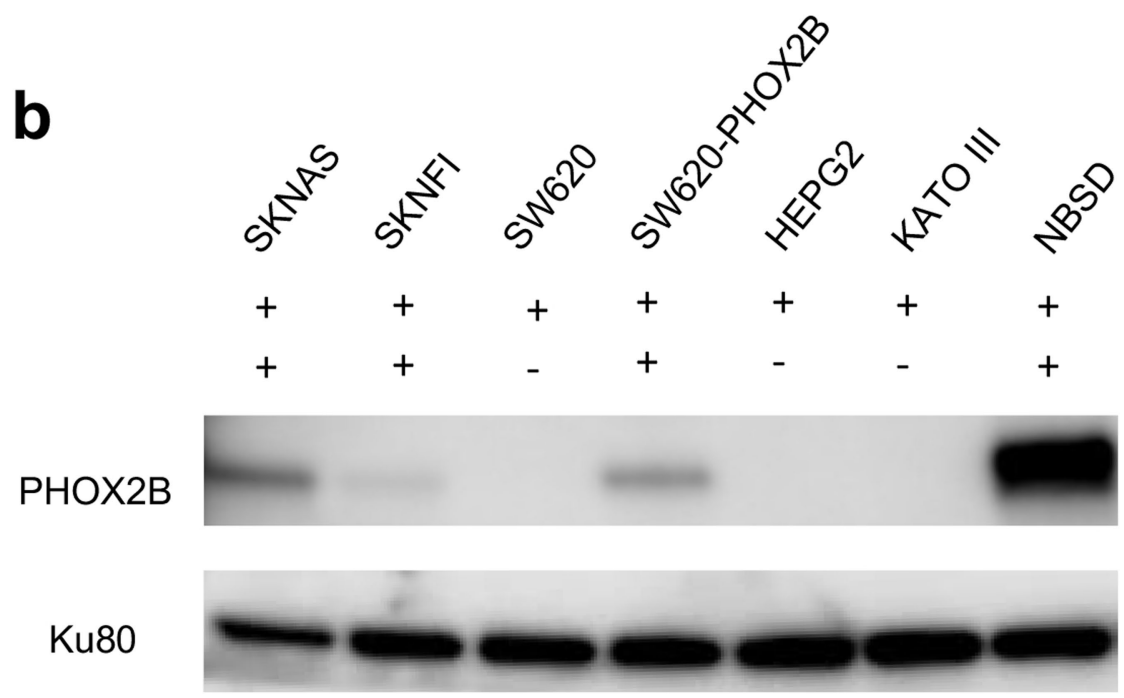

Extended Data Fig. 10 | PHOX2B A7 CAR. a. SKNAS tumor cells co-cultured with A7 CAR shows potent killing. SKNAS cells plated on day 0 (top), nontargeting CAR (left) and A7 CAR (right) added after $18 \mathrm{~h}$; measuring tumor confluence (green) and cleaved caspase (red) on day 2 (bottom) shows tumor outgrowth with non-specific CAR and killing of all tumor cells with A7 CAR. Representative images shown from experiment repeated three times using $\mathrm{n}=3$ replicates in each experiment. b. PHOX2B is expressed in neuroblastoma cell lines (SKNAS, SKNFI, and NBSD), and not in HLA-matched controls (SW620, KATO III, and HEPG2). PHOX2B is expressed in SW620 cells transduced with full-length $\mathrm{PHOX} 2 \mathrm{~B}$. For gel source data from single experiment, see Supplementary Fig. 1. 
a

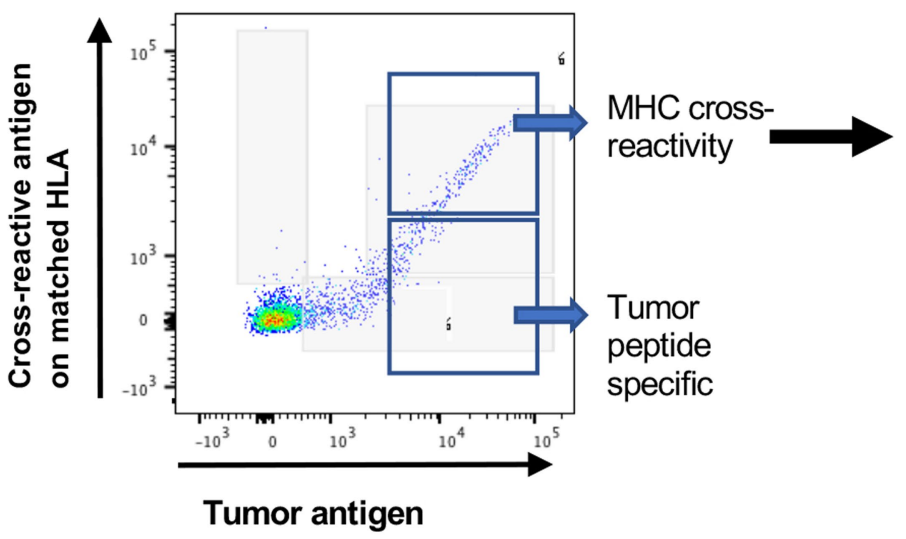

b

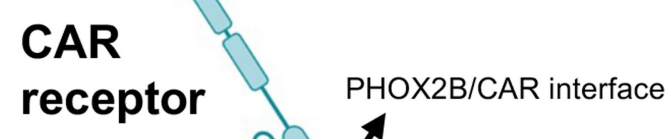

$\mathrm{MHC} / \mathrm{CAR}$

interface

MHC

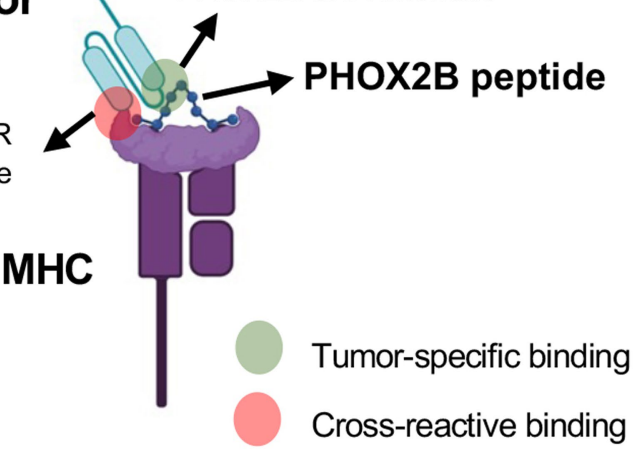

WT A7 CAR

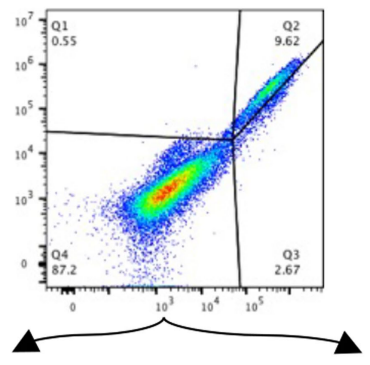

A7 D3-3 mut

A7 R3-2 mut

\begin{tabular}{|l|r|}
\hline $2-3$ & 1.17 \\
\hline $2-4$ & 0.89 \\
\hline $2-5$ & 1.17 \\
\hline $2-6$ & 0.65 \\
\hline $2-7$ & 1.00 \\
\hline $3-1$ & 0.90 \\
\hline $3-2$ & 4.71 \\
\hline $3-3$ & 12.40 \\
\hline $3-4$ & 3.39 \\
\hline $3-5$ & 30.51 \\
\hline $3-6$ & 8.70 \\
\hline $3-7$ & 1.69 \\
\hline $3-8$ & 4.53 \\
\hline $3-9$ & 1.38 \\
\hline $3-10$ & 10.00 \\
\hline $3-11$ & 1.07 \\
\hline
\end{tabular}

Contribution to $\mathrm{MHC}$ binding

Extended Data Fig. 11 | Saturation mutagenesis of A7 construct resulted in single-antigen-specific population. a-b. Cross-reactive binding to mismatched HLA-A24 peptides. Flow cy tometry of Jurkat cells transduced with A7 CAR stained with $\mathrm{PHOX} 2 \mathrm{~B}$ dextramer on $\mathrm{x}$-axis and mismatched $\mathrm{PBK}$ peptide on HLA-A*24:02 on y-axis.c. Saturation mutagenesis was performed on CDR loops 1-3 of the heavy chain. Each pool of mutants was stained with target pMHC and counter-stained with mismatched pMHC. Contribution of each CDR binding loop (mutagens labeled CDR loop \# - position \#) to binding HLA-A*24:02 is shown (green: no contribution to binding; red: significant HLA binding at specific amino acid). Contribution of each position to HLA binding was calculated as follows: $\left(\mathrm{MFI}_{\text {target(mut) }} / \mathrm{MFI}_{\text {mismatch(mut })}\right) /\left(\left(\mathrm{MFI}_{\text {target(WT })} / \mathrm{MFI}_{\text {mismatch(WT)} \text {. }}\right.\right.$ d. Mutations of A7 CAR at CDR3 positions 2 and 3 result in $12.4 x$ and $4.7 x$ shifts towards single specificity, respectively. Double mutation of D3A and R2A resulted in modified A7 CAR with no MHC cross-reactivity and reduced binding to target. Created with BioRender.com. 


\section{Article}

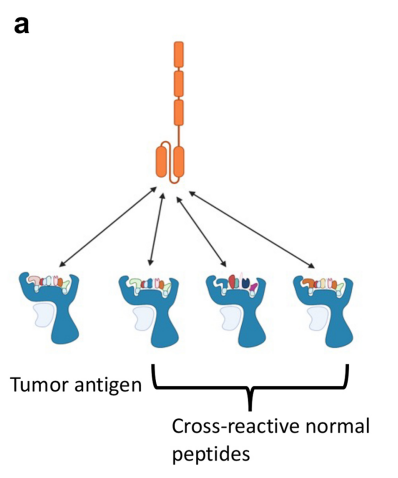

b
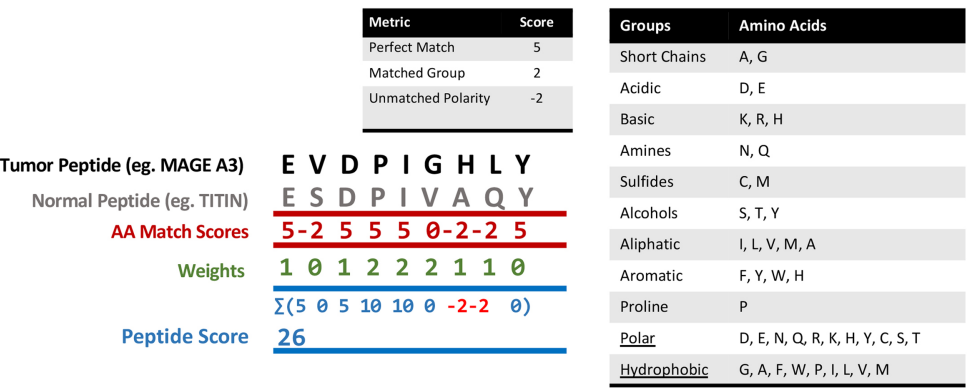

c

\begin{tabular}{|c|c|}
\hline & 84 HLAs \\
\hline $\begin{array}{l}20,394 \\
\text { human } \\
\text { proteins }\end{array}$ & $\begin{array}{l}92.4 \times 10^{6} \\
\text { normal MHC } \\
\text { peptides }\end{array}$ \\
\hline
\end{tabular}

Tumor Peptide protein peptides

EVDPIGHLY
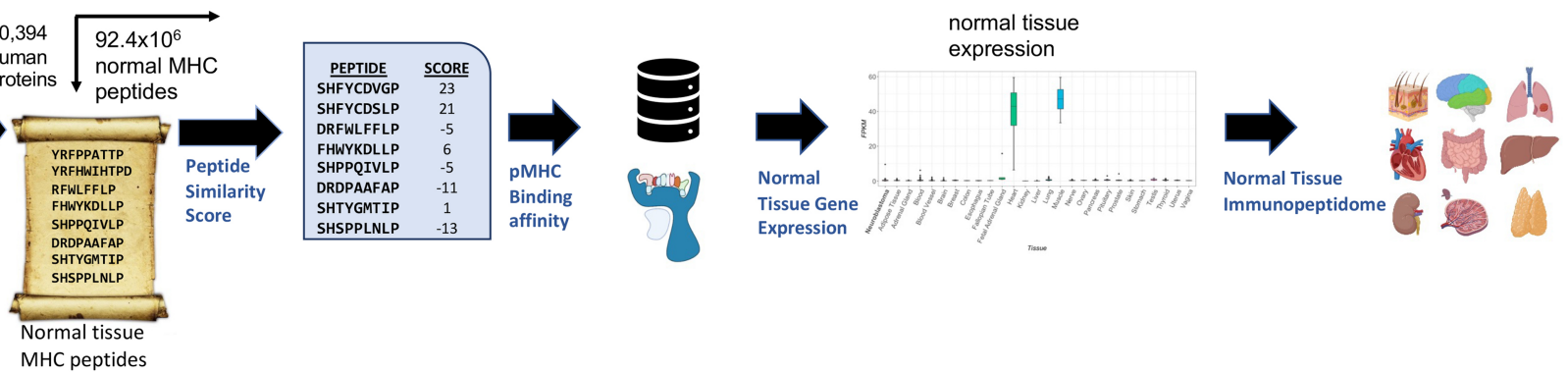

\section{Tissue Gene} name

be presented from the normal proteome (totaling $92.4 \times 10^{6}$ potential $\mathrm{MHC}$

Extended Data Fig. 12 | sCRAP cross-reactivity algorithm. a. Cross-reactivity algorithm was developed to identify peptides presented on normal tissue with similar biophysical properties to tumor antigens such as to pre-emptively predict cross-reactivities and screen for specificity. b. Illustration of peptide scoring system described in methods. c. Schematic of algorithm workflow peptides). Binding affinity is predicted for each normal peptide and maximum gene expression of parent gene are factored into the overall score of each peptide. Peptides are referenced against a normal tissue immunopeptidomics describing how tumor peptides are scored against each peptide predicted to database. Created with BioRender.com. 
a

\begin{tabular}{|c|c|c|c|c|c|c|c|}
\hline Rank & Peptide_Score & Peptides & Gene_Name & Max_norm & Max_tissue & Binding & Overall_score \\
\hline & $\mathbf{5 0}$ & EVDPIGHLY & MAGEA3 & $\mathbf{8 . 8 4 9 1 2 7}$ & Testis & $\mathbf{1 1 . 4 3}$ & \\
\hline 1 & 25 & LSDPVGTCY & ITGA5 & 1238.931519 & Blood Vessel & 3.46 & 8235.7 \\
\hline 2 & 32 & YTDPVGVLY & LCAT & 105.2005 & Liver & 2.18 & 1447.7 \\
\hline 3 & 24 & FLEPLGLAY & LENG8 & 270.2617493 & Uterus & 6.87 & 944.1 \\
\hline 4 & 26 & ESDPIVAQY & TTN & 261.3758 & Muscle & $\mathbf{8 . 0 7}$ & 842.1 \\
\hline 5 & 25 & YIDPIAMEY & LAT2 & 141.4519 & Blood & 5.7 & 620.4 \\
\hline 6 & 25 & LQDPLGLAY & RGS14 & 401.9189 & Brain & 20.36 & 493.5 \\
\hline 7 & 25 & EMDPVTQLY & ALCAM & 110.1399 & Skin & 5.68 & 484.8 \\
\hline 8 & 25 & CTFPLGWLY & ELOVL5 & 391.8979492 & Breast & 27.21 & 360.1 \\
\hline 9 & 28 & VSDPVGVLY & JKAMP & 43.49138 & Brain & 3.91 & 311.4 \\
\hline 10 & 24 & QLDGLGFLY & ACSBG2 & 227.0139 & Testis & 22.79 & 239.1 \\
\hline 11 & 26 & SLDPLAMLY & TSHZ1 & 34.86856 & Ovary & 6.23 & 145.5 \\
\hline 12 & 24 & GTDPAADLY & DNAH1 & 18.27267 & Testis & 4.7 & 93.3 \\
\hline 13 & 23 & DSDPTGTAY & SUOX & 26.187 & Kidney & 6.62 & 90.9 \\
\hline 14 & 30 & PLDPLGHNY & CILP & 238.8102 & Adipose Tissue & 96.56 & 74.2 \\
\hline
\end{tabular}

\section{$1,143,861$ peptides}

b

\section{TITIN normal tissue expression}

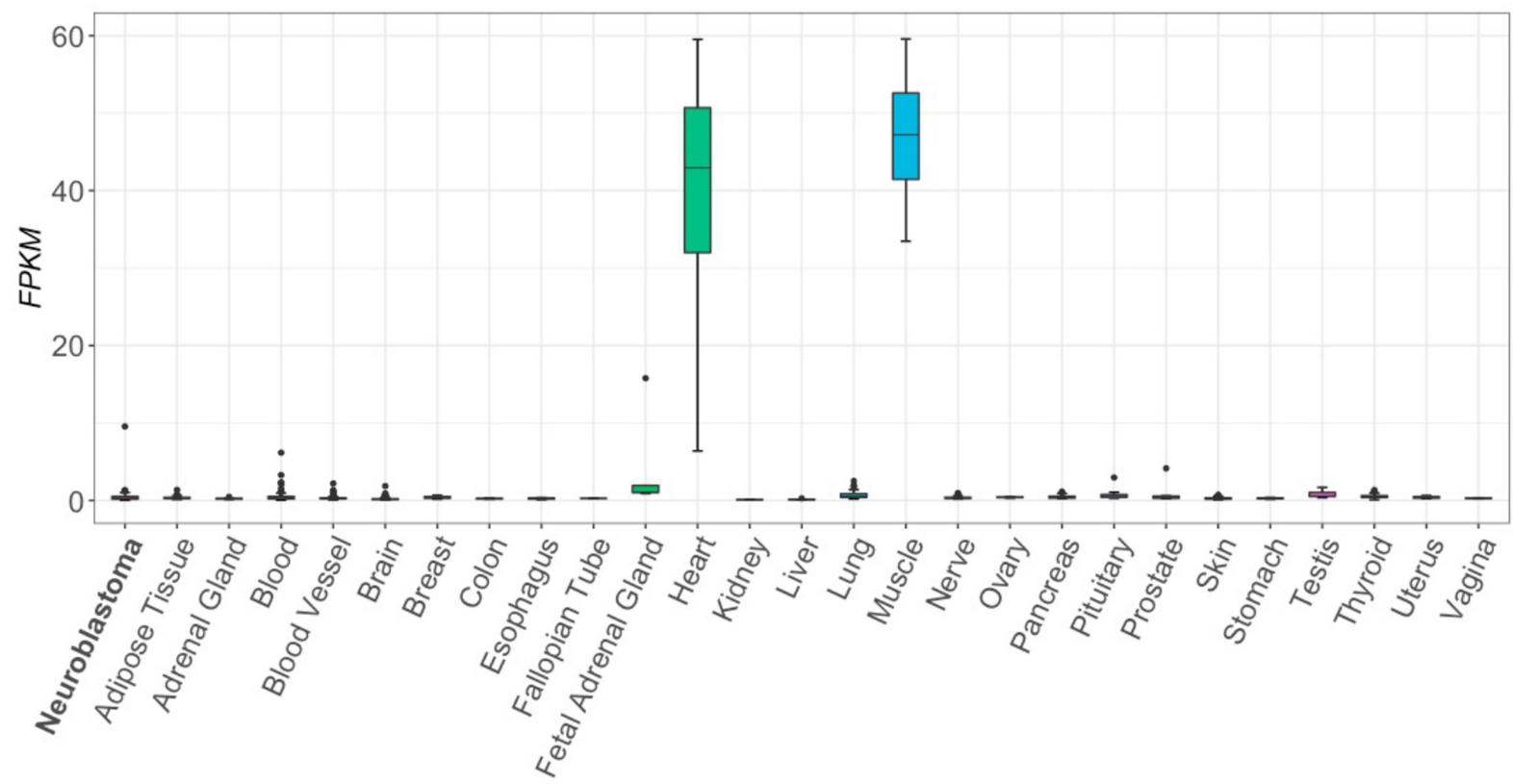

Tissue

Extended Data Fig. 13 | pMHC Cross-Reactivity Algorithm sCRAP Predicts MAGE-A3 toxicity through TITIN. a. Table of top predicted cross-reactive peptides to MAGE-A3 peptide EVDPIGHLY reveals cross-reactivity with Titin peptide ESDPIVAQY ranks $4^{\text {th }}$ out of $1,143,861$ potential peptides presented on HLA-A*01:01.b. TITIN is highly expressed in GTEx RNA-sequencing of heart $(n=108)$ and muscle $(n=138)$ tissues. 


\section{Article}

a

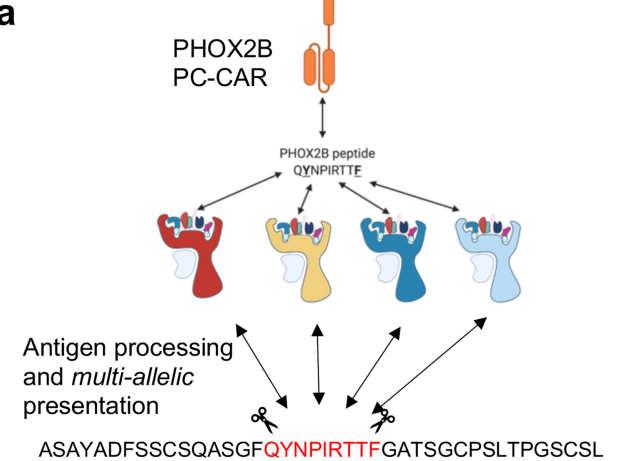

b

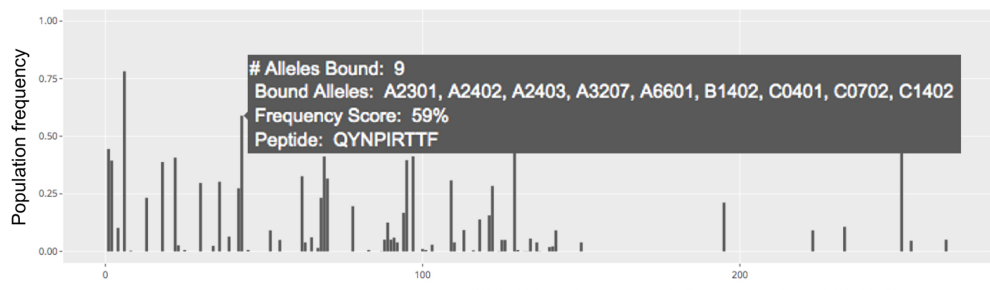

PHOX2B antigen potentially presented on 9 HLA allotypes Top predicted allotypes: HLA-A ${ }^{\star} 24: 03: 3.7 \mathrm{nM}(0.03 \%$ rank $)$

Discovered by immunopeptidomics on: $\longrightarrow \begin{aligned} & \text { HLA-A } 23: 01: 50.8 \mathrm{nM}(0.2 \% \text { rank }) \\ & \text { HLA-C*07:02: } 97.5 \mathrm{nM}(0.03 \% \text { rank })\end{aligned}$ HLA-C*14:02: $33.9 \mathrm{nM}(0.12 \%$ rank)

(up to $59 \%$ US population)

( $20 \%$ US population)
C

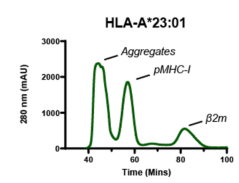

d f

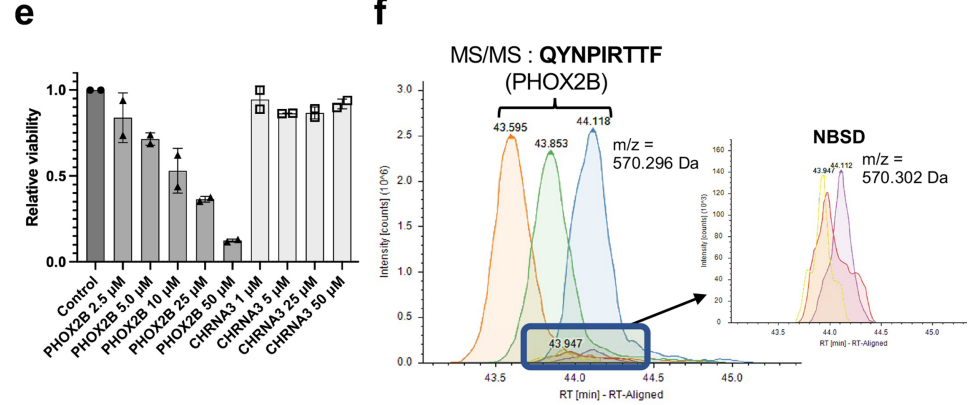

Extended Data Fig 14 | Cross-HLA recognition of PHOX2B peptide. a. Schematic of hypothesis proposing that $\mathrm{PHOX} 2 \mathrm{~B}$ peptide QYNPIRTTF detected by immunopeptidomics can be presented by additional $\mathrm{HLA}$ alleles after undergoing a common antigen processing pathway.b. Population-scale presentation across the length of the PHOX2B protein (all potential 9 mers on $x$-axis) generated by ShinyNAP ${ }^{8}$ predicts $P H O X 2 B$ peptide QYNPIRTTF to be presented by an additional 8 HLA alleles in addition to HLA-A*24:02. Additionally, QYNPIRTTF was found to bind additional common HLA alleles HLA-C*07:01, HLA-C*06:02, HLA-A*29:02, and HLA-A*32:01 using NetMHCpan $4.1^{65}$ and also predicted by HLAthen ${ }^{66}$. c. Size exclusion chromatography of PHOX2B peptide QYNPIRTTF refolded with HLA-A*23:01, HLA-B*14:0, and HLA-C*07:02 shows formation of stable pMHC complex with each allotype.d. PC-CAR 10LH binds PHOX2B on HLA-A*23:01 demonstrates higher binding than 302LH, in concordance with observed in vivo activity (Fig. 4g).e.10LHCAR kills HLA-A*23:01/PHOX2B ${ }^{-}$WM873 cells when pulsed with PHOX2B peptide but not with CHRNA3 peptide $n=2$ technical replicates; reported as mean + -SD. f. Matched peptide search identifies unfragmented peaks in additional neuroblastoma tumors (NBSD shown). Unfragmented NBSD peaks are within $0.006 \mathrm{Da} \mathrm{m} / \mathrm{z}$ of peaks in which PHOX2B peptide QYNPIRTTF was identified by MS/MS in other samples and eluted within one minute of fragmented peaks. While validated MS/MS peaks were found in 2/8 PDX tumors and 2/8 primary tumors, peaks with $\mathrm{m} / \mathrm{z}$ and retention times matched to validated QYNPIRTTF peaks were identified in $6 / 8$ PDX tumors and $7 / 8$ primary tumors. Created with BioRender.com. 
a

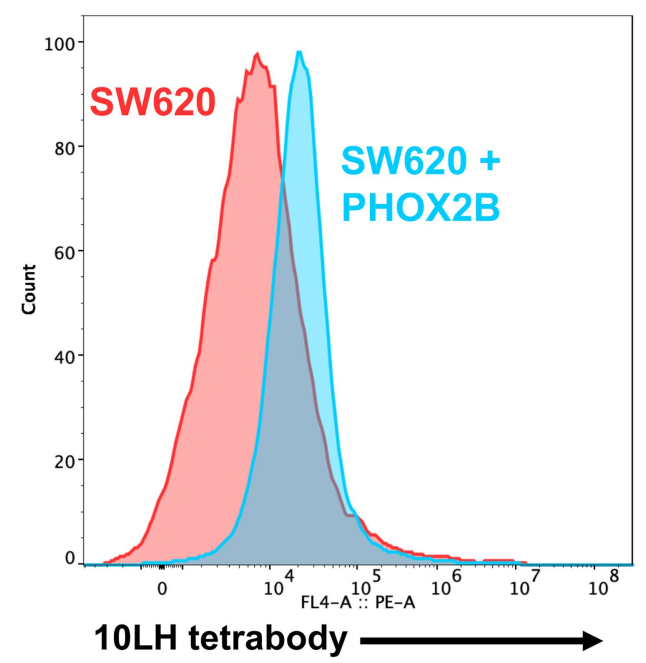

b

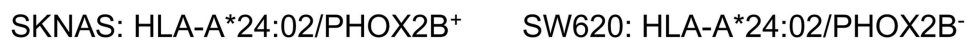

$320 \mathrm{LH}$
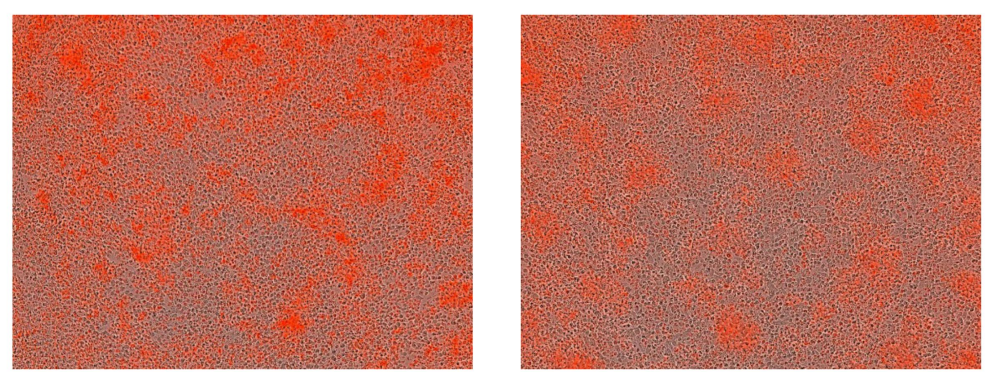

$280 \mathrm{LH}$

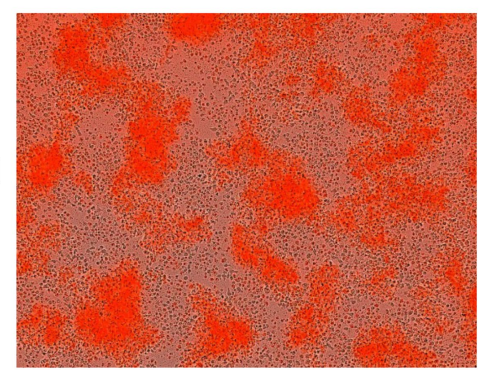

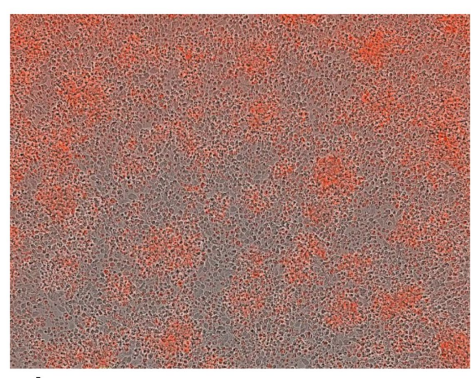

$400 \mu \mathrm{m}$
Extended Data Fig. $15 \mid$ a. Tetramerized 10LH binds to PHOX2B pMHC. Pulsing SW620 HLA-A*24:02 with 50 $\mu$ M PHOX2B peptide results in $10 \mathrm{LH}$ tetrabody binding. b. CARs predicted to be cross-reactive show killing in HLA-matched cells. CARs 320 LH and 280LH predicted to cross-react with peptides presented on normal tissue demonstrate significant cross-reactivity in HLA-matched SW620 cells. Representative images shown from $n=3$ technical replicates. 


\section{Article}

COG-564
untransduced T
cells

COG-564 10LH PC-CAR

\section{COG-564 302LH CAR}
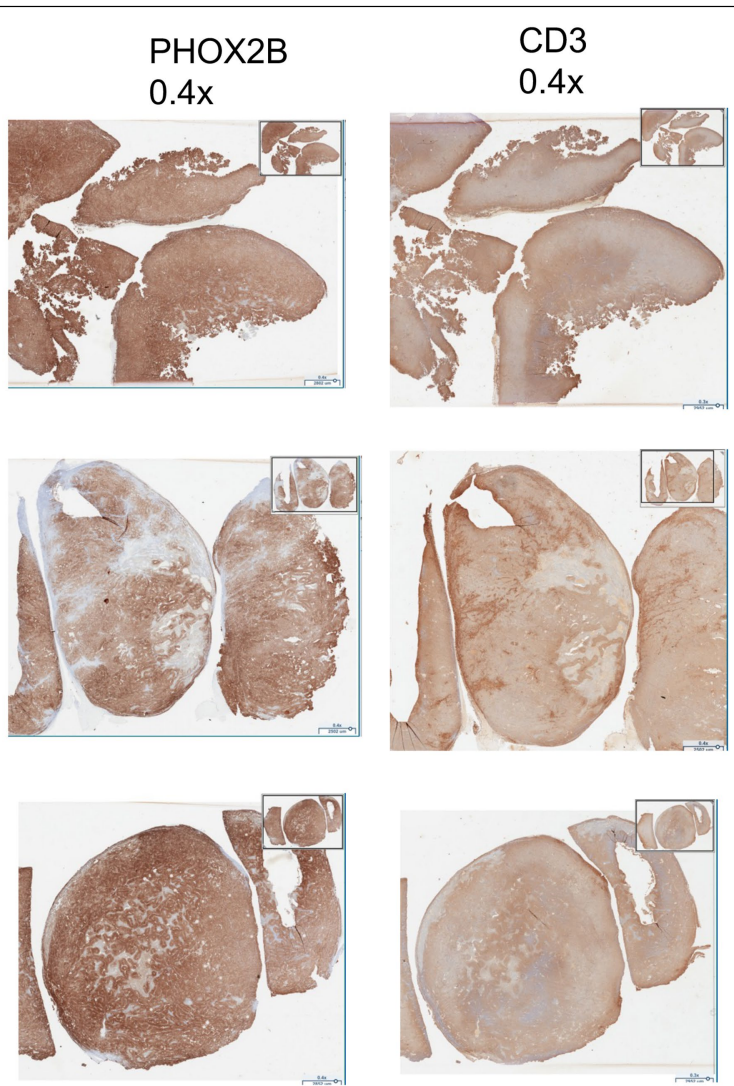

PHOX2B

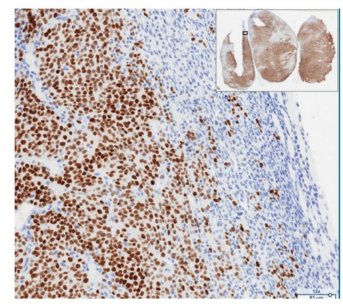

$10 \mathrm{x}$

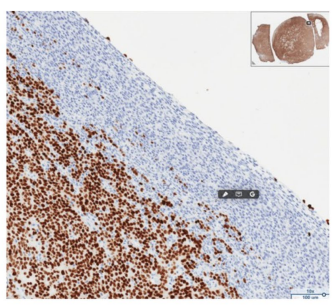

\section{CD3}
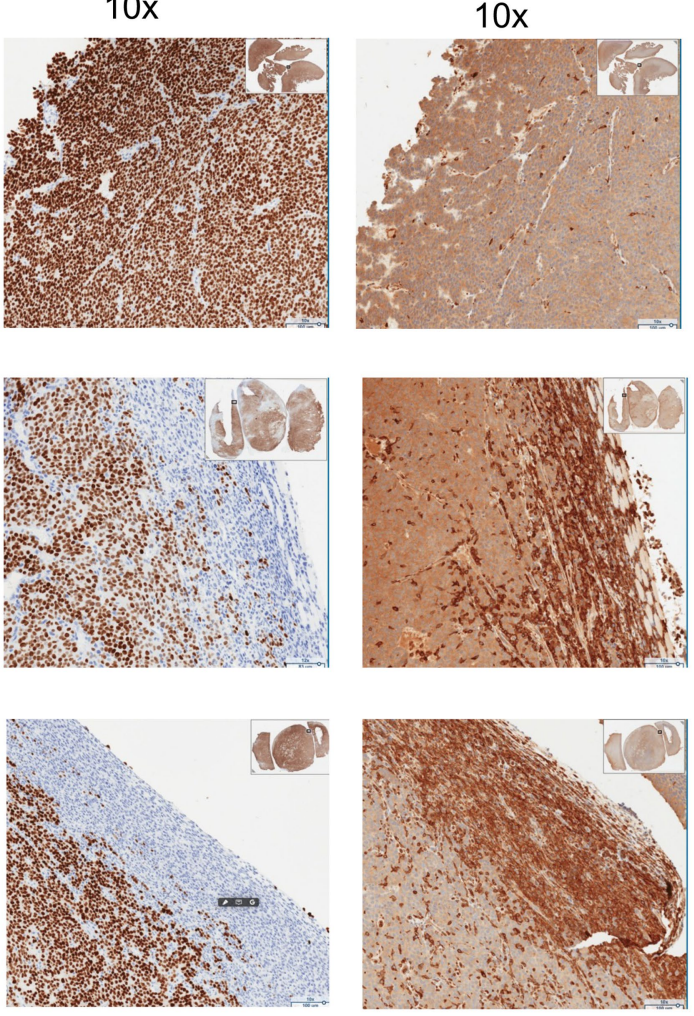

Extended Data Fig. 16 | Immunohistochemistry of tumors collected from mice exceeding tumor burden. COG-564x tumor-bearing mice treated with 10LH and 302LH PC-CARs show T cell infiltration as measured by CD3, co-localized with loss of PHOX2B target expression and tissue necrosis. No evidence of $\mathrm{T}$ cell infiltration observed in mice treated with untransduced
T cells. Tumors were collected from one of each mouse in treatment arms reaching tumor burden 11 days after receiving $T$ cells. All other mice in treatment arms went on to achieve complete responses. Images shown from tumors collected from lone mice reaching tumor burden each study arm in single experiment. 
a

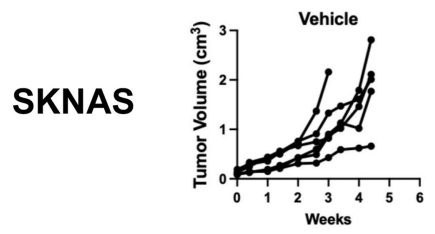

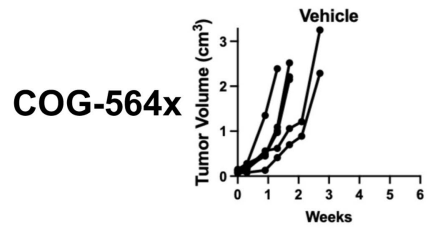

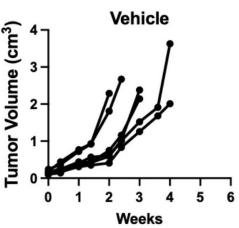

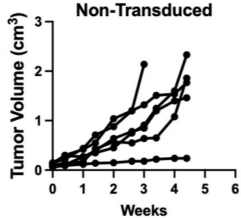

UT
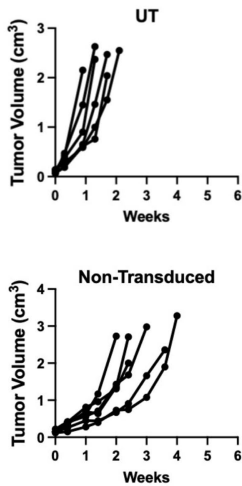
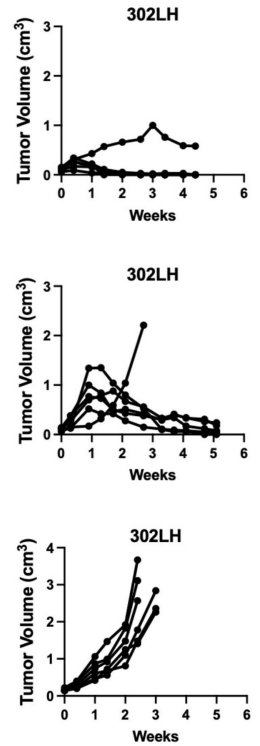

b
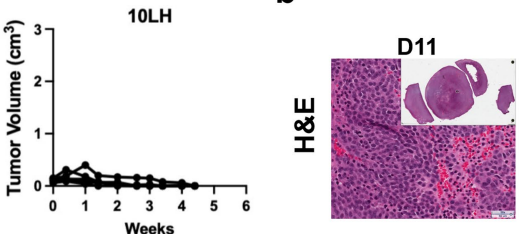

Endpoint (D36)
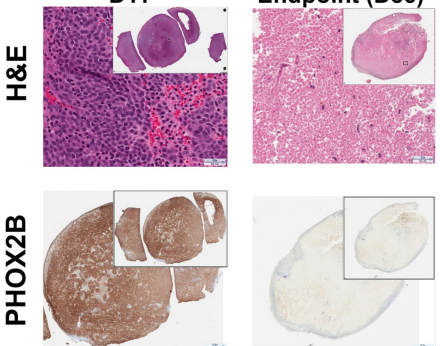
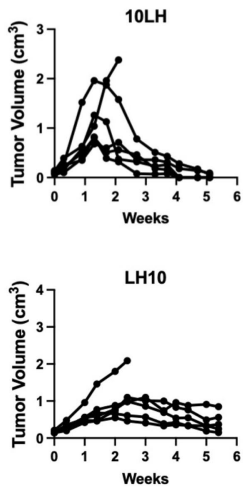

Extended Data Fig. 17 PC-CARs result in tumor ablation in mice engrafted with patient-derived xenografts. a. Tumor regression in individual mice in vivo treated with PC-CARs $(n=6$; source data provided in Supplementary Table 3).b.COG-564x tumors collected from 302LH PC-CAR-treated mice collected at when mice reached tumor burden on day 11 (left) and endpoint of study on day 38 (right). Though tumors are detectable at endpoint, H\&E and PHOX2B staining of 302LH PC-CAR-treated tumors reveals entirely necrotic tissue by endpoint of the study. Single available tumor collected from mouse reaching tumor burden for D11 and single endpoint tumor in one experiment collected for immunohistochemistry shown. 


\section{Article}

Extended Data Table 1 | Antigen discovery strategies in neuroblastoma

a

\begin{tabular}{|c|c|c|c|c|c|c|c|c|}
\hline Sample ID & Sample Type & Sex & Age & Timing of collection & Status & Stage & MYCN & Driver Mutations \\
\hline COG-N-415x & PDX & Female & 1 & Post-mortem & Dead & 4 & Amplified & ALK F1174L \\
\hline COG-N-440x & PDX & Female & 0 & Post-mortem & Dead & 4 & Amplified & None \\
\hline COG-N-471x & PDX & Female & 2 & Post-mortem & Dead & 4 & Amplified & None \\
\hline NB-1691 & CDX & Male & NA & Progressive Disease & Dead & 4 & Amplified & None \\
\hline NB-1771 & CDX & Male & NA & Diagnosis & Dead & 4 & Amplified & TP53 C135F \\
\hline NB-EBc1 & CDX & Male & 2 & Progressive Disease & Dead & 4 & Non-Amplified & KRAS G12D \\
\hline NB-SD & CDX & Male & NA & Progressive Disease & Dead & 4 & Amplified & ALK F1174L; TP53 C176F \\
\hline SKNAS & CDX & Female & 6 & Progressive Disease & Dead & 4 & Non-Amplified & TP53 H168R \\
\hline PALVKK & Tumor & Female & 4 & Diagnosis & Dead & 4 & Non-Amplified & No Data \\
\hline PANXJL & Tumor & Male & 15 & Diagnosis & Alive & 4 & Non-Amplified & ALKR1275Q \\
\hline PAPBJE & Tumor & Male & 5 & Diagnosis & Alive & 4 & Amplified & None \\
\hline PAPCTS & Tumor & Male & 5 & Diagnosis & Dead & 4 & Amplified & None \\
\hline PAPVRN & Tumor & Male & 6 & Diagnosis & Alive & 4 & Non-Amplified & PTPN11 Y197* \\
\hline PARKGJ & Tumor & Male & 4 & Diagnosis & Dead & 4 & Non-Amplified & PTPN11 Y197*; NF1 E470* \\
\hline PARZCJ & Tumor & Male & 0 & Diagnosis & Alive & 3 & Non-Amplified & None detected by RNA-seq \\
\hline PASEGA & Tumor & Male & 2 & Diagnosis & Dead & 4 & Non-Amplified & None \\
\hline
\end{tabular}

b

\begin{tabular}{|l|l|l|}
\hline Gene & Peptide & Confidence \\
\hline PHOX2B & QYNPIRTTF & Validated \\
\hline MYCN & KATEYVHSL & High \\
\hline GATA3 & HNINRPLTM & High \\
\hline TBX2 & YQNDKITQL & High \\
\hline TBX2 & AQPSFFPAL & High \\
\hline TBX2 & AAAEAGLHV & High \\
\hline ASCL1 & RNRVKLVNLGF & Needs validation \\
\hline HAND2 & KELNEILKST & Needs validation \\
\hline ISL1 & GNQIHDQY & Needs validation \\
\hline
\end{tabular}

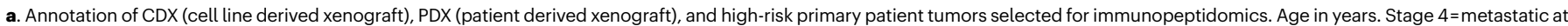

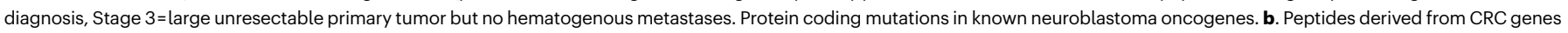

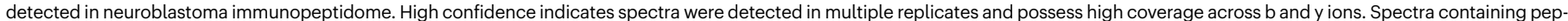

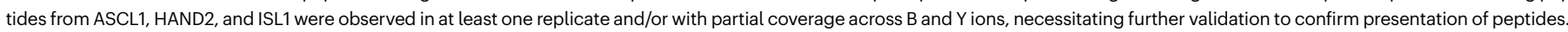




\section{Reporting Summary}

Nature Portfolio wishes to improve the reproducibility of the work that we publish. This form provides structure for consistency and transparency in reporting. For further information on Nature Portfolio policies, see our Editorial Policies and the Editorial Policy Checklist.

\section{Statistics}

For all statistical analyses, confirm that the following items are present in the figure legend, table legend, main text, or Methods section.

n/a Confirmed

$\square$ The exact sample size $(n)$ for each experimental group/condition, given as a discrete number and unit of measurement

$\square$ \ A statement on whether measurements were taken from distinct samples or whether the same sample was measured repeatedly

$\square$ The statistical test(s) used AND whether they are one- or two-sided

$\square$ Only common tests should be described solely by name; describe more complex techniques in the Methods section.

$\square$ \A description of all covariates tested

Х $\square$ A description of any assumptions or corrections, such as tests of normality and adjustment for multiple comparisons

$\square$ A full description of the statistical parameters including central tendency (e.g. means) or other basic estimates (e.g. regression coefficient)

$\triangle$ AND variation (e.g. standard deviation) or associated estimates of uncertainty (e.g. confidence intervals)

$\varnothing$ For null hypothesis testing, the test statistic (e.g. $F, t, r$ ) with confidence intervals, effect sizes, degrees of freedom and $P$ value noted

Give $P$ values as exact values whenever suitable.

Х $\square$ For Bayesian analysis, information on the choice of priors and Markov chain Monte Carlo settings

Х $\square$ For hierarchical and complex designs, identification of the appropriate level for tests and full reporting of outcomes

\ $\square$ Estimates of effect sizes (e.g. Cohen's $d$, Pearson's $r$ ), indicating how they were calculated

Our web collection on statistics for biologists contains articles on many of the points above.

\section{Software and code}

Policy information about availability of computer code

Data collection Flow cytometry data was collected using CytExpert (Beckman Coulter) and FACSDiva ( v8, BD Biosciences). FACSAria Fusion (BD Biosciences) was used for cell sorting. 10x Genomics 5' V(D)J Kits were used on the Chromium machine and sequenced using the lllumina MiSeq. Orbitrap Fusion Lumos (Thermo Fisher Scientific) was used for mass spectrometry. BLItzTM system (ForteBio, USA) was used for binding assays. Incucyte ZOOM and S3 (Essence BioScience) was used in T cell cytotoxicity assays. Aperio CS-O slide scanner (Leica Biosystems) was used in scanning IHC slides. Sanger sequencing was performed at CHOP NapCore.

Data analysis Flow cytometry analysis was performed using FlowJo (v10.7.I, BD Biosciences), R Studio, and Microsoft Excel were used to analyze data. Cellranger VDJ was used to analyze single-cell TCR data. SequestHT algorithm in the Proteome Discoverer (v2.1 and v2.4, ThermoFisher) software was used for LC/MS/MS analysis. NetMHC-4.0 was used in PMHC binding predictions. PH LAT 1.1 was used for HLA typing. Blitz Pro TM software was used to analyze scFv binding data. PyMOL v2.4.1 and RosettaMHC were used for structural modeling. DNA constructs and sequencing data analyzed using SnapGene v5.2 and Benchling. Gene Ontology analyses were performed using PANTHER (v16.0).

Crystallization model building and refinement were performed using COOT and Phenix (v1.19.2), respectively. Our algorithms ShinyNAP and

SCRAP are described in methods and referenced, and have been made available to reviewers at: https://marisshiny.research.chop.edu/sCRAP/

For manuscripts utilizing custom algorithms or software that are central to the research but not yet described in published literature, software must be made available to editors and reviewers. We strongly encourage code deposition in a community repository (e.g. GitHub). See the Nature Portfolio guidelines for submitting code \& software for further information. 
Policy information about availability of data

All manuscripts must include a data availability statement. This statement should provide the following information, where applicable:

- Accession codes, unique identifiers, or web links for publicly available datasets

- A description of any restrictions on data availability

- For clinical datasets or third party data, please ensure that the statement adheres to our policy

- Proteomics data is available on PRIDE database as follows:

Project Name: Neuroblastoma HLA class I Immunopeptidomics

Project accession: PXD027182

Project DOI: 10.6019/PXD027182

Reviewer username: reviewer_pxd027182@ebi.ac.uk

Reviewer password: JHiSKkIT

All proteins structures are available in the Protein Data Bank under accession codes HLA-A*02:01/LLLPLLPPL (PDB: 7MJ6), HLA-A*02:01/LLPLLPPLSP (PDB: 7MJ7),

HLA-A*02:01/LLPLLPPLSPS (PDB: 7MJ8), HLA-A*02:01/LLPRLPPL (PDB: 7MJ9), and HLA-A*24:02/QYNPIRTTF (PDB: 7MJA).

\section{Field-specific reporting}

Please select the one below that is the best fit for your research. If you are not sure, read the appropriate sections before making your selection. $\bigotimes$ Life sciences $\square$ Behavioural \& social sciences Ecological, evolutionary \& environmental sciences

For a reference copy of the document with all sections, see nature.com/documents/nr-reporting-summary-flat.pdf

\section{Life sciences study design}

All studies must disclose on these points even when the disclosure is negative.

Sample size Sample size for murine studies is based on our review of preclinical trials and publication 10.1158/0008-5472.CAN-16-0122 that show that $\mathrm{N}=6 / \mathrm{arm}$ is an ethically sound and statistically rigorous sample size for preclinical therapeutic trials.

Cytotoxicity and cytokine assays were performed using T cells from $n=3$ independent donors, a sample size that allows for statistically significant differences to be ascertained within the context of donor variability.

Data exclusions No data were excluded.

Replication In-vivo studies were conducted twice in independent experiments with similar results.

All mass spec samples were run in triplicate with similar conclusions, and all binding assays were performed in triplicate with matching results. All T cell assays were performed using three individual donors undergoing separate lentiviral transductions, and cytotoxicity and cytokine assays were performed with at least three biological and three technical replicates for all reported experiments with similar results between individual donors. Other in-vitro assays were typically reproduced at least three times in independent experiments.

Randomization Randomization of samples for the repeated studies was performed by selecting tumor sizes within a range of $0.06 \mathrm{~cm}^{\wedge} 3-0.19 \mathrm{~cm} \mathrm{~cm}^{\wedge} \mathrm{for} S K N A S$ and COG-N-564x. Using Excel, the tumors were selected and placed into $n=6$ groups to generate a median size of $0.11 \mathrm{~cm}^{\wedge} 3-0.12 \mathrm{~cm}^{\wedge} 3$ for COG-N-564x and SKNAS. A range of $0.12 \mathrm{~cm}^{\wedge} 3-0.24 \mathrm{~cm}^{\wedge} 3$ was selected for NBSD to generate a median size of $0.14 \mathrm{~cm}^{\wedge} 3-0.15 \mathrm{~cm}^{\wedge} 3$.

Blinding During in-vivo studies, blinding was performed during tumor measurement. For other experiments (including in-vitro experiments), blinding could not be completed due to lack of personnel necessary for facilitating adequate blinding. Data analysis was completed with familiarity of experimental conditions and groups.

\section{Reporting for specific materials, systems and methods}

We require information from authors about some types of materials, experimental systems and methods used in many studies. Here, indicate whether each material, system or method listed is relevant to your study. If you are not sure if a list item applies to your research, read the appropriate section before selecting a response. 
Materials \& experimental systems

\begin{tabular}{l|l}
\hline$n / a$ & Involved in the study \\
$\square$ & $\bigotimes$ Antibodies \\
$\square$ Eukaryotic cell lines \\
$\square$ Palaeontology and archaeology \\
$\square$ & $\square$ Animals and other organisms \\
$\square$ & $\square$ Clinical data
\end{tabular}

Methods

\begin{tabular}{l|l}
\hline n/a & Involved in the study \\
$\square$ & $\bigotimes$ ChIP-seq \\
$\square$ & $\bigotimes$ Flow cytometry \\
$\square$ & $\square$ MRI-based neuroimaging
\end{tabular}

\section{Antibodies}

Antibodies used

The following fluorophore-conjugated antibodies were used ("h" prefix refers to anti-human): hCD3 (Clone F7.2.38, Dako, A0452, 1:100), hPHOX2B (Clone EPR14423, Abcam, ab183741, 1:500), hHLA-ABC (Clone W6/32, Abcam, ab70328, 1:1200), FITC antiinfluenza A virus nucleoprotein (Clone D67J Abcam, ab210526, 1:100), APC hCD137 (4-1BB) (Clone 4B4-1, Biolegend, 309809, 1:100), FITC hCD3 (UCHT1, Biolegend, 300405, 1:100), Brilliant Violet 510 hCD19 (HIB19, Biolegend, 302241, 1:100), Brilliant Violet 605 hCD8a (RPA-T8, Biolegend, 301039, 1:100), PE-Cyanine5.5 hCD4 (S3.5, Invitrogen, MHCD0418, 1:100).

Validation
All antibodies used in immunohistochemistry, flow cytometry, and immunofluorescence were titrated and validated for intended use. Antibodies used for flow cytometry were compared to unstained and isotype controls, when applicable.
Manufacturer validation statements can be found below for the corresponding antibodies.
hCD3: https://www.agilent.com/en/product/immunohistochemistry/antibodies-controls/primary-antibodies/cd3-
(concentrate)-76649
hPHOX2B: https://www.abcam.com/phox2b-antibody-epr14423-c-terminal-ab183741.html
hHLA-ABC: https://www.abcam.com/hla-class-1-abc-antibody-emr8-5-ab70328.html
FITC anti-influenza A virus nucleoprotein: https://www.abcam.com/fitc-influenza-a-virus-nucleoprotein-antibody-d67j- ab210526.html
APC hCD137: https://www.biolegend.com/en-us/products/apc-anti-human-cd137-4-1bb-antibody-3910?Group/D=BLG2623
FITC hCD3: https://www.biolegend.com/en-us/products/fitc-anti-human-cd3-antibody-863
Brilliant Violet 510 hCD19: https://www.biolegend.com/en-us/products/brilliant-violet-510-anti-human-cd19-antibody-8004?
GroupID=BLG5913
Brilliant Violet 605 hCD8a: https://www.biolegend.com/en-ie/products/brilliant-violet-605-anti-human-cd8a-antibody-7651
PE-Cyanine5.5 hCD4: https://www.thermofisher.com/antibody/product/CD4-Antibody-clone-S3-5-Monoclonal/MHCD0418

\section{Eukaryotic cell lines}

Policy information about cell lines

Cell line source(s)

SK-N-AS, SK-N-FI, and NB-SD neuroblastoma cancer cell lines were obtained from the Maris Lab cell line bank. Other human cancer cell lines, including 293T (human embryonic kidney), Jurkat (acute T cell leukemia), SW620 (Dukes' type C, colorectal adenocarcinoma), HEPG2 (hepatocellular carcinoma), and KATO III (gastric carcinoma) were obtained from American Type Culture Collection (ATCC). Platinum-A (Plat-A) cells were obtained from Cell Biolabs. Primary human T cells were obtained from anonymous donors through the Human Immunology Core at the Perelman School of Medicine at the University of Pennsylvania (Philadelphia, Pennsylvania).

Authentication

Short-tandem repeat profiling of cell lines was provided by ATCC upon purchase, and Maris Lab cell line bank lines were regularly authenticated by short-tandem repeat profiling.

Mycoplasma contamination

Cells were regularly tested for mycoplasma contamination and found to be negative.

Commonly misidentified lines

(See ICLAC register)

No commonly misidentified cell lines were used to the authors' knowledge.

\section{Animals and other organisms}

Policy information about studies involving animals; ARRIVE guidelines recommended for reporting animal research

Laboratory animals

Wild animals

Field-collected samples

Ethics oversight
NSG ( NOD SCIO Gamma) 6-8 week old Females, Jackson Labs Stock number 005557. Mice were kept in a 12 hour light/dark cycle at $20-23^{\circ} \mathrm{C}$ with $30-70 \%$ humidity.

No wild animals were used in this study.

No field-collected samples were used in this study.

All experiments were conducted under a protocol approved by the Children's Hospital of Philadelphia Institutional Review Board, and all in-vivo experiments were conducted under conditions approved by the Institutional Animal Care and Use Committee at the Children's Hospital of Philadelphia. 


\section{Human research participants}

Policy information about studies involving human research participants

Population characteristics

Patient-derived xenografts and high-risk neuroblastoma tumors used for immunopeptidomics and/or in-vivo models were obtained through the ongoing Children's Oncology Group (COG; https://childrensoncologygroup.org/) neuroblastoma biobanking study NCT00904241. All newly diagnosed patients with suspected neuroblastoma, suspected ganglioneuroblastoma, or suspected ganglioneuroma/maturing subtype seen at COG institutions are eligible for this study. Total human primary T cells (CD4/8+) were obtained from the Human Immunology Core at the Perelman School of Medicine at the University of Pennsylvania.

Recruitment

Recruitment for COG biobanking study NCT00904241 was conducted according to study design as indicated on the ClinicalTrials.gov homepage: https://clinicaltrials.gov/show/NCT00904241

Ethics oversight

For human tissue samples, informed consent from each research subject or legal guardian was obtained for each deidentified tumor and blood sample used in this study through the COG neuroblastoma biobanking study NCT00904241

Note that full information on the approval of the study protocol must also be provided in the manuscript.

\section{ChIP-seq}

\section{Data deposition}

$\bigotimes$ Confirm that both raw and final processed data have been deposited in a public database such as GEO.

$\square$ Confirm that you have deposited or provided access to graph files (e.g. BED files) for the called peaks.

Data access links

May remain private before publication.

Files in database submission

Genome browser session (e.g. $\underline{\operatorname{UCSC}})$

For "Initial submission" or "Revised version" documents, provide reviewer access links. For your "Final submission" document, provide a link to the deposited data.

Provide a list of all files available in the database submission.

Provide a link to an anonymized genome browser session for "Initial submission" and "Revised version" documents only, to enable peer review. Write "no longer applicable" for "Final submission" documents.

\section{Methodology}

Replicates

Sequencing depth

Describe the experimental replicates, specifying number, type and replicate agreement.

Antibodies

Describe the sequencing depth for each experiment, providing the total number of reads, uniquely mapped reads, length of reads and whether they were paired-or single-end.

\section{Peak calling parameters number. \\ Data quality \\ Specify the command line program and parameters used for read mapping and peak calling, including the ChIP, control and index files used. \\ Software \\ Describe the methods used to ensure data quality in full detail, including how many peaks are at FDR 5\% and above 5-fold enrichment. \\ Describe the software used to collect and analyze the ChIP-seq data. For custom code that has been deposited into a community repository, provide accession details.}

Describe the antibodies used for the ChIP-seq experiments; as applicable, provide supplier name, catalog number, clone name, and lot

\section{Flow Cytometry}

\section{Plots}

Confirm that:

\The axis labels state the marker and fluorochrome used (e.g. CD4-FITC).

\The axis scales are clearly visible. Include numbers along axes only for bottom left plot of group (a 'group' is an analysis of identical markers).

\All plots are contour plots with outliers or pseudocolor plots.

\A numerical value for number of cells or percentage (with statistics) is provided.

\section{Methodology}

Sample preparation

Cells were harvested and washed once with PBS before resuspension in FACS buffer with appropriate staining reagents or multimers. After 30 minutes of incubation at $4^{\circ} \mathrm{C}$, cells were washed three times with PBS before resuspension in FACS 
Instrument

Software

Cell population abundance

Gating strategy buffer with $1 \%$ formalin fixation. For samples with quantified cell numbers, counting beads were added (CountBright Absolute Counting Beads (Invitrogen, C36950, Lot: 2207530) before sample analysis. Cell viability was determined using LIVE/ DEAD Violet Dead Cell Stain (Invitrogen, L34955, Lot: 2179253, 1:1000).

Beckman Coulter CytoFLEX S (C01161), Beckman Coulter CytoFLEX LX (C40324), BD Biosciences LSR II, Applied Biosystems Attune Acoustic Focusing Cytometer, BD Biosciences FACSJazz Cell Sorter.

Collection was performed using CytExpert (Beckman Coulter) and FACSDiva (v8, BD Biosciences). Flow cytometry analysis was performed using FlowJo v10.7.1 (BD Biosciences).

All sorted populations were often stained and re-analyzed using flow cytometry to ensure phenotypic purity.

All samples were first gated on FSC/SSC lymphocyte populations, single cells (Using SSC-H/SSC-H), and then live cells (negative V450 channel, LIVE/DEAD Violet Dead Cell Stain, Invitrogen). For neuroblastoma cells infected by H1N5 virus, live, single cells were gated by positive staining for FITC virus nucleoprotein (NP) antibody. For TCRs, live, lymphocyte singlets were gated to identify antigen-specific T cells. For transduced CAR T cells, live, single lymphocytes cells were gated using tagged $\mathrm{MHC}$ multimers to identify cells transduced with antigen-specific receptors and to determine cross-reactivity.

$\bigotimes$ Tick this box to confirm that a figure exemplifying the gating strategy is provided in the Supplementary Information. 